\author{
UNIVERSITÀ DEGLI STUDI DELLA TUSCIA \\ FACOLTÀ DI SCIENZE MM. FF. NN. \\ DiPARTIMENTO DI SCIENZE ECOLOGICHE E BIOLOGICHE
}

DOTTORATO DI RICERCA IN EVOLUZIONE BIOLOGICA E BIOCHIMICA XXVI CICLO

Phylogeny and taxonomy of meristematic rock-inhabiting black fungi in the Dothideomycetes based on multi-locus phylogenies

( s.s.d. BIO/02)

\title{
PhD Candidate
}

Eleonora Egidi

Coordinator

Prof. Laura Zucconi Galli Fonseca
Tutor

Prof. Silvano Onofri

Tutor

Dott. Laura Selbmann

Tutor

Prof. Gerrit Sybren de Hoog 
Index

1. INTRODUCTION 3

1.1 THE ROCK SUBSTRATUM $\quad 3$

1.1.1 ROCK MICROBIAL COMUNITIES $\quad 3$

1.1.2 STRATEGIES FOR ROCK COLONIZATION 4

1.2 ROCK INHABITING FUNGI (RIF) $\quad 6$

1.2.1 GENERAL FEATURES 6

1.2.2 DISTRIBUTION OF ROCK FUNGI

1.2.3 ADAPTATIVE STRATEGIES FOR SURVAVAL IN EXTREME CONDITIONS 8

$\begin{array}{ll}1.2 .4 \text { NUTRITION } & 10\end{array}$

1.2.5 CRYOMYCES, A PARADIGMATIC GENUS 11

1.2.6 ORIGINS OF RIF: AN ESTIMATION OF DIVERGENCE TIMES 12

1.2.7 INTEREST OF MELANIZED ROCK FUNGI 13

1.3 METHODS FOR IDENTIFICATION 14

1.3.1 FROM MORPHOLOGY TO MOLECULAR TECNIQUES 14

1.3.2 ITS AS UNIVERSAL DNA BARCODE MARKER FOR FUNGI 15

1.3.3 THE MULTILOCUS APPROACH 16

1.4 PHYLOGENY 16

1.4.1 PHYLOGENY OF FUNGI: WORK IN PROGRESS 16

1.4.2 PHYLOGENETIC STUDIES ON RIF 17

2. AIM OF THE WORK 19

3. MATERIAL AND METHODS 20

$\begin{array}{ll}\text { 3.1 ROCK SAMPLES } & 20\end{array}$

3.1.1 SAMPLING SITES 20

3.2 STRAINS ANALYSED 22

3.3 MORPHOLOGICAL EXAMINATION AND TEMPERATURE DEPENDENT 32 GROWTH STUDY

3.4 DNA EXTRACTION, AMPLIFICATION AND SEQUENCING 32

3.5 PHYLOGENETIC INFERENCE

$\begin{array}{ll}\text { 4. RESULTS } & 37\end{array}$

4.1 THERMAL PREFERENCES

4.2 PHYLOGENETIC INFERENCE 39

5. TAXONOMY $\quad 51$

$\begin{array}{ll}\text { 6. DISCUSSION } & 78\end{array}$

6.1 MOLECULAR DIVERSITY IN RIF

6.2 ECOLOGY OF COLD-LOVING ROCK INHABITING FUNGI 81

7. REFERENCES $\quad 83$

8. ANNEXES 96 


\section{INTRODUCTION}

\subsection{THE ROCK SUBSTRATUM}

\subsubsection{ROCK MICROBIAL COMUNITIES}

Rocks can be defined as any naturally-formed, consolidated material composed of one or more minerals. Minerals are inorganic crystalline solids with a definite chemical composition (Plummer and McGeary, 1996).

Rocky substrates- at the interface between the atmosphere and a solid substrate (lithosphere) - are ancient terrestrial niches. Survival on the rocks represents an exceptional challenge for living systems, as colonizers have to face widely stressful environmental conditions and their settlement, growth and development are influenced by humidity, temperature, deposition of inorganic and organic materials and nutrients and intense solar irradiation. Physical conditions are characterized by wide and constant changes (Gorbushina, 2007) and all these combined stress factors involve an high selective pressure which limits the successful development of cosmopolitan organisms (Sterflinger, 2005).

Specialized microbial communities, called SubAerial Biofilms (SAB) (Gorbushina and Krumbein, 2000) represent a group of ubiquitous, self-sufficient microbial ecosystems which evolved specialization to survive in this extreme ecological niche thanks to a collective growth habit. They represent a crucial and ancient element having a large impact on the evolution of Earth and life on Earth. Sedimentary rock formations have been influenced by the active participation of biofilms throughout the entire geological history of the Earth (Cooke and Whipps, 1993; Krumbein and Lapo, 1996).

SAB are specialized in successful colonization of sediments and rocky surfaces from different hot and cold environments, and at all latitudes where direct contact with the atmosphere and solar radiation occurs (Costerton et al. 1987; Guezennec et al. 1998; Ortega-Morales et al. 2001) Recently, Gorbushina et al. (2000) proposed the idea that "poikilotrophy" and sub-aerial biofilm community are a specific type of strategy developed for survival in harsh environments.

The role of $\mathrm{SAB}$ in rock erosion's process resulted to be essential for soil and vegetation development (Ehlich, 1996; Chertov et al. 2004), as microorganisms promote mineral diagenesis (which can be generally defined as the transformation of a mineral into a different one) and dissolution. An important contribution to the weathering process can be the result of the excretion of 
metabolites, which cause the dissolution of the host-rock through chemical attack on mineral surfaces (Johnstone and Vestal, 1992; Ehrlich, 1998; Gadd and Sayer, 2000). Cyanobacteria and archea are considered the first microbial association of organisms able to colonize terrestrial surfaces, followed by eukaryotic forms (Margulis, 1970; 1982). Algae, fungi and protists, the first eukaryotic organisms conquering terrestrial environment, resulted to be able to resist to stress exposition better than prokaryotes, developing sophisticated survival strategies which led to lichens and plants evolution (McMenamin and McMenamin, 1994).

Nowadays SAB are composed by a complex consortium of bacteria, algae, fungi and lichens, whose composition varies according to nutrients availability and stress factors' fluctuations, even if mechanisms of interaction are still poorly understood (Gorbushina, 2007).

Lithic communities are characterized by polysaccharides extra cellular matrix production (EPS) (Wimpenny, 2000; de los Rios et al. 2002; Kemmling et al. 2004; Omelon et al. 2006), crucial in promoting interactions between organisms, atmosphere and substrate. In particular, EPS (i) facilitate connections among microorganisms, which stimulate bacterial growth and contact with photobionts (Gorbushina et. al. 2005); (ii) change according to atmospheric and climatic modifications; (iii) limit osmotic stress thanks to water retention capability, allowing community survival during scarce water availability periods (Neu, 1996; Potts, 1999; Decho, 2000; Gorbushina, 2007).

\subsubsection{STRATEGIES FOR ROCK COLONIZATION}

Lithic communities can be restricted to rock surface (epilithic) or expand underneath rock crust (endolithic). Endolithic niche is colonized through different strategies by microbial communities; euendolithic organisms (mostly crusty lichens) penetrate the substrate by active perforation, chasmoendolithic microbes take advantage of natural rock fissures, while the so called criptoendolithic communities live in the airspaces or structural cavities in rocks (Golubic et al. 1981; Friedmann et al. 1982). In Fig. 1 a few examples of epilitic, chasmoendolithic and criptoendolithic colonizations are shown. 

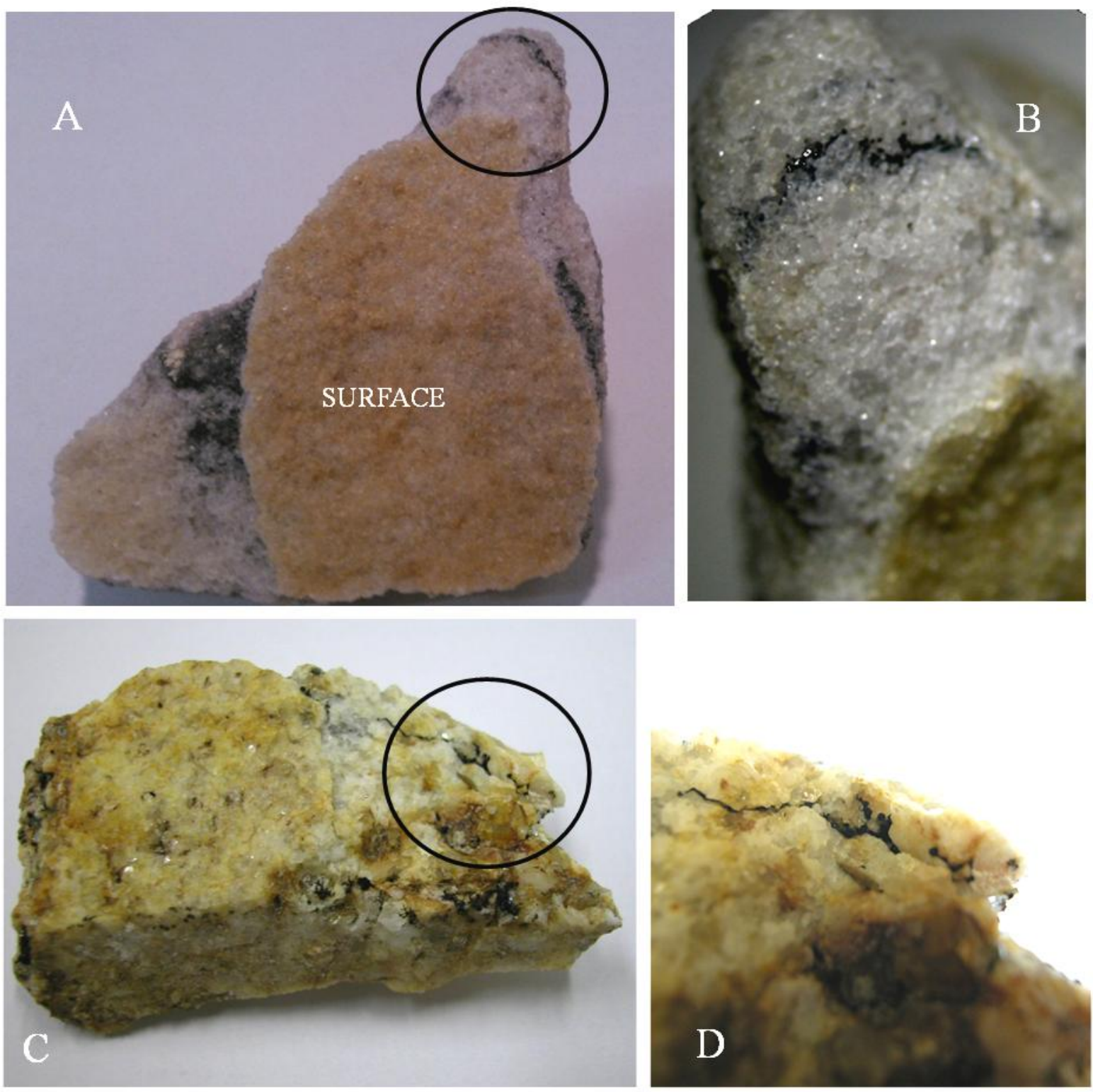

Fig. 1. A. Sandstone with endolithic colonization. B. Detail of endolithic colonization. C, D. Granitoid showing epilithic (lichens) and chasmoendolithic colonization 
Biofilms tendency toward inner rocky substrate colonization can be explained by the necessity of communities to find new and sheltered ecological niches. In fact, during the day and among different seasons, rocks are subject to irregular and wide fluctuations of temperature, humidity and irradiation; temperatures are influenced by solar irradiation and air temperature and, especially in destert areas, can reach extremely low or high temperatures. For example, in Linnaeus Terrace (McMurdo Dry Valleys, Antarctica), rock surfaces have an average winter generally $2{ }^{\circ} \mathrm{C}$ below the outside air temperature, ranging in 1985 from $-47.2{ }^{\circ} \mathrm{C}$ to $-19.4{ }^{\circ} \mathrm{C}$, while summer temperatures on rock surface temperature may reach $20{ }^{\circ} \mathrm{C}$ above that of outside air. (Friedmann et al. 1987). SAB in general, experience high water availability is variable and biofilms run from drought to torrential rains which leave them completely wet. Infrared and UV radiations can be relatively low in the night but extremely high during summer days; in particular high doses of radiations affect mountain sites, deserts and Equator-oriented surfaces. Nutrients availability varies from zero to abundance. In these environments, microorganisms are forced to stress induced dormancy, interrupted by the occasional return of growth-permissive conditions (Gorbushina, 2007).

In order to successfully colonize rock surfaces, SAB employ several physical strategies of penetrating rock, to acquire shelter against extreme environmental influences. They can deepen existing fissures and cracks (Pinna and Salvadori, 1999) or intercrystalline airspaces of rock material (Friedmann, 1982; Urzì and Realini, 1998; Sterflinger et al. 1997). Intracrystalline penetration was also observed (Gorbushina, 2007; Favero-Longo et al. 2011).

Specific features of substrate can widely influence microbes' ability to colonize rocks. Physical characteristics, such as porosity, and chemical composition are crucial to determine SAB growth and survival and can force selective pressure on microbial populations in situ (Gleeson et al. 2005).

\subsection{ROCK INHABITING FUNGI (RIF)}

\subsubsection{GENERAL FEATURES}

RIF are extremophile or extremotollerant organisms showing peculiar common features which allow their survival in oligotrophic environments characterized by extremely high or low temperatures, high UV radiation and osmotic stress combined together. They are usually melanized and organized in microscopic colonies on the rocky substrate, so they are also called black yeasts (Sterflinger, 2005) or microcolonial fungi (MCF) (Staley et al. 1982); because of their ability to form cells' clumps with a peculiar isodiametric, they are also called meristematic fungi (Sterflinger et al. 1999). 
Rock surfaces represent an habitat where only extremotolerant, specialized organisms are able to survive (Gorbushina and Krumbein, 1999; Sterflinger and Krumbein, 1997; Isola et al. 2013b). In this framework, rock-inhabiting fungi (RIF) are efficient lithic substrate colonizers (Staley et al. 1982; Braams, 1992; May et al. 1993; Sterflinger, 2000) and important component of epilithic and endolitic microbial communities. An high degree of specialization and adaptation make RIF predominant organisms in SAB (Gorbushina et al. 1993; Wollenzien et al. 1995). Furthermore, the degree of stress tolerance make fungi the most diverse biota in the Continental Antarctica, one of the most extreme environment on Earth, a continent dominated by microorganisms, where plants are absent (Bridge and Spooner, 2012).

Thanks to complex biomechanical and biochemical processes, they are able to successfully interact with minerals and organic compounds, assuming a crucial role in biogeochemical metabolic process of fixation of nutrients (C, N, P, S) and metals ( essential - $\mathrm{Na}, \mathrm{Mg}, \mathrm{Ca}, \mathrm{Mn}, \mathrm{Fe}, \mathrm{Cu}, \mathrm{Zn}, \mathrm{Co}, \mathrm{Ni}-$ and not essential -Cs, Al, Cd, Hg, Pb), necessary for living organisms survival (Buford et al. 2003).

\subsubsection{DISTRIBUTION OF ROCK FUNGI}

First discovered in hot and cold deserts (Friedmann, 1982, Staley et al. 1982), RIF are now known to be ubiquitous on hard surfaces, in extreme as well as in temperate climates (Urzì et al. 1994; Sterflinger and Prillinger, 2001; Gorbushina, 2007; Gorbushina and Broughton, 2009; Ruibal et al. 2005; 2008; Zucconi et al. 2012). Sampling campaigns conducted in the last fifteen years showed that black fungi are commonly found in both cold and dry climates, from natural rocks to monuments, wherever conditions prevent the settlement of cosmopolitan, fast growing fungi (Gorbushina et al. 2007).

Different studies detected an impressive fungal diversity in Mediterranean zone, even in restricted sampling areas: Ruibal et al. $(2005 ; 2008)$ focused on sampling sites from Spanish Central Mountain System and Mallorca, revealing that only few isolates can be addressed to formally described species from genera Hortaea, Capnobotryella, Catenulostroma, Sarcinomyces, Coniosporium, Capronia, while most fungi belong to new taxa. Fungi from the genera Sarcinomyces and Phaeococcomyces are recurrent in different areas and have been isolated from marble and calcarenite in Greece and Italy (Wollenzien et al. 1997) and from Chersonesos ruins in Crimea; they both were recently included in the genus Knufia (Tsuneda et al. 2011). A number of Capnobotryella species, with unclear phylogenetic positions, have been repeatedly found on Turkish marble monuments (Sert et al. 2007c; Sert and Sterflinger, 2010), while Pseudotaeniolina globosa have been isolated from Santa Maria di Mili church, Messina, Italy (De Leo et al. 2003) and, recently, on statues of the Boyl's Palace in 
Cagliari (Onofri et al. 2012). Species from the genus Exophiala, occasionally found as opportunistic rock colonizers, posses an high rate of stress tolerance but, on the other side, they are also able to react to moderate conditions by faster growth and by morphological changes from meristematic towards mycelial growth (Tesei et al. 2012).

Furthermore, black meristematic fungi are invariably found in cold deserts as well as high mountain tops. Friedmanniomyces, Cryomyces and Elasticomyces, originally isolated from Antarctic rocks, are the largest genera representing this group of highly specialized fungi (Selbmann et al. 2005; 2013; Isola, 2010). Friedmanniomyces is an endemic genus from Victoria Land, Antarctica, while Cryomyces and Elasticomyces, originally isolated from Antarctic rocks, show to have a broader distribution (Selbmann et al. 2005; 2013a). Recently, Saxomyces, a new genus from Alps, has been proposed (Selbmann et al. 2013a). In contrast with rock fungi from temperate environments, these fungi are highly specialized to the cold and are real extremophiles amongst the black fungi.

\subsubsection{RIF ADAPTATIVE STRATEGIES FOR SURVAVAL IN EXTREME CONDITIONS}

Fungi are commonly known to posses skills enabling them to colonizing and persist in novel environments, thanks to a remarkable ecological, biological and morphological plasticity (Agosta and Klemens, 2008).

Rocks are challenging niches;RIF settlement, growing and development on rocky substrates are influenced by many factors, including humidity, UV irradiation, nutrients availability. The lack of competitors caused by the high selective environmental pressure let rock fungi to focus on stress tolerance and successfully colonize prohibitive ecological niches rather than sharping their competition abilities.In particular, cells wall is thick and melanized in order to protect cells against extreme temperatures and desiccation, as well as UV irradiation. Melanins are high molecular weight pigments responsible of the characteristic dark green, brown or black color of RIF, largely contributing to their resistance to chemical and physical stresses. Melanins are negatively charged idrophobic molecules (Langerfelder et al. 2003), often aggregated to proteins and carbohydrates, formed by phenols and indolic compounds polymerization (Butler and Day, 1998). They can be DOPA-melanin (3,4-dihydroxyphenylalanine) or DHN-melanin (1,8-dihydroxynaphtalene) (Butler and Day, 1998). They are synthesized in the cell wall (Diakumaku et al. 1995; Butler and Day, 1998) or released as extracellular polymers in the cultural medium (Kogej et al. 2004). Moreover some melanized fungal species have been found in nuclear reactors and their cooling water systems, suggesting that melanins could confer a remarkable tolerance to ionizing radiation (Zhdanova et al. 
2000), even being responsible of ionizing gamma radiation's conversion into chemical energy by still unknown mechanisms (Dadachova et al. 2007).

Solutes such as trehalose and sucrose, which possess water- retention properties, have been widely detected in endolithic microorganisms, including RIF (Nienow and Friedmann, 1993; WynnWilliams et al. 1999). In particular trehalose is very efficient for its cryoprotective effects during freezing or desiccation (Weinstein et al. 2000) acting as stabilizer of enzyme conformation and phospholipid bi-layers of membranes allowing these surprising organisms to survive complete dehydration (Onofri et al. 2012).

Considering the scare nutrients availability in environments where rock inhabiting fungi typically reside, the high metabolic costs for synthesizing all these compounds significantly affect growth velocity of these fungi which show a very slow growth rate. Meristematic growth (i. e. isodiametric cellular expansion) represents an additional advantage resulting in a minimal surface/volume ratio which allows survival in dry conditions (Wollenzien et al. 1995). The ability to modify cellular polarity (Yoshida et al. 1996), a scarce morphological differentiation and the capacity to rely on airborne sparse nutrients exclusively (oligotrophism) are crucial features for extreme environments inhabiting (Gunde-Cimmerman et al. 2005; Amaral Zettler et al. 2002).

Regulation of metabolic activities is a strategy to balance energy expense according to changes in the composition of the atmosphere and climate. Sterflinger et al. (2012) suggest that in polar environments for large part of the year fungi incur dormancy, a reversible state which stops only when temperature rises and melting water is available, while fungi in hot deserts might profit from dewfall that develops during a short time before sunrise (Ninari and Berliner, 2002). Rock inhabiting fungi are invariably asexual. Indeed, keeping the genetic machinery for recombination active implies too high energetic expense, considering that fungal teleomorphs tend to be elaborate, with fruit bodies and specialized cells in which recombination takes place. Thus, in a framework where all the efforts of the cell are focused on production of few crucial metabolites in order to shelter from environmental stresses, sex represents an energetic cost that a fungus residing in extreme conditions can not afford.

Moreover, in order to decrease energy expense, the life-cycle in these fungi is extremely simplified, usually limited to just a few cells that subdivide and fall apart for passive dispersal. At most, some species exceptionally show some morphological differentiation, with tiny conidiophores as in Recurvomyces mirabilis (Selbmann et al. 2008). The efficiency of RIF dispersal is guaranteed by surprising means to spread. Rock black yeasts, for instance, actively penetrate and brake up the substratum and organize in wind-blown arrays of clump-like cells firmly connected with each other and with rocky substrate by EPS, which can settle elsewhere in order to grow and develop 
successfully. Current biogeographic data suggests that long distance dispersal occurs in rockinhabiting fungi (Gorbushina, 2007; Selbmann et al. 2008; 2013a). However, in few cases, some rock-inhabiting taxa seem to be only present in unique localities or geographical areas (Selbmann et al. 2005).

\subsubsection{NUTRITION}

Nutrition represents a challenge for RIF colonizing cold, oligotrophic environments like desert, Polar or Alpine areas. Oligotrophy is an important adaptation, enabling them to rely only on sparse, airborne nutrients available as dust. Generally black fungi are metabolically scarcely competent producing only few metabolites crucial for survival (Selbmann et al. 2005; Sterflinger, 2006). They are scarcely exigent from a nutritional point of view, being slow growing and able to shift to dormant state even for long periods; they just need simple sugars for growth or easily degradable polymers resulting from photosynthesis of neighboring lichens and algae (Selbmann et al. 2013b), since in desertic and Alpine environments plants are mostly abstent or rare.

Most black yeasts have a very slow growth rate with no significant increases even when cultured on rich media; they are so adapted to the oligotrophic conditions of their natural environments that some also maintain visible mycelial growth, even for short periods, when cultivated on media with no carbon sources.

Recently, Gostinčar et al. (2012) surmised the existence of unconventional pathways of carbon acquisition as additional source of energy alternative to their cohabitation with algae. Atmospheric carbon dioxide fixation could actually exceptionally take place in fungal metabolism, although it lacks a Calvin cycle comparable to that found in plants. It was observed, using ${ }^{14} \mathrm{C}$-labeling, that black fungi isolated from Antarctic cryptoendolithic communities of the McMurdo Dry Valleys, the closest terrestrial analogue for Mars, may actually uptake $\mathrm{CO}_{2}$ (Palmer and Friedman, 1988). The authors' hypotesys is that Antarctic fungi may actually incorporate $\mathrm{CO}_{2}$ by carbossilation of pyruvate (Moses et al. 1959) as it was reported earlier for a number of fungi, although definitive confirmation with a modern-approach planned experiments is still missing (Selbmann et al. 2014).. Such mechanisms could represent a life-sustaining option for slow-growing oligotrophic black fungi in extreme and competitor-free environments. The stunning ability to survive, and even thrive, in environments normally neglected or precluded for most life-form as exposed rock surfaces can be explained by these unusual strategies used by rock fungi in order to gain energy. 


\subsubsection{CRYOMYCES, A PARADIGMATIC GENUS}

A paradigmatic exemple of rock inhabiting fungi's tolerance to environmental stresses is represented by the genus Cryomyces, whose representatives have been isolated from cold Antarctic and Alpine rocks. As recently reviewed by Selbmann et al. (2013c), during last years Cryomyces spp. have been subjected to a number of experiments in order to test stress tolerance. Antarctic strains, which have typical psychrophilic profiles (Van Uden, 1984) with optimal growth temperatures around 10 or at least $15{ }^{\circ} \mathrm{C}$, are unable to grow above $20{ }^{\circ} \mathrm{C}$. In the Antarctic deserts, during summer temperature fluctuactions on rock surfaces can be wide and sudden, acrossing the freezing point over 14 times within 40 minutes causing a repeated freeze-thawing stress to lithobionts; on the opposite side, during winter organisms live in almost permanently frozen conditions. Antarctic black fungi may actually easily tolerate this stress: repeated treatment to $-20+20{ }^{\circ} \mathrm{C}$ did not affect growth ability (Onofri et al. 2007; Onofri et al. 2008). Moreover, strains were proved to tolerate even very high temperatures, since germination ability of Cryomyces spp. is not affected after exposition at $90{ }^{\circ} \mathrm{C}$ for 1 hour (Onofri et al. 2008). Yet, some strains have an eurithermic behavior with optimal growth temperature well above the normal ambient temperature of their natural environment. Cryomyces funiculosus, for instance, a species living in the Alps above $3000 \mathrm{mt}$ of altitudes, has optimal growthat $25{ }^{\circ} \mathrm{C}$ within a range $0-35^{\circ} \mathrm{C}$. It has been surmised that this feature could represent an adaptation to very fluctuating environmental conditions, since in very cold environments exposed rock surfaces may reach temperatures $20{ }^{\circ} \mathrm{C}$ above the air temperatures (Nienow and Friedmann, 1993; Selbmann et al. 2013a).

In cold environments resistance to osmotic stress represent an additional challenge, since water availability decreases when ice crystals form. Moreover, since in some cold environments as the Antarctic desert evaporation may be incredibly high and salt may accumulate conspicuously on rock surface, rock fungi may evolved specific adaptation and may tolerate even considerably high salt concentration. For instance, in Cryomyces spp. visible growth at $\mathrm{NaCl}$ concentration of 25\% (Onofri et al. 2007) is still mainteined, demonstrating a remarkable tolerance to osmotic stresses. Moreover, when testing ability to survive to dissecation with a proteomic approach, Cryomyces antarcticus does not show any response; the organism seems just to down-regulate its metabolism, suggesting that both trehalose and mannitol might play a cell protective role in those fungi (Zacharova et al. 2012) In extreme environments resistance to strong solar radiations is an essential feature since exposition is intense on bare rocks. This is even more necessary in cold deserts as Antarctica or mountain tops, where solar irradiation may be more intense than in hot conditions due the ozone hole insisting particularly at the South Pole or to thin mountain atmosphere. Resistance to radiation has been 
largely documented in Cryomyces. Antarctic strains maintain their ability to germinate after high UV exposition (Onofri et al. 2007) and even space radiations (Onofri et al. 2012) by resisting, rather than repairing potential DNA damages (Selbmann et al. 2011). Moreover, some species of black fungi are strikingly tolerant to ionizing radiation and have been found in both nuclear reactors and their cooling water systems (Zhdanova et al. 2000).

\subsubsection{ORIGINS OF RIF: AN ESTIMATION OF DIVERGENCE TIMES}

Recently, Gueidan et al. (2011) tried to estimate the divergence times of RIF in order to investigate whether they all originated during the same geological period of time and thus infer the ecological conditions under which RIF established.

According to this study, RIF in Chaetothyriales diverged from the lichen order Verrucariales in the middle Triassic, while the origin of RIF in Dothideomycetes dates back to the Devonian and early Carboniferous, suggesting that origin for the rock fungi in Dothideomycetes is previous respect to those in Chaetothyriales. Moreover, the development of chaetothyralean RIF may be related to expansion of arid landmasses in Triassic, leading to extinction and then lack of competition with fast-growing saprobic fungi and, in the same time, an increasing of suitable habitats for RIF, such as bare rock surfaces. On the contrary, global climate of the period of dothideomycetous RIF rising was characterized by cooler temperatures. This result matches with the fact that the cold-endemic genera Cryomyces and Friedmanniomyces, Saxomyces and Elasticomyces belong to the Dothideomyceta lineage only. Nevertheless, authors do not exclude an independent origin of RIF in these two groups.

\subsubsection{INTEREST OF MELANIZED ROCK FUNGI}

The peculiar ability of rock inhabiting fungi to survive in extremely prohibitive environments in the last years attracted the interest of researchers from many different fields of applied biology.

Remarkable high stress tolerance gives new insight in understanding biological mechanisms related to psycrophilic behavior from a molecular point of view, in order to use organisms in industrial processes (Russell, 1998).

Stress resistance features makes them an attractive study object in microbial ecophysiology (Isola $e t$ al. 2011; Nai et al. 2013; Tesei et al. 2012; Zakharova et al. 2012); in particular Cryomyces antarcticus showed to be able to survive in simulated space and Martian conditions (Onofri et al. 2008) as well as out space for 18 months (Onofri et al. 2012). 
Furthermore, RIF have a predominant role in weathering of rocks and biodeterioration of monuments exposed to outdoor conditions (Sert et al. 2007b-c; Sert and Sterflinger, 2010; Marvasi et al. 2012; Isola et al. 2013b). The capability to penetrate and eventually degrade mineral materials is caused by a mechanical action mediated by shrinking and swelling cycles of the colloidal biogenic slimes (EPS) inside the pore system (Warscheid and Braams, 2000) as well as disrupting the structural components of the crystals leading to biopitting and the formation of cracks, fissures, and also exfoliation (Gorbushina et al. 2002; Gorbushina, 2007). In order to have efficient protocols to prevent and control the development of these organisms and set biocontrol programs, a deepen in knowledge of RIF biodiversity, mechanisms of fungi-substratum interaction, and sensitivity to biocides results to be crucial for monuments preservation and restoration (Onofri et al. 2014).

\subsection{METHODS FOR IDENTIFICATION}

\subsubsection{FROM MORPHOLOGY TO MOLECULAR TECNIQUES}

Identification of fungi to species level is a fundamental tasks for mycologist involved in many different research fields, from basic to applied research. For example, reliable species identification of causal agent plays a central role to determine the appropriate treatment of the increasingly frequent fungal diseases in humans (Bialek et al. 2005; Rickerts et al. 2006). Similarly, correct determination of plant pathogens results to be crucial for plant diseases identification and treatment, which might even result in economical implications when involving quarantine measures affecting international trade of plants and plant products (Wingfield et al. 2001; McNeil et al. 2004). Furthermore, all studies related to biodiversity and ecology are fundamentally based on the species unit (alpha diversity) from which the higher levels of biodiversity (i.e. beta and gamma diversity) are derived (Whittaker, 1970).

Taxonomic classification and identification of fungi is traditionally based on classic morphological morphometric criteria, growth parameters, sexual compatibility and/or biochemical features. These parameters can be not enough for a unequivocal identification at species level, as many morphological characters previously thought to be indicative of relatedness have been shown to be homoplasious or otherwise uninformative (Begerow et al. 2007; Lumbsch and Huhndorf, 2007), and convergent morphological evolution as well as plesiomorphic character states appear to be widespread throughout the fungi (Blackwell et al. 2006; Hibbett et al. 2007). In the case of black fungi, a scarce morphological differentiation and the massive production of melanin in the cell wall, which is responsible of the dark pigmentation (black, green, brown), are common traits (Jacobson, 
2000). The scarcity of discriminatory and easily accessible morphological characters makes identification a difficult task, leading to possible classification mistakes if traditional approach is not associated with alternative identification methods.

Thanks to the massive improvements of molecular techniques based on genetic polymorphisms analyses, taxonomic identification of biological specimens based on DNA sequence information (a.k.a. DNA barcoding) is becoming increasingly common in biodiversity science, showing to be highly useful in phylogenetic inference and species delimitation as well.

\subsubsection{ITS AS UNIVERSAL DNA BARCODE MARKER FOR FUNGI}

Fungal kingdom is the second largest eukaryotic kingdom. According to The Dictionary of Fungi (Kirk et al. 2008) 97,330 species of fungi have been described, with additional 1,300 microsporidians, bringing the total to about 99,000 known species. Hawksworth (1991) estimated a comprehensive 1,5 million of species, a number now considered to be conservative due to numerous potential fungal habitats and localities which remain still poorly studied (Hawksworth, 2001). Furthermore, analyses conducted on environmental DNA samples from soil communities revealed a high rate of new species mainly not detectable by classical culture methods, supporting an estimate of 3,5 to 5,1 million species (O'Brien et al. 2005).

In order to improve knowledge and understanding of species diversity in fungal kingdom, most phylogenetic studies have been based on molecular analyses which resulted to be efficient to resolve and increase species detection, revealing cryptic species and huge diversity in plant associated species from Glomeromycota (Wubet et al. 2004) and Sebacinales (Weiß et al. 2004).

Recently, the nuclear ribosomal internal transcribed spacer (ITS) region was designated the official barcode for fungi (Schoch et al. 2012) since it has a long history of use as a molecular marker for species-level identification in ecological and taxonomic studies of fungi (Hibbett et al. 2011).

The use of ribosomal genes for molecular analyses offers different advantages: they are (i) invariably present in all the organisms, (ii) in multiple copies, and (iii) sequences evolution is homogenous according to concerted evolution theory (Feliner and Rosselò, 2007). Despite its universal usage, ITS is a region presenting potential complications as a universal barcode of fungi. Intra-specific as well as intra-individual variability is present (Smith et al. 2007; Simon and Weiß, 2008), and may complicate automated attempts at species identification. Furthermore, multicopy ITS region among species from different taxonomic groups show a not uniform variability (Nilsson et al. 2008). In the same operon, the nuclear ribosomal large (nLSU) and small (nSSU) subunits are present. LSU contains two hypervariable regions, designated D1 and D2, that are flanked by relatively conserved 
sequence regions in most fungi. This arrangement allows LSU gene sequences to be aligned for phylogenetic analysis and represent a popular marker in certain groups, because of their superior species resolution in some taxonomic groups, such as early diverging lineages and ascomycetous yeasts. Ribosomal gene structure is showed in Fig. 2.

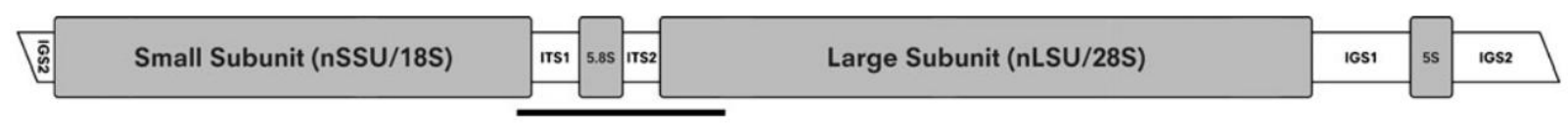

Fig. 2 The nuclear multicopy rDNA repeat of fungi codes for various parts of ribosomal RNA (small subunit -SSU-, 5.8S, large subunit -LSU-, and in some groups $5 \mathrm{~S}$ as well). The tandem repeat includes two-spacer regions, the intergenetic spacer (IGS) and the internal transcribed spacer (ITS). The whole region spanning ITS1, 5.8S and ITS2 can easily be amplified by universal primers in the conserved flanking regions of SSU and LSU and is proposed as a DNA barcode for fungi (black line). From Schoch et al. 2012.

The core issue related to molecular identification methods is defining a threshold value in order to determine when a query sequence is close enough to a reference sequence to be considered conspecific. In publishing practice of new bacterial species, the threshold for intraspecific variability for full-length bacterial SSU sequences is fixed at 3\% (Sneath, 1992). The 3\% threshold value is considered a valid standard for fungi as well: if two ITS sequences differ by less than $3 \%$, they are typically considered conspecific (Hughes et al. 2009). While 3\% cut-off value can be considered valid for some groups of fungi, it results to be not applicable for others (Nilsson et al. 2008). For organisms as Aspergillus and Penicillium (Nilsson et al. 2009) this value is too high, while too low for others (e.g. Cantharellus and Craterellus; Feibelman et al. 1994).

Evidences of differences in ITS intraspecific variability rate within the fungal kingdom suggest caution against simplified approaches to automated ITS-based species delimitation and a "case by case" approach based on the features of taxa into consideration.

\subsubsection{THE MULTILOCUS APPROACH}

Molecular species delineation traditionally was based upon data from a single locus; during the last twenty years many studies suggested that a single gene genealogy does not necessarily represent the organism phylogeny when considering a boarder taxon sampling including distantly related fungi. (Avise and Wollenberg, 1997). 
Because of difficulties on alignment, regions and basal branches are poorly resolved and ITS alone shows to be poorly informative (Moncalvo et al. 2002). Neverthless, the use of simultaneous analysis of different genes (multilocus analysis) to obtain a well-resolved and strongly supported hypothesis for the classification recently demonstrated to be successful for species level identification as well as higher ranking classification.

Taylor et al. (2000) proposed the Genealogical Concordance Phylogenetic Species Recognition (GCPSR), a criterion based on the phylogenetic concordance of multiple unlinked genes, to identify species that cannot be recognized using other criteria. The GCPSR criterion has proved immensely useful in fungi, because it is more finely discriminating than the other criteria in many cases and is currently the most widely used within the fungal kingdom (e.g. Dettman et al. 2003; Fournier et al. 2005; Koufopanou et al. 2001; Le Gac et al. 2007; Pringle et al. 2005).

In a recent study, Schoch et al. (2009) proposed a multilocus approach in order to resolve phylogenetic relationships in the Class Dothideomycetes. Gene targets considered protein coding genes, comprising RNA polymerase II largest and second largest subunits (RPB1 and RPB2) and elongation factor 1 alpha (TEF1), the nuclear small (nSSU) and large subunit (nLSU) ribosomal genes, and the mitochondrial small subunit ribosomal gene (mSSU); RPB2 was identified as the most informative gene, but the importance of ribosomal genes as contributors to resolution of ancient relationships is confirmed.

\subsection{PHYLOGENY}

\subsubsection{PHYLOGENY OF FUNGI: WORK IN PROGRESS}

As previously mentioned, there are roughly 99,000 described species of Fungi, but the actual diversity in the group has been estimated to be comprised between 1,5-5 millions species. Since the first phylogenies using DNA sequence from the nuclear ribosomal genes indicated that fungal groups separated by classical morphological-morphometrical features did not always correspond to phylogenetic clades (Berbee and Taylor, 1992; Spatafora et al. 1995), the molecular approach started becoming a commonly accepted practice in order to resolve fungal phylogenies.

In the last decade, mycologists from many laboratories worldwide joined The Assembling the Fungal Tree of Life (AFTOL), a research consortium dedicated to significantly enhancing our understanding of the evolution of the Kingdom Fungi. Different laboratories developed broad datasets of molecular and non-molecular (e.g., morphological) characters across the Kingdom Fungi. Molecular data sampling focused on six target loci and morphological data emphasized septal pore structure and 
nuclear division, in order to develop specific tools to discover new species and to test explicit hypotheses pertaining to deep nodes of the fungal tree of life (see http://aftol.org/index.php).

Phylogenetic resolution increased considerably with the utilization of slowly evolving protein-coding genes especially suited to the investigation of deep divergences (Liu et al. 1999; Liu and Hall, 2004). The availability of multigene sequence data and numerous genome sequences resulted in several recent phylogenies with comprehensive molecular character sampling across the main Phyla of the Kingdom (James et al. 2006; Kuramae et al. 2006; Spatafora et al. 2006, Schoch et al. 2009). Despite this increase in data, numerous unresolved nodes remain and not all classes and orders have been incorporated into molecular phylogenies (Spatafora et al. 2006). Moreover, due to fragmentary taxon sampling, future species description may remarkably change our knowledges about evolution and phylogenetic relationships within Fungal Kingdom.

\subsubsection{PHYLOGENETIC STUDIES ON RIF}

In this framework, rock inhabiting fungi represent a group of fungi whose phylogenetic relationships are still not completely understood.

Although they have a very limited morphological diversity, the ability of microfungi to grow on rocky substrates revealed to be a polyphyletic trait, assessing RIF in two different classes of Ascomycota, namely Eurotiomycetes (order Chaetothyriales) and Dothideomycetes (mainly the orders Capnodiales, Dothideales and Pleosporales) (Sterflinger et al. 1999; Ruibal 2004; Ruibal et al. 2005, 2008, 2009; Gueidan et al. 2009).

In Eurotiomycetes, multigene phylogenetic analyses have shown that RIF cluster in early diverging lineages of Chaetothyriales, demonstrating that the most recent common ancestor of both lichenised Verrucariales and pathogenrich Chaetothyriales was probably a rock-inhabiting fungus (Gueidan et al. 2008). It was hypothesized that adaptations to life in extreme conditions might have been a prerequisite for the evolution of human pathogenicity (de Hoog, 1993; Haase et al. 1999; Gueidan et al. 2008) and lichenisation in this class (Gueidan et al. 2008).

Ruibal et al. (2009) released an extended multilocus analysis in order to assess the phylogeny of RIF within the Dothideomycetes and they investigated the relationships among RIF, lichen-forming fungi and plant-associated fungi. In this class, only very few human pathogens are known (de Hoog et al. 2000) while associations with plants and in particular plant pathogenicity are very common (Schoch et al. 2006; Crous et al. 2007; 2009). Additionally, lichenised species also appeared to be nested within Dothideomycetes (Lutzoni et al. 2004; James et al. 2006; Del Prado et al. 2006; Muggia et al. 2008; 2013; Nelsen et al. 2009). 
Ruibal's study revealed that several well-supported groups of rock-inhabiting fungi could not be attributed to any known family or order, suggesting that the biodiversity within this class remains severely underestimated. 


\section{AIM OF THE WORK}

Ruibal et al. (2009) produced the first and, so far, only multilocus study focused on RIF phylogenetic affiliations assesment within Dothideomycetes Class, revealing the underestimated biodiversity of this particular group of fungi. Part of these fungi inhabiting cold environments have been described in a series of papers introducing novel genera, such as Cryomyces, Recurvomyces, Saxomyces, and Elasticomyces, including a genus that is endemic to Antarctica, Friedmanniomyces (Onofri et al. 1999; Selbmann et al. 2005; 2008; 2013a), but this is a limited number respect to the available RIF. Indeed, during the last decade rock sampling has been intensified and hundreds of strains have been isolated (Ruibal, 2004; Isola, 2010). Rock samples were collected by mountaineers and skilled excursionists from almost inaccessible locations such as rock formations and nunataks in the Antarctic, including the McMurdo Dry Valleys, and mountain peaks in the Andes, Alps and Indian Himalayan range. The isolates are stored in the Culture Collection of Fungi from Extreme Environments (CCFEE), Italian National Antarctic Museum (Mycological Section), DEB, Università degli Studi della Tuscia Viterbo, Italy. This collection supplements also the strains originating from monuments in the Mediterranean area. Strains isolated by C. Ruibal are stored in CBS collection, in the CBS-KNAW Fungal Biodiversity Centre in Utrecht, The Netherlands. Due to these considerable efforts, the number of RIF isolates available to date is enormous and account to several hundreds. Yet, most of these strains remained nameless, lack a formal species description, and their phylogeny has never been properly assessed.

Aim of this work is to fill this gap emphasizing taxonomic studies into the largely unknown generic diversity of rock-inhabiting fungi affiliated to the Dothideomycetes based on a robust phylogeny for a deep assessment of unnamed RIF isolates available in the reference collections (CCFEE in Viterbo, Italy and the CBS-KNAW Fungal Biodiversity Centre in Utrecht, The Netherlands). The building of an appropriate taxonomic framework for these fungi is also a crucial and necessary step to further investigate ecological associations between RIF and phylogenetically related species, providing a basis for reconstruction of evolutionary pathways and elucidate the extreme adaptations in these extraordinary organisms. 


\section{MATERIAL AND METHODS}

\subsection{ROCK SAMPLES}

\subsubsection{SAMPLING SITES}

Sampling sites spanned different climatic zones in Europe, Asia, South America and Antarctica. Samples from Europe originated mainly from Mediterranean mountains, coastal localities and islands, as well from United Kingdom. Mountain study sites at the isle of Mallorca included localities as Manut II and Cala Sant Vicenç which are pure calcite limestone outcrops of Mesozoic origin. Manut II area is approximately 500 asl (above sea level), in a northern and shady exposure, receiving about $1000 \mathrm{~mm}$ rainfall distributed evenly during the year. The Cala Sant Vicenç site is a southward oriented cliff a few meters asl, sun-exposed and semiarid, affected by sea spray; yearly rainfall is less than $800 \mathrm{~mm}$ (Ruibal et al. 2005).

Four sampling sites, i.e. La Cabrera, Patones, Puebla de La Sierra and Atazar, were located within an area of about $200 \mathrm{~km}^{2}$ in the Iberian Peninsula. From a geo-structural point of view the zone is complex, with pure granite formations of Plutonic origin (La Cabrera) as well as metamorphic, black slate from the Silurian period (Atazar), cretaceous limestones, dolomites (Patones), quartzite and sandstones (Puebla de la Sierra). The area receives about 600-700 $\mathrm{mm}$ rainfall, irregularly distributed over the year. Summers are dry and hot, with temperatures ranging from 5 to $40^{\circ} \mathrm{C}$, while winters are humid and almost continental with temperatures between 15 and $-10{ }^{\circ} \mathrm{C}$ (Ruibal et al. 2008).

Four sampling sites are located in Sardinia, Italy. Boyl Palace and Sant' Elia-Perdusemmini beach are located in the town of Cagliari, while Cala Domestica, close to Buggerru (Carbonia-Iglesias), and Cala di Torre Argentina (Oristano) are two beach sites. Boyl Palace was built in 1840; it is a historical building characterized by a marble balustrade surmounted by statues. Sant' EliaPerdusemmini beach, with its miocenic calcareous cliffs, is located below the homonymous sighting coastal tower. Both sites have a Mediterranean climate; summers are hot, dry with temperatures reaching up to $40{ }^{\circ} \mathrm{C}$ and sometimes with high humidity; winters are mild with temperatures ranging between $5-15^{\circ} \mathrm{C}$ (rarely below zero). The area receives about $400 \mathrm{~mm}$ rainfall per year, mainly concentrated in autumn and winter.

Similar climatic condition with temperatures ranging from -2 to $14{ }^{\circ} \mathrm{C}$ in winter and 25 to $35^{\circ} \mathrm{C}$ in summer, are found in Cala Domestica and Cala di Torre Argentina, two of the most scenic bays of 
western coast of Sardinia enclosed by limestone headlands. The area receives about 500-600 $\mathrm{mm}$ rainfall, with a peak in autumn.

The Vallerano study area, located in central Italy few kilometers to the north of Rome, is all that remained after the collapse of the Benedectine church with early mediaeval wall paintings, dating back to the 10th century. Carved into a tuffaceous high cliff along a small stream at 356 meters asl, the phytoclimatic map of the Latium region affiliates the studied area to the temperate region and belongs to the lower/upper hilly thermotype, upper hyper humid/lower humid ombrotype of the mesaxeric region (Zucconi et al. 2012).

Five sampling sites are located in the Italian Alps: three in Monte Rosa (Punta Indren, Stolenberg, Colle delle Pisse), one in Stelvio National Park (Val de la Mare) and one in the Siusi Alps. All sites are between 2500 and 3200 meters asl where vegetation is very scarce and represented only by cacuminal species. The Italian side of Monte Rosa is southern-exposed and includes about $50 \mathrm{~km}^{2}$ of glaciers, originated from the Penninic nappe in the Paleozoic. Geologically it is characterized by gneiss and mica schist. Stolenberg, Colle delle Pisse and Punta Indren are located in Val de la Lys area, which is characterized by abundant rainfall (about $1200 \mathrm{~mm}$ per year), with temperatures ranging from -8 to $-11^{\circ} \mathrm{C}$ in winter (with peaks of up to $-23^{\circ} \mathrm{C}$ ) and from 1 to $7{ }^{\circ} \mathrm{C}$ in summer, with lower peak at $-7^{\circ} \mathrm{C}$.

Stelvio National Park is one of the oldest Italian national parks located in the central Alps; altitude spans between 650 and 3900 meters asl. In Val del La Mare locality the Cevedale mountain with a peak of 3769 meters asl is composed mainly of schist. Temperatures range from -26 to $+3{ }^{\circ} \mathrm{C}$ in winter and from -4 to $+18{ }^{\circ} \mathrm{C}$ in summer.

Located in the Dolomites, the region Alpe di Siusi represents Europe's largest high plateau at an altitude from 1680 to 2350 meters asl and characterized by dolomite and volcanic rocks originating from the Triassic era.

Worbarrow Bay is a large, broad and shallow bay on the Isle of Purbeck, Dorset, UK. The cliffs are of Cretaceous origin. In particular the rocks spanning from the Claks at the northwest end of the bay to Cow Corner are between 85 and 145 million years old, while the sediments that form the peninsula are Portland limestones, which are dated 150 million years old. The climate in this sampling area is influenced by the Gulf Stream, and annual rainfall is up to $3000 \mathrm{~mm}$, with wet summers mostly below $17^{\circ} \mathrm{C}$; temperatures during winter are between 0 and $7{ }^{\circ} \mathrm{C}$ and occasional snowfall.

Two sampling sites are located in South America. Aconcagua is the highest mountain in the Americas at 6960 meters asl. It is located in the Andes mountain range and gives rise to a number of glaciers. Rocks are of volcanic origin (Maksaev et al. 2009). Winter temperatures are between 0 and 
$-30^{\circ} \mathrm{C}$ and the peak is covered with perennial snow. Rainfall accumulates to $100-200 \mathrm{~mm}$ annually. The other sampling site is located in Patagonia at the foot of mount Fitz Roy (also known as Chaltén) near the waterfall Chorrillo del Salto at about $450 \mathrm{~m}$ asl. The Chaltén peak rises to $3405 \mathrm{~m}$ and is near the eastern edge of the southern Patagonian ice-field. It is located on hard rocks of the Gondwanan Paleozoic basement and indigenous rocks of the Mesozoic and Cenozoic. Summer average temperature is below $18{ }^{\circ} \mathrm{C}$ during the day and below $5^{\circ} \mathrm{C}$ during the night. Winters bring snow in moderate quantities and average temperatures around $3{ }^{\circ} \mathrm{C}$ during day and $-4{ }^{\circ} \mathrm{C}$ at night time, with peaks of $-20^{\circ} \mathrm{C}$.

Two sampling sites are located in the Ladakh region in the Indian Himalayan mountain system, the youngest mountain ranges on the planet consisting mostly of uplifted sedimentary and metamorphic rock. Ladakh is the highest plateau of the province Kashmir, some sections are located above 3000 meters asl. Temperatures range between -15 to $0{ }^{\circ} \mathrm{C}$ in winter and $10-17{ }^{\circ} \mathrm{C}$ in summer.

Rock samples from Antarctica were collected during XIX and XXVI Italian Antarctic expedition from different localities in both Southern Victoria Land (Battleship Promontory, Linnaeus Terrace, and University Valley, last two are located in the McMurdo Dry Valleys, one of the planet's most extreme cold deserts) and in Northern Victoria Land (Inexpressible Island, Widowmaker Pass, Starr Nunatak, Tarn Flat, Vegetation Island), as well as in the Antarctic Peninsula. Details on sampling locations are provided by Selbmann et al. (2005). Starr Nunatak marks the north side of the mouth of Harbord Glacier, while Tarn Flat is a wide flat area between Widowmaker and Reeve glaciers, affected by catabatic winds. Rocks of both sites are Cambro-Ordovician, granite harbour granitoids (Rossetti et al. 2000). Vegetation Island is a narrow island at north of Inexpressible Island and west of the Northern Foothills, along the coast of Victoria Land; rocks of this site are densely covered with lichens. Cliffs are composed of a granito-metamorphic complex (Skinner et al. 1980). The total precipitation in Antarctica, averaged over the entire continent, is about $166 \mathrm{~mm}$ per year, falling as snow (Vaughan et al. 1999), but in the McMurdo Dry Valleys it is less than $100 \mathrm{~mm}$ which mostly sublime or is blown away.

\subsection{STRAINS ANALIZED}

Strains analysed have been supplied by the CBS (Fungal Biodiversity Center) and CCFEE (Culture Collection of Fungi From Extreme Environments) collections.

CCFEE strains were collected thanks to isolation effords conducted during the last ten years by different researches involved in rock inhabiting fungi biodiversity study. Rock collection and fungal isolation in laboratory was performed according to Selbmann et al. $(2005 ; 2008)$ 
Rock-inhabiting strains from the Mediterranean (TRN strains), maintained in the CBS collection, have been collected and isolated by Ruibal et al. $(2005 ; 2008)$, according to the described method. Geographical origins of RIF included in this study and details of previously published strains are listed in Table 1. 


\begin{tabular}{|c|c|c|c|c|c|c|c|c|}
\hline \multirow{2}{*}{ Species } & \multirow{2}{*}{ Collection number } & \multirow{2}{*}{ Family } & \multirow{2}{*}{ Geography } & \multirow{2}{*}{$\begin{array}{l}\text { Additional } \\
\text { information }\end{array}$} & \multicolumn{4}{|c|}{ NCBI } \\
\hline & & & & & ITS & nucLSU & RPB2 & $B T 2$ \\
\hline Arthrocatena tenebrio & CBS $136100=$ CCFEE 5413 & $\begin{array}{l}\text { Teratosphaeriaceae } \\
\text { I, Capnodiales }\end{array}$ & Punta Indren, Monte Rosa, Italy & $\begin{array}{l}\text { Ruibal } \text { et al. } \\
\quad \text { (2009) }\end{array}$ & KF309948 & GU250385 & KF310044 & - \\
\hline Catenulomyces convolus & CBS $118609=$ TRN 9 & Incertae saedis & $\begin{array}{l}\text { La Cabrera, Central Mountain } \\
\text { System, Spain }\end{array}$ & $\begin{array}{l}\text { Ruibal et al. } \\
\quad \text { (2008) }\end{array}$ & AY843045 & - & - & - \\
\hline $\begin{array}{l}\text { Constantinomyces } \\
\text { macerans }\end{array}$ & CBS 119304=TRN440 & $\begin{array}{l}\text { Teratosphaeriaceae } \\
\text { I, Capnodiales }\end{array}$ & $\begin{array}{c}\text { Patones, Central Mountain System, } \\
\text { Spain }\end{array}$ & $\begin{array}{l}\text { Ruibal et al. } \\
\quad \text { (2008) }\end{array}$ & AY843139 & KF310005 & KF310081 & KF546776 \\
\hline Constantinomyces minimus & CBS $118766=$ TRN159 & $\begin{array}{l}\text { Teratosphaeriaceae } \\
\text { II, Capnodiales }\end{array}$ & $\begin{array}{l}\text { La Cabrera, Central Mountain } \\
\text { System, Spain }\end{array}$ & $\begin{array}{l}\text { Ruibal et al. } \\
\quad \text { (2008) }\end{array}$ & AY843066 & KF310003 & KF310077 & KF546771 \\
\hline $\begin{array}{l}\text { Constantinomyces } \\
\text { nebulosus }\end{array}$ & CBS 117941=TRN262 & $\begin{array}{l}\text { Teratosphaeriaceae } \\
\text { I, Capnodiales }\end{array}$ & $\begin{array}{c}\text { Atazar, Central Mountain System, } \\
\text { Spain }\end{array}$ & $\begin{array}{l}\text { Ruibal et al. } \\
\quad \text { (2008) }\end{array}$ & AY843109 & KF310014 & KF310068 & KF546774 \\
\hline Constantinomyces virgultus & CBS $117930=$ TRN79 & $\begin{array}{l}\text { Teratosphaeriaceae } \\
\text { II, Capnodiales }\end{array}$ & Cala San Vincenç, Mallorca, Spain & $\begin{array}{l}\text { Ruibal et al. } \\
(2005,2009)\end{array}$ & AY559339 & GU323964 & KF310082 & KF546781 \\
\hline Cryomyces antarcticus & CBS 116301=CCFEE 534 & Incertae saedis & $\begin{array}{c}\text { Linnaeus Terrace, McMurdo Dry } \\
\text { Valleys, Antarctica }\end{array}$ & $\begin{array}{l}\text { Selbmann et al. } \\
\text { (2005, 2008); } \\
\text { Ruibal } \text { et al. } \\
\quad(2009)\end{array}$ & DQ028269 & - & - & - \\
\hline Cryomyces antarcticus & CCFEE 690 & Incertae saedis & $\begin{array}{c}\text { Linnaeus Terrace, McMurdo Dry } \\
\text { Valleys, Antarctica }\end{array}$ & $\begin{array}{l}\text { Selbmann et al. } \\
\text { (2005, 2008); } \\
\text { Ruibal et al. } \\
\text { (2009) }\end{array}$ & KC315853 & - & - & - \\
\hline Devriesia antarctica & CBS 136103=CCFEE 451 & $\begin{array}{l}\text { Teratosphaeriaceae } \\
\text { II, Capnodiales }\end{array}$ & $\begin{array}{c}\text { Linnaeus Terrace, McMurdo Dry } \\
\text { Valleys, Antarctica }\end{array}$ & $\begin{array}{l}\text { Selbmann et al. } \\
\text { (2005, 2008); } \\
\text { Ruibal et al. } \\
\quad(2009)\end{array}$ & KF309978 & GU250360 & KF310085 & KF546729 \\
\hline
\end{tabular}


Devriesia antarctica

Devriesia antarctica

\section{Devriesia adstricta}

\section{Devriesia bulbillosa}

\section{Devriesia compacta}

Devriesia modesta

\section{Devriesia simplex}

Elasticomyces elasticus

Elasticomyces elasticus

Elasticomyces elasticus

Elasticomyces elasticus

Elasticomyces elasticus

Elasticomyces elasticus
CCFEE 5312

CBS 118292=TRN96

CBS 118285=TRN81

CBS 118294=TRN111

CBS 137182=CCFEE 5672

CBS $13718=$ CCFEE 5681

CCFEE 5474

CCFEE 5490

CCFEE 5505

CCFEE 5506

CCFEE 5525

CCFEE 5526

$$
\text { II, Capnodiales }
$$

Teratosphaeriaceae II, Capnodiales

Teratosphaeriaceae II, Capnodiales

\section{Teratosphaeriaceae} II, Capnodiales

Teratosphaeriaceae II, Capnodiales

Teratosphaeriaceae II, Capnodiales

Teratosphaeriaceae I, Capnodiales

Teratosphaeriaceae I, Capnodiales

Teratosphaeriaceae I, Capnodiales

Teratosphaeriaceae I, Capnodiales

Teratosphaeriaceae I, Capnodiales

Teratosphaeriaceae
Inexpressible Island, SVL,

$$
\text { Antarctica }
$$

Vegetation Island, Antarctica I, Capnodiales

Mallorca, Spain Italy Italy

Aconcagua, Andes, Argentina, America

Aconcagua, Andes, Argentina, America

Antactic Peninsula, Antarctica

Antactic Peninsula, Antarctica
KF309980

KF31002

KF310087

KF546730

KF309979

KF310020

KF310086

Unpublished

Ruibal et al. (2005)

AY559346

KF310022

KF310103

KF546783

Cala San Vincenç, Mallorca, Spain

Ruibal et al. (2005)

AY559341

KF310029

KF310102

Ruibal et al. (2005, 2009)

AY559351 GU323967

KF310095

KF546761

Grotta del Salvatore, Vallerano (VT),

KF309984

KF310026

KF310093

Grotta del Salvatore, Vallerano (VT),

KF309985

KF310027

(2012)

Tarn Flat, NVLNVL, Antarctica

Selbmann et al (2008)

KF309950

KF309991

Unpublished

Tarn Flat, NVLNVL, Antarctica

Ruibal et al. (2009)

KF309951

KF309992

KF310047

KF309952

KF309996

KF310069

KF309953

KF309997

KF310048

KF309998

KF310049
KF309954

KF309955
KF309999
KF546752

\section{KF546744}

KF546746

Unpublished

KF546748

Unpublished 


\begin{tabular}{|c|c|c|c|c|c|c|c|c|}
\hline Elasticomyces elasticus & CCFEE 5537 & $\begin{array}{l}\text { Teratosphaeriaceae } \\
\text { I, Capnodiales }\end{array}$ & $\begin{array}{l}\text { Ladakh, Kanji-la Valley Himalayas, } \\
\text { India }\end{array}$ & & KF309956 & KF310001 & Unpublished & KF546754 \\
\hline Elasticomyces elasticus & CCFEE 5538 & $\begin{array}{l}\text { Teratosphaeriaceae } \\
\text { I, Capnodiales }\end{array}$ & $\begin{array}{l}\text { Ladakh, Kanji-la Valley Himalayas, } \\
\text { India }\end{array}$ & & KF309957 & KF310000 & Unpublished & KF546755 \\
\hline Elasticomyces elasticus & CCFEE 5543 & $\begin{array}{l}\text { Teratosphaeriaceae } \\
\text { I, Capnodiales }\end{array}$ & $\begin{array}{l}\text { Ladakh, Kanji-la Valley Himalayas, } \\
\text { India }\end{array}$ & & KF309958 & KF309993 & KF310051 & KF546756 \\
\hline $\begin{array}{l}\text { Friedmanniomyces } \\
\quad \text { endolithicus }\end{array}$ & CCFEE 5180 & $\begin{array}{l}\text { Teratosphaeriaceae } \\
\text { I, Capnodiales }\end{array}$ & $\begin{array}{l}\text { Battleship Promontory, McMurdo } \\
\text { Dry Valleys, SVL, Antarctica }\end{array}$ & $\begin{array}{l}\text { Selbmann } \text { et al. } \\
(2005,2008)\end{array}$ & JN885544 & GU250367 & - & - \\
\hline $\begin{array}{l}\text { Friedmanniomyces } \\
\quad \text { endolithicus }\end{array}$ & CBS 119427=CCFEE 524 & $\begin{array}{l}\text { Teratosphaeriaceae } \\
\text { I, Capnodiales }\end{array}$ & $\begin{array}{c}\text { Linnaeus Terrace, McMurdo Dry } \\
\text { Valleys, Antarctica }\end{array}$ & $\begin{array}{l}\text { Selbmann } \text { et al. } \\
\quad \text { (2005) }\end{array}$ & JN885541 & GU250364 & KF310054 & KF546732 \\
\hline $\begin{array}{l}\text { Friedmanniomyces } \\
\quad \text { endolithicus }\end{array}$ & CCFEE 5199 & $\begin{array}{l}\text { Teratosphaeriaceae } \\
\text { I, Capnodiales }\end{array}$ & Trio Nunataks, NVLNVL, Antarctica & & JN885547 & KF310007 & KF310093 & \\
\hline $\begin{array}{l}\text { Friedmanniomyces } \\
\text { endolithicus }\end{array}$ & CCFEE 5283 & $\begin{array}{l}\text { Teratosphaeriaceae } \\
\text { I, Capnodiales }\end{array}$ & $\begin{array}{l}\text { Battleship Promontory, McMurdo } \\
\text { Dry Valleys, SVL, Antarctica }\end{array}$ & & KF309960 & KF310006 & KF310053 & - \\
\hline $\begin{array}{l}\text { Friedmanniomyces } \\
\quad \text { endolithicus }\end{array}$ & CCFEE 5328 & $\begin{array}{l}\text { Teratosphaeriaceae } \\
\text { I, Capnodiales }\end{array}$ & $\begin{array}{l}\text { University Valley, McMurdo Dry } \\
\text { Valleys, SVL, Antarctica }\end{array}$ & & KF309961 & JN885563 & KF310055 & KF546733 \\
\hline $\begin{array}{l}\text { Friedmanniomyces } \\
\text { endolithicus }\end{array}$ & CBS $119426=$ CCFEE 670 & $\begin{array}{l}\text { Teratosphaeriaceae } \\
\text { I, Capnodiales }\end{array}$ & $\begin{array}{l}\text { Battleship Promontory, McMurdo } \\
\text { Dry Valleys, SVL, Antarctica }\end{array}$ & $\begin{array}{l}\text { Selbmann et al. } \\
\quad(2005,2008)\end{array}$ & JN885542 & GU250366 & KF310056 & - \\
\hline Friedmanniomices simplex & CBS 116302=CCFEE 5184 & $\begin{array}{l}\text { Teratosphaeriaceae } \\
\text { I, Capnodiales }\end{array}$ & $\begin{array}{l}\text { Battleship Promontory, McMurdo } \\
\text { Dry Valleys, SVL, Antarctica }\end{array}$ & $\begin{array}{l}\text { Selbmann et al. } \\
(2005,2008)\end{array}$ & DQ028271 & - & - & - \\
\hline Hortaea werneckii & CBS 117931=TRN122 & $\begin{array}{l}\text { Teratosphaeriaceae } \\
\text { II, Capnodiales }\end{array}$ & Cala San Vincenç, Mallorca, Spain & $\begin{array}{l}\text { Ruibal et al. } \\
(2005,2009)\end{array}$ & AY559357 & GU323969 & KF310058 & KF546763 \\
\hline Hyphoconis sterilis & CBS $118321=$ TRN287 & $\begin{array}{l}\text { Teratosphaeriaceae } \\
\text { II, Capnodiales }\end{array}$ & $\begin{array}{c}\text { Atazar, Central Mountain System, } \\
\text { Spain }\end{array}$ & $\begin{array}{l}\text { Ruibal et al. } \\
\quad \text { (2008) }\end{array}$ & AY843126 & KF310032 & - & - \\
\hline Incertomyces perditus & CBS 136105=CCFEE 5385 & $\begin{array}{c}\text { Teratosphaeriaceae } \\
\text { I, Capnodiales }\end{array}$ & Stolenberg, Monte Rosa, Italy & & KF309977 & KF310008 & KF310083 & - \\
\hline
\end{tabular}




\begin{tabular}{|c|c|c|c|c|c|c|c|c|}
\hline Incertomyces vagans & CCFEE 5393 & $\begin{array}{l}\text { Teratosphaeriaceae } \\
\text { I, Capnodiales }\end{array}$ & Punta Indren, Monte Rosa, Italy & & KF309964 & KF310009 & KF310057 & KF546734 \\
\hline Lapidomyces hispanicus & CBS $118764=T R N 126$ & $\begin{array}{l}\text { Teratosphaeriaceae } \\
\text { I, Capnodiales }\end{array}$ & Cala San Vincenç, Mallorca, Spain & $\begin{array}{l}\text { Ruibal et } \\
\text { al.(2005) }\end{array}$ & AY559361 & KF310016 & KF310076 & - \\
\hline Lapidomyces hispanicus & CBS $118355=$ TRN500 & $\begin{array}{l}\text { Teratosphaeriaceae } \\
\text { I, Capnodiales }\end{array}$ & $\begin{array}{c}\text { Puebla la Sierra, Central Mountain } \\
\text { System, Spain }\end{array}$ & $\begin{array}{l}\text { Ruibal et al. } \\
\quad \text { (2008) }\end{array}$ & AY843182 & KF310017 & - & KF546778 \\
\hline Meristemomyces frigidus & CBS 136044=CCFEE 5401 & $\begin{array}{l}\text { Teratosphaeriaceae } \\
\text { I, Capnodiales }\end{array}$ & Stolenberg, Monte Rosa, Italy & $\begin{array}{l}\text { Ruibal et al. } \\
\quad \text { (2009) }\end{array}$ & KF309966 & GU250383 & KF310105 & KF546738 \\
\hline Meristemomyces frigidus & CBS 137179=CCFEE 5457 & $\begin{array}{l}\text { Teratosphaeriaceae } \\
\text { I, Capnodiales }\end{array}$ & Stolenberg, Monte Rosa, Italy & $\begin{array}{l}\text { Ruibal et al. } \\
\quad \text { (2009) }\end{array}$ & KF309967 & GU250389 & KF310063 & KF546741 \\
\hline Meristemomyces frigidus & CBS 137181=CCFEE 5507 & $\begin{array}{l}\text { Teratosphaeriaceae } \\
\text { I, Capnodiales }\end{array}$ & Aconcagua, Andes, Argentina & & KF309970 & KF310013 & KF310066 & KF546749 \\
\hline Meristemomyces frigidus & CBS 136109=CCFEE 5508 & $\begin{array}{l}\text { Teratosphaeriaceae } \\
\text { I, Capnodiales }\end{array}$ & Aconcagua, Andes, Argentina & $\begin{array}{l}\text { Ruibal et al. } \\
\text { (2009) }\end{array}$ & KF309971 & GU250401 & KF310067 & KF546750 \\
\hline Monticola elongata & CBS 137180=CCFEE 5492 & $\begin{array}{l}\text { Teratosphaeriaceae } \\
\text { I, Capnodiales }\end{array}$ & Stolenberg, Monte Rosa, Italy & & KF309968 & KF309994 & $-K F 310064$ & - \\
\hline Monticola elongata & CCFEE 5499 & $\begin{array}{l}\text { Teratosphaeriaceae } \\
\text { I, Capnodiales }\end{array}$ & Colle delle Pisse, Monte Rosa, Italy & $\begin{array}{l}\text { Ruibal et al. } \\
\text { (2009) }\end{array}$ & KF309969 & GU250398 & KF310065 & KF546747 \\
\hline Montincola elongata & CBS 136206=CCFEE 5394 & $\begin{array}{l}\text { Teratosphaeriaceae } \\
\text { I, Capnodiales }\end{array}$ & Stolenberg, Monte Rosa, Italy & & KF309965 & KF309995 & KF310062 & KF546735 \\
\hline Oleoguttula mirabilis & CBS 136101=CCFEE 5522 & $\begin{array}{l}\text { Teratosphaeriaceae } \\
\text { I, Capnodiales }\end{array}$ & Antactic Peninsula, Antarctica & & KF309972 & KF310019 & KF310070 & KF546751 \\
\hline Oleoguttula mirabilis & CBS 136102=CCFEE 5523 & $\begin{array}{l}\text { Teratosphaeriaceae } \\
\text { I, Capnodiales }\end{array}$ & Antactic Peninsula, Antarctica & & KF309973 & KF310031 & Unpublished & Unpublished \\
\hline Perusta inaequalis & CBS 118271=TRN272 & Incertae saedis & $\begin{array}{l}\text { Atazar, Central Mountain System, } \\
\text { Spain }\end{array}$ & $\begin{array}{l}\text { Ruibal et al. } \\
\text { (2008) }\end{array}$ & AY843116 & - & - & - \\
\hline
\end{tabular}




\section{Perusta inaequalis}

CBS 118357=TRN275

Incertae saedis

Petrophila incerta

Petrophila incerta

Petrophila incerta

Phaeotheca triangularis

Phaeotheca triangularis

Phaeotheca triangularis

Pseudotaeniolina globosa

Rachicladosporium alpinum

Rachicladosporium antarcticum

Rachicladosporium inconspicuum

\section{Rachicladosporium} inconspicuum

Rachicladosporium mcmurdoi
CBS 118608=TRN139b

Teratosphaeriaceae

II, Capnodiales

Teratosphaeriaceae II, Capnodiales

Teratosphaeriaceae II, Capnodiales

CCFEE 5540

CCFEE 5578

CCFEE 5579

CCFEE 5734

CBS 136040=CCFEE 5395

CCFEE 5527

CBS 136042=CCFEE 5388

CBS 136043=CCFEE 5456

CBS 119432=CCFEE 5211
Incertae saedis

Incertae saedis

Incertae saedis

Teratosphaeriaceae I, Capnodiales

Cladosporiaceae, Capnodiales

Cladosporiaceae, Capnodiales

Cladosporiaceae, Capnodiales

Cladosporiaceae, Capnodiales

Cladosporiaceae, Capnodiales
Atazar, Central Mountain System, Spain

Ruibal et al.

(2008)

Cala San Vincenç, Mallorca, Spain

Cala San Vincenç, Mallorca, Spain

Cala San Vincenç, Mallorca, Spain

Sant'Elia Perdusemini, (CA), Italy

Cala Domestica, Buggerru (CA), Italy

Sant'Elia Perdusemini, (CA), Italy

Boyl Palace, Cagliari, Italy

Siusi Alps, Monte Rosa, Italy

Antactic Peninsula, Antarctica

Colle delle Pisse, Monte Rosa, Italy

Punta Indren, Monte Rosa, Italy

Battleship Promontory, McMurdo Dry Valleys, SVL, Antarctica

Ruibal et al.

$$
\text { (2005) }
$$

Ruibal et al. (2005, 2009)

Ruibal et al. (2005, 2009)

Ruibal et al. (2009)

Ruibal et al. (2009) (2005, 2008); Ruibal et al.
GU323963

\section{KF310101}

KF309943

KF309945

KF309946

KF309976

KF310010

KF310073

KF546758

KF309936

KF310035

KF310037

KF546736

KF309942 KF309990 KF310043

KF546753

KF309939

GU250380

KF310040

KF309937 GU250388

KF310041

Selbmann et al.

KF309938

GU250371

KF310078 


\section{Rachicladosporium}

monterosium

\section{Rachicladosporium} paucitum

\section{Ramimonilia apicalis}

Ramimonilia apicalis

Recurvomyces mirabilis

Recurvomyces mirabilis

Saxomyces alpinus

Saxomyces alpinus

Saxomyces alpinus

Saxomyces alpinus

Saxomyces alpinus

Saxomyces alpinus
CBS $137178=$ CCFEE 5398

CBS 136041=CCFEE 5458

CBS 118327=TRN437

CBS 118322=TRN443

CBS 119434=CCFEE 5264

CCFEE 5475

CCFEE 5462

CCFEE 5466

CCFEE 5469

CCFEE 5470

CCFEE 5477

CCFEE 5491

\section{Cladosporiaceae,}

Capnodiales

Cladosporiaceae, Capnodiales

Incertae saedis

Incertae saedis

Teratosphaeriaceae

I, Capnodiales

Teratosphaeriaceae

I, Capnodiales

Incertae saedis

Incertae saedis

Incertae saedis

Incertae saedis

Incertae saedis

Incertae saedis
Stolenberg, Monte Rosa, Italy

Val de La Mare, Stelvio National Park, Italy

Patones, Central Mountain System, Spain

Patones, Central Mountain System, Spain

Battleship Promontory, SVL, Antarctica

Stolenberg, Monte Rosa, Italy

Stolenberg, Monte Rosa, Italy

Stolenberg, Monte Rosa, Italy

Stolenberg, Monte Rosa, Italy

Val de La Mare, Stelvio National Park, Italy

Colle delle Pisse, Monte Rosa, Italy

Stolenberg, Monte Rosa, Italy
Ruibal et al. (2009)

Ruibal et al. (2009)

Ruibal et al. (2008, 2009)

Ruibal et al. (2008)

Selbmann et al. (2008); Ruibal et al. (2009)

Selbmann et al. (2008)

KF309962

(2008)

Selbmann et al. (2013)

Ruibal et al. (2009); Selbmann et al. (2013)

Selbmann et al. (2013)

Selbmann et al. (2013)

Selbmann et al. (2013)

Selbmann et al. (2013)

AY843135

AY843141

FJ415477

GU250350

KC315860

KC315861

KC315862

KC315863
KF309940 GU250382 KF310039

\section{$\begin{array}{llr}\text { KF309941 KF309988 } & \text { KF310042 }\end{array}$}

GU250372

KF310059

Unpublised

KC315876

\section{KF310060}

KF546737

KF546742

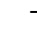

$-$

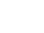


Vermiconia antarctica

Vermiconia antarctica

Vermiconia flagrans

Vermiconia flagrans

Vermiconia flagrans

Vermiconia foris

Undescribed species

Undescribed species

Undescribed species

Undescribed species

Undescribed species
CBS 136107=CCFEE 5488

CBS 118283=TRN124

CBS 118284=TRN104

CBS 118296=TRN114

CBS 136106= CCFEE 5459

CCFEE 502

CCFEE 5569

CCFEE 5575

CBS $136110=$ CCFEE 5764

TRN11
CBS 136108=CCFEE 5489
Teratosphaeriaceae II, Capnodiales

Teratosphaeriaceae II, Capnodiales

Teratosphaeriaceae I, Capnodiales

Teratosphaeriaceae II, Capnodiales

\section{Teratosphaeriaceae} II, Capnodiales

Teratosphaeriaceae II, Capnodiales

Teratosphaeriaceae I, Capnodiales

Teratosphaeriaceae I, Capnodiales

Teratosphaeriaceae I, Capnodiales

Teratosphaeriaceae II, Capnodiales

Incertae saedis
Battleship Promontory, McMurdo Dry Valleys, SVL, Antarctica

Battleship Promontory, McMurdo Dry Valleys, SVL, Antarctica

Mallorca, Spain

Manut II, Mallorca, Spain

Manut II, Mallorca, Spain

Punta Indren, Monte Rosa, Italy

Linnaeus Terrace, McMurdo Dry Valleys, Antarctica

Torre Argentina, Oristano, Italy

Foinaven, Scotland, UK

Worbarrow bay, UK

La Cabrera, Central Mountain System, Spain
KF309982

Unpublished

KF310089

Ruibal et al. (2009)

KF309983

KF310023

KF310090

Ruibal et al.

(2008, 2009)

AY559359

GU323971

KF310097

Ruibal et al. (2005)

AY559348

KF310025

KF310094

Ruibal et al. (2005)

AY559354

KF31002

KF31009

Ruibal et al. (2009)

KF309981

GU250390

KF310088

Selbmann et al. (2005, 2008)

Ruibal et al.

KF309963

GU250363

KF310061 (2009)

KF309974

KF310015

KF310071

KF309975

KF310018

KF310072
KF310028

Ruibal et al. (2008, 2009)
KF309986

AY843046
KF546745

KF546765

KF546760

\section{KF546762}

KF546743 


\begin{tabular}{|c|c|c|c|c|c|c|c|c|}
\hline Undescribed species & CBS 117932=TRN123 & $\begin{array}{l}\text { Teratosphaeriaceae } \\
\text { I, Capnodiales }\end{array}$ & Manut II, Mallorca, Spain & $\begin{array}{l}\text { Ruibal et al. } \\
(2005,2009)\end{array}$ & AY559358 & GU323970 & Unpublished & KF546764 \\
\hline Undescribed species & CBS 118300=TRN137 & $\begin{array}{l}\text { Teratosphaeriaceae } \\
\text { I, Capnodiales }\end{array}$ & Mallorca, Spain & $\begin{array}{l}\text { Ruibal et al. } \\
(2005,2009)\end{array}$ & AY559369 & GU323973 & KF310098 & KF546767 \\
\hline Undescribed species & CBS $118302=$ TRN142 & $\begin{array}{l}\text { Teratosphaeriaceae } \\
\text { II, Capnodiales }\end{array}$ & Cala San Vincenç, Mallorca, Spain & $\begin{array}{l}\text { Ruibal et al. } \\
(2005,2009)\end{array}$ & AY559374 & GU323975 & KF310100 & KF546770 \\
\hline Undescribed species & CBS 117937=TRN211 & $\begin{array}{l}\text { Teratosphaeriaceae } \\
\text { I, Capnodiales }\end{array}$ & $\begin{array}{c}\text { Atazar, Central Mountain System, } \\
\text { Spain }\end{array}$ & $\begin{array}{l}\text { Ruibal et al. } \\
(2008,2009)\end{array}$ & AY843077 & GU323978 & KF310038 & KF546772 \\
\hline Undescribed species & CBS $118768=$ TRN232 & $\begin{array}{l}\text { Teratosphaeriaceae } \\
\text { I, Capnodiales }\end{array}$ & $\begin{array}{c}\text { Atazar, Central Mountain System, } \\
\text { Spain }\end{array}$ & $\begin{array}{l}\text { Ruibal et al. } \\
\quad \text { (2008) }\end{array}$ & AY843091 & KF310011 & KF310079 & KF546773 \\
\hline Undescribed species & CBS $118318=$ TRN258 & Incertae saedis & $\begin{array}{c}\text { Atazar, Central Mountain System, } \\
\text { Spain }\end{array}$ & $\begin{array}{l}\text { Ruibal et al. } \\
\quad \text { (2008) }\end{array}$ & AY843106 & - & 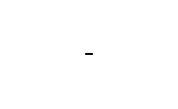 & - \\
\hline Undescribed species & CBS $117950=$ TRN 431 & $\begin{array}{l}\text { Teratosphaeriaceae } \\
\text { I, Capnodiales }\end{array}$ & $\begin{array}{c}\text { Patones, Central Mountain System, } \\
\text { Spain }\end{array}$ & $\begin{array}{l}\text { Ruibal et al. } \\
\quad \text { (2008) }\end{array}$ & AY843129 & KF310004 & KF310080 & KF546775 \\
\hline Undescribed species & CBS $118327=$ TRN437 & $\begin{array}{l}\text { Teratosphaeriaceae } \\
\text { I, Capnodiales }\end{array}$ & $\begin{array}{c}\text { Patones, Central Mountain System, } \\
\text { Spain }\end{array}$ & $\begin{array}{l}\text { Ruibal et al. } \\
(2008,2009)\end{array}$ & AY843135 & GU323984 & - & - \\
\hline
\end{tabular}

Table 1. List of RIF under study. New species in bold with accession numbers of GenBank submissions. Abbreviations used: SVL= Southern Victoria Land; NVL= Northern Victoria Land. 


\subsection{STRAIN CHARACTERIZATION AND MORPHOLOGICAL EXAMINATION}

The dark hyphae isolated from rocks from cold environments where incubated at $15^{\circ} \mathrm{C}$ for 2 weeks, while isolates from monuments grown at room temperature $\left(25^{\circ} \mathrm{C}\right)$ for 2 weeks in malt agar (MA, malt extract 3\%, agar 1.5\%; Delchimica, Napoli, Italy).

Since morphological examination resulted to be inadequate to group isolates and discriminate species, we based identification on comparison of sequences from the whole ITS region (ITS1, ITS2 and $5.8 \mathrm{~S}$ subunit) to recognize numbers of unique genotipes and which isolates were genotipically identical.

Hyphal maturation and conidiogenesis were studied using slide cultures. Inoculation was performed onto $2 \mathrm{~cm}^{2}$ water agar blocks, incubated for 12 weeks at $15{ }^{\circ} \mathrm{C}$ in Petri dishes with wet sterile paper filter to avoid drying out of the medium; agar blocks with inoculated fungi were then transferred onto object slides with lactophenol and observed with light microscopy. Digital images of the samples were acquired using Digital Sight DS-U1 digital camera fitted to a Nikon Eclipse 80i microscope and digitally optimized using Adobe Photoshop 7.0 (Adobe Systems, San Jose, CA, U.S.A.).

Colony descriptions are based on diameter, texture, pigmentation, margin appearance, exudates and colors record after 12 weeks of incubation at $15^{\circ} \mathrm{C}$.

In order to test thermal preferences of isolates, strains were inoculated on Potato-Dextrose Agar (PDA) in culture plates and incubated at 6,15 , and $24{ }^{\circ} \mathrm{C}$. Cultural characteristics were described on PDA at $15{ }^{\circ} \mathrm{C}$. Diameters of colonies were recorded every two weeks. All tests were performed in triplicate.

\subsection{DNA EXTRACTION, AMPLIFICATION AND SEQUENCING}

DNA was extracted from 6-month-old mycelia on MEA slants grown at $10{ }^{\circ} \mathrm{C}$, using the Nucleospin Plant kit (Macherey-Nagel, Germany) and UltraClean® Microbial DNA Isolation kit (Mobio Laboratories, Carlsbad, CA, U.S.A.) following a protocol optimized for fungi. In order to study generic phylogenetic affiliations of RIF, four gene loci were amplified: the large subunit of the nuclear ribosomal RNA operon (nucLSU), the partial second largest subunit of the RNA polymerase II (RPB2), partial $\beta$-tubulin (BT2), and the entire rDNA internal transcribed spacer region (ITS). Primers used are listed in Table 2. 


\begin{tabular}{cccc}
\hline Locus & Primer & Orientation & Reference \\
\hline$B T 2$ & T1 & Forward & O'Donnell and Cigelnik 1997 \\
$B T 2$ & B-Sandy-R & Reverse & Stukenbrock et al. 2012 \\
$B T 2$ & T22 & Reverse & O'Donnell and Cigelnik, 1997 \\
$R P B 2$ & fRPB2-5F & Forward & Liu et al. 1999 \\
$R P B 2$ & fRPB2-414R & Reverse & Quaedvlieg et al. 2011 \\
nucLSU & LSU1Fd & Forward & Crous et al. 2009 \\
nucLSU & LR5 & Reverse & Vilgalys and Hester, 1990 \\
ITS & ITS5 & Forward & White et al. 1990 \\
ITS & ITS4 & Reverse & White et al. 1990 \\
\hline
\end{tabular}

Table 2. List of primers a used for amplification of gene targets.

PCR reactions were performed adding 10 to $40 \mathrm{ng}$ of template DNA to 1.25 units of GoTaq ${ }^{\circledR}$ Flexi DNA Polymerase (Promega, U.S.A.), $0.2 \mathrm{mM}$ of dNTPs, $1.0 \mathrm{mM}$ of $\mathrm{MgCl} 2,5 \mathrm{pmol}$ of each primer and $2.5 \mu \mathrm{l}$ of GoTaq ${ }^{\circledR}$ Flexi Buffer, resulting in a final $12.5 \mu 1$ reaction mixture. Primers are listed in Table 2. PCR amplification conditions were set as follows: an initial denaturation temperature of 96 ${ }^{\circ} \mathrm{C}$ for $3 \mathrm{~min}$, followed by 35 cycles of denaturation temperature of $96{ }^{\circ} \mathrm{C}$ for $45 \mathrm{~s}$, primer annealing at the temperature stipulated in Table 4, primer extension at $72{ }^{\circ} \mathrm{C}$ for $90 \mathrm{~s}$ and a final extension step at $72{ }^{\circ} \mathrm{C}$ for $6 \mathrm{~min}$. In order to obtain a better amplification for the single-copy gene $B T 2$, a touch down PCR was set according to the following conditions: an initial denaturation temperature of 96 ${ }^{\circ} \mathrm{C}$ for $3 \mathrm{~min}$, followed by 10 cycles at $65^{\circ} \mathrm{C}$, decreasing of $1^{\circ} \mathrm{C}$ a in each cycle, 25 cycles of denaturation temperature of $96^{\circ} \mathrm{C}$ for $45 \mathrm{~s}$, primer annealing at the temperature stipulated in Table 3 , primer extension at $72{ }^{\circ} \mathrm{C}$ for $90 \mathrm{~s}$ and a final extension step at $72{ }^{\circ} \mathrm{C}$ for $6 \mathrm{~min}$.

\begin{tabular}{cccc}
\hline Locus & Primer coulples & $\begin{array}{c}\text { Annealing } \\
\text { temperature }\left({ }^{\circ} \mathbf{C}\right)\end{array}$ & $\begin{array}{c}\text { Annealing time } \\
\text { (seconds) }\end{array}$ \\
\hline \multirow{2}{*}{$B T 2$} & T1/3-Sandy-R & 52 & 30 \\
RPB2 & T1/T22 & 52 & 60 \\
nucLSU & fRPB2-5F/fRPB2-414R & 49 & 45 \\
ITS & LSU1Fd/LR5 & 52 & 45 \\
\hline
\end{tabular}

Table 3. Annealing temperatures and duration used for amplification of gene targets. 
The amplification was carried out using MyCycler ${ }^{\mathrm{TM}}$ Thermal Cycler (Bio-Rad, Germany). The amplification products were checked for appropriate size by electrophoresis in agarose gels $1.5 \%$ (IBI Shelton Scientific Inc., Shelton, Conn. USA) in a Owl ${ }^{\mathrm{TM}}$ EasyCast $^{\mathrm{TM}}$ B2 Mini Gel electrophoresis apparatus at 100V (OWL Separation Systems, Thermo Scientific, USA).

Sequencing was carried out using Big Dye Terminator Cycle sequencing kits (Lifetechnologies, U.S.A.) on an ABI 3730xl DNA Analyzers (Lifetechnologies, U.S.A.). Sequences were generated bidirectionally, sequences checked using ChromasPro 1.32 (Conor McCarthy School of Health Science, Griffith University, Southport, Queensland, Australia). and subsequent alignments were generated using MAFFT version 6 (Katoh and Toh, 2010), and manually corrected for sequencing artifacts when necessary. Sequence data were deposited in GenBank (Table 1).

\subsection{PHYLOGENETIC INFERENCE}

A reference phylogeny was inferred from 142 nucLSU sequences obtained from selected RIF strains, and subsequently aligned with comparable sequence data of selected taxa representing the diversity of the class Dothideomycetes, in order to determine the higher hierarchical affiliations of RIF to well-established genera. The nucLSU data matrix comprised selected taxa from recent studies by Schoch et al. (2009), Frank et al. (2010), and Crous et al. (2009). Aligned sequences were taken from TreeBASE (http://treebase.org/treebase-web/home.html/, Study Accessions $\mathrm{N}^{\circ} \mathrm{S}$ S10245, S10547, and S10309, respectively). Newly generated nucLSU sequences of rock inhabiting fungi were added and aligned, resulting in a final alignment comprising 698 taxa.

In order to define generic and species level phylogenetic affiliations of RIF in Capnodiales, different sets of data matrices were assembled. Representative taxa of the main families were sampled and a selection based on the closest taxa according to the nucLSU tree and ITS similarity index using a BLAST search (Altschul et al. 1990), were compiled in the data matrix respectively. To determine species level relationships within the family Teratosphaeriaceae I, a set composed of 63 taxa (including 39 rock-inhabiting strains) was assembled and DNA sequences of nucLSU, RPB2, ITS and BT2 were included in the analysis. Four strains belonging to family Capnodiaceae were chosen as outgroup (Capnodium coffeae CBS 147.52, Microxyphium aciculiforme CBS 892.73, Leptoxyphium fumago CBS 123.26, Polychaeton citri CBS 116435). A separate ITS tree including Elasticomyces elasticus, Friedmanniomyces endoliticus and Friedmanniomyces simplex species representatives was assembled to determine intraspecific molecular diversity rate in these two well established genera and to define a distance standard for molecular species delimitation. 
An alignment based on nucLSU and RPB2 sequences was assembled in order to determine generic affiliations of rock-inhabiting fungi. The data set included a selection of strains belonging to Mycosphaerellaceae, Dissoconiaceae, and Cladosporiaceae, as well as sequences retrieved from GenBank with high sequence similarity to the query (RIF). Capnodium coffeae CBS 147.52, Microxyphium aciculiforme CBS 892.73, Leptoxyphium fumago CBS 123.26, Polychaeton citri CBS 116435 were selected for rooting the tree. Assessment of phylogenetic relationships at species level of strains belonging to Teratosphaeriaceae II was performed assembling a separate set of data matrix based on results obtained from nucLSU and RPB2 analysis, where GenBank sequences of fungi close related to RIF were added. To determine species level relationships within the family Cladosporiaceae, two separate alignments, based on single (ITS) and multilocus (nucLSU, RPB2, ITS and BT2) analysis respectively, were constructed. The ITS data matrix comprised RIF which demonstrated to have high sequence similarity to Cladosporiaceae by a preliminary BLAST search in GenBank. The data set was compiled of ITS sequences of Cladosporium and associated genera identified in a recent study by Bensch et al. (2012) (alignment available in TreeBASE, Study Accession No. S12808), including GenBank sequences of fungi returned by BLAST with high sequence similarity to RIF, and ITS sequences of Hyphoconis sterilis CBS 118321 and Arthrocatena tenebrio CCFEE 5413, here used as outgroup for rooting the tree. The four-gene alignment comprised a selection of sequences chosen according to the ITS analysis; Capnodium coffeae CBS 147.52 and Polychaeton citri CBS 116435 were used for rooting the tree.

A separate ITS alignment was assembled to determine species level affiliations of a specific set of RIF which, on the basis of the first general tree based on nucLSU, was distant from all other genera included, forming a separate cluster within Dothideomycetidae. The data matrix included RIF related to the halophilic fungus Phaeotheca triangularis CBS 471.90, seven strains belonging to the genus Saxomyces and a selection of representative strains of the order Dothideales. The tree was rooted using sequence data retrieved from two Cryomyces antarcticus strains respectively.

For a number of specimens, it was impossible (due to multiple PCR attempts, sequencing failure etc.) to generate sequences for all selected loci and for others congruent sequences were not available in GenBank. Alignment gaps were treated as missing data. The final phylogenetic analyses were performed with the software suite MrBayes (Huelsenbeck and Ronquist, 2001) available at the CIPRES Science Gateway v. 3.1 (http://www.phylo.org/sub_sections/portal/) (Stamatakis et al. 2008). Models of molecular sequence evolution were estimated by FindModel (Posada and Crandall 1998) using the Akaike Information Criterion (AIC). Critical value for the topological convergence diagnostic was set to 0.02 . Two concurrent analyses of four chains were run and a random starting tree was calculated every 1000 Markov Chains Montecarlo generations. Setting details about 
substitution models and number of generations are listed in Table 4. Alternative RAxML trees were performed using RAxML on the Cipres Web Portal. The ML search followed a GTRMIX model of molecular evolution. Support values were obtained with bootstrap analyses of 1,000 pseudoreplicates and final topology is shown in Annexes section (Figures A, B, C). All phylogenetic trees are visualized in TreeView (Page, 1996).

\begin{tabular}{|c|c|c|c|}
\hline Tree & Locus & Model & $\begin{array}{l}\text { Number of } \\
\text { generations }\end{array}$ \\
\hline \multirow{4}{*}{ FIG 4} & RPB2 & TrN: Tamura-Nei plus Gamma TrN & \multirow{4}{*}{2000000} \\
\hline & nucLSU & TrN: Tamura-Nei plus Gamma TrN & \\
\hline & ITS & GTR: General Time Reversible plus Gamma & \\
\hline & BT2 & SYM: Symmetrical Model plus Gamma & \\
\hline FIG 5 & ITS & SYM: Symmetrical Model plus Gamma & 1000000 \\
\hline \multirow{2}{*}{ FIG 6} & RPB2 & TrN: Tamura-Nei plus Gamma TrN & \multirow{2}{*}{1000000} \\
\hline & nucLSU & TrN: Tamura-Nei plus Gamma TrN & \\
\hline \multirow{4}{*}{ FIG 7} & RPB2 & TrN: Tamura-Nei plus Gamma TrN & \multirow{4}{*}{2000000} \\
\hline & nucLSU & TrN: Tamura-Nei plus Gamma TrN & \\
\hline & ITS & GTR: General Time Reversible plus Gamma & \\
\hline & BT2 & SYM: Symmetrical Model plus Gamma & \\
\hline FIG 8 & ITS & SYM: Symmetrical Model plus Gamma & 1000000 \\
\hline \multirow{4}{*}{ FIG9 } & RPB2 & TrNEF: Equal-frequency Tamura-Nei plus Gamma & \multirow{4}{*}{2000000} \\
\hline & nucLSU & TrN: Tamura-Nei plus Gamma TrN & \\
\hline & ITS & SYM: Symmetrical Model plus Gamma & \\
\hline & BT2 & SYM: Symmetrical Model plus Gamma & \\
\hline FIG 10 & ITS & SYM: Symmetrical Model plus Gamma & 1000000 \\
\hline
\end{tabular}

Table 4. Substitution models used in phylogenetic analyses, per locus and tree, and number of generations. 


\section{RESULTS}

\subsection{THERMAL PREFERENCES}

Records of temperature relative to diameters of colonies are listed in Table 5. All strains tested showed optimal growth at $15{ }^{\circ} \mathrm{C}$ with a reduction or absence of growth at $24{ }^{\circ} \mathrm{C}$ indicating a psychrophylic behaviour. Exceptions were Ramimonilia apicalis CBS 118327, Arthrocatena tenebrio CBS 136100 and Constantinomyces virgultus CBS 117930 which, growing better at $24{ }^{\circ} \mathrm{C}$, may be defined as mesophilic. Some of the psychrophilic strains, Arthrocatena tenebrio CBS 136100, Catenulomyces convolutus CBS 118609, Constantinomyces nebulosus CBS 117941, Constantinomyces virgultus CBS 117930, Devriesia adstricta CBS 118292, Perusta inaequalis CBS 118271, Rachicladosporium alpinum CBS 136040, Rachicladosporium inconspicuum CBS 136043, Rachicladosporium paucitum CBS 136041, Ramimonilia apicalis CBS 118327, Vermiconia antarctica CBS 136107 and Vermiconia foris CBS 136106 were able to grow both at $24{ }^{\circ} \mathrm{C}$ and $6{ }^{\circ} \mathrm{C}$; hence they were classified as eurothermic. 


\begin{tabular}{|c|c|c|c|c|}
\hline \multirow[t]{2}{*}{ Species } & \multirow[t]{2}{*}{ Collection number } & \multicolumn{3}{|c|}{ Thermal preferences } \\
\hline & & $6^{\circ} \mathrm{C}$ & $15^{\circ} \mathrm{C}$ & $24^{\circ} \mathrm{C}$ \\
\hline Arthrocatena tenebrio & CBS 136100 & $0.5 \pm 0.2$ & $1.5 \pm 0.5$ & $2.5 \pm 0.2$ \\
\hline Catenulomyces convolutus & CBS 118609 & $2.3 \pm 0.2$ & $5 \pm 0.1$ & $3.8 \pm 0.5$ \\
\hline Constantinomyces macerans & CBS 119304 & $1.0 \pm 0.1$ & $5.2 \pm 0.4$ & - \\
\hline Constantinomyces minimus & CBS118766 & $1.3 \pm 0.1$ & $3 \pm 0.4$ & - \\
\hline Constantinomyces nebulosus & CBS 117941 & $1.5 \pm 0.5$ & $3 \pm 0.8$ & $2.5 \pm 0.1$ \\
\hline Constantinomyces virgultus & CBS 117930 & $1.5 \pm 0.2$ & $1.3 \pm 0.2$ & $3 \pm 0.1$ \\
\hline Devriesia antarctica & CCFEE 5312 & - & 0.8 & - \\
\hline Devriesia adstricta & CBS 118292 & $2 \pm 0.1$ & $2 \pm 0.1$ & $1.5 \pm 0.5$ \\
\hline Devriesia bulbillosae & CBS 118285 & - & $2 \pm 0.1$ & - \\
\hline Devriesia compacta & CBS118294 & - & $\mathbf{1} \pm 0.1$ & - \\
\hline Devriesia modesta & CCFEE 5672 & $0.5 \pm 0.1$ & $2.3 \pm 0.3$ & - \\
\hline Devriesia simplex & CCFEE 5681 & - & $0.8 \pm 0.1$ & - \\
\hline Hyphoconis sterilis & CBS 118321 & $1.1 \pm 0.1$ & $3.5 \pm 0.3$ & - \\
\hline Incertomyces perditus & CBS 136105 & $0.5 \pm 0.1$ & $1.1 \pm 0.2$ & - \\
\hline Incertomyces vagans & CCFEE 5393 & - & $\mathbf{1} \pm \mathbf{0 . 3}$ & - \\
\hline Lapidomyces hispanicus & CBS 118355 & $2.5 \pm 0.2$ & $4 \pm 0.3$ & - \\
\hline Meristemomyces frigidus & CBS 136109 & $1.2 \pm 0.3$ & $3.9 \pm 0.3$ & - \\
\hline Monticola elongata & CBS 136206 & $1.5 \pm 0.3$ & $1.6 \pm 0.2$ & - \\
\hline Oleoguttula mirabilis & CBS 136102 & $1.2 \pm 0.2$ & $\mathbf{1} \pm \mathbf{0 . 5}$ & - \\
\hline Perusta inaequalis & CBS 118271 & $3 \pm 0.2$ & $8 \pm 0.6$ & $7 \pm 0.5$ \\
\hline Petrophila incerta & CBS 118608 & $0.5 \pm 0.1$ & $1.0 \pm 0.3$ & - \\
\hline Rachicladosporium alpinum & CBS 136040 & $1.4 \pm 0.1$ & $3.9 \pm 0.1$ & $2.3 \pm 0.2$ \\
\hline Rachicladosporium antarcticum & CCFEE 5527 & $1.3 \pm 0.2$ & $3.1 \pm 0.1$ & - \\
\hline Rachicladosporium inconspicuum & CBS 146043 & $1.7 \pm 0.1$ & $4.4 \pm 0.1$ & $1.6 \pm 0.2$ \\
\hline Rachicladosporium mcmurdoi & CBS 119432 & - & $2.2 \pm 0.1$ & - \\
\hline Rachicladosporium monterosium & CCFEE 5398 & $1 \pm 0.1$ & $1.6 \pm 0.1$ & - \\
\hline Rachicladosporium paucitum & CBS 136041 & $1.7 \pm 0.3$ & $4.5 \pm 0.1$ & $1.5 \pm 0.3$ \\
\hline Ramimonilia apicalis & CBS 118327 & $3.5 \pm 0.1$ & $4 \pm 0.1$ & $4.5 \pm 0.2$ \\
\hline Vermiconia antarctica & CBS 136107 & $0.4 \pm 0.1$ & $0.7 \pm 0.1$ & $0.4 \pm 0.2$ \\
\hline Vermiconia flagrans & CBS 118296 & $0.5 \pm 0.2$ & $\mathbf{1} \pm 0.3$ & - \\
\hline Vermiconia foris & CBS 136106 & $1.5 \pm 0.1$ & $3.5 \pm 0.3$ & $1 \pm 0.1$ \\
\hline
\end{tabular}

Table 5. Thermal preferences reported as diameters of colonies (in $\mathrm{cm}$ ) after two months of incubation. The values represent the average of three different tests; in bold optimum temperature of growth for each strain. Plates were inoculated on PDA; - no growth. 


\subsection{PHYLOGENETIC INFERENCE}

Sequences of most strains proved to be reproducible (bidirectional sequencing), with the exception of $B T 2$ and $R P B 2$ of some RIF strains related to Teratosphaeriaceae I and II. Difficulties were encountered both in amplification and sequencing steps. Success of amplification was $80 \%$ and 85 $\%$ for $B T 2$ and $R P B 2$ respectively. Often the $B T 2$ fragment could not be properly amplified and, even more frequently, for $R P B 2$ fragments the PCR reaction required several technical repetitions to obtain suitable PCR amplicons.

A general nucLSU tree was reconstructed, based on 799 positions including 698 isolates (Fig. 3), comprising a clade of hitherto undescribed RIF belonging to the order Arthoniales (Ruibal et al. 2009) used as outgroup for tree rooting. Topology of the tree generally was in accordance with the most recent phylogenetic analyses of the Dothideomycetes (Schoch et al. 2006; 2009,; Ruibal et al. 2009). The obtained results show that the family of the Teratosphaeriaceae is indeed polyphyletic (Fig. 3), as it was previously established by Ruibal et al. (2009). The first clade (Teratosphaeriaceae I) includes the representative species of Teratosphaeria as well as rock-inhabiting fungi, namely the genera Friedmanniomyces, Elasticomyces and Recurvomyces (Fig. 4). In our analysis, 44 RIF strains assembled in this clade, which to date contains 67 described species. A second clade, here referred to as Teratosphaeriaceae II (Fig. 6, 7) comprised 22 unidentified rock-inhabiting fungi, clustering with 14 known species occurring on plants, such as Staninwardia suttonii isolated from Eucalyptus robusta, and Pseudoramichloridium henryi found on leaves of Corymbia henryi (Crous et al. 2009), and Pseudoramichloridium brasilianum, isolated from soil. Two RIF strains, CBS 118326 and CBS 118290, isolated from rocks originating from the Patones sampling locality (Spain) and Mallorcan limestone formations, are placed in the family Capnodiaceae (comprising a number of economically significant plant pests); the branch is collapsed in Fig. 3.

In the Dothideomycetidae (Fig.3) two separate clades could be distinguished. One of these contained the species Phaeotheca triangularis (Zalar et al. 1999), whereas in a second cluster no close match with any described fungus could be found except for the Alpine rockinhabiting genus Saxomyces (Selbmann et al. 2013a). In Fig. 10 a more detailed ITS tree is shown; clades are phylogenetically distant from each other such that variable genes are not properly alignable with confidence. Clades 1 and 2 represent undescribed species; the antarctic and alpine RIF genera Cryomyces and Saxomyces were resolved in a basal branch. Phaeotheca triangularis is a halophilic fungus (Zalar et al. 1999), isolated for the first time from an air conditioning system and later found on seawater-impacted rocks. The genera Saxomyces and Cryomyces were shown to belong to the subclass Dothideomycetidae, but could not clearly be attributed to any order or family. 


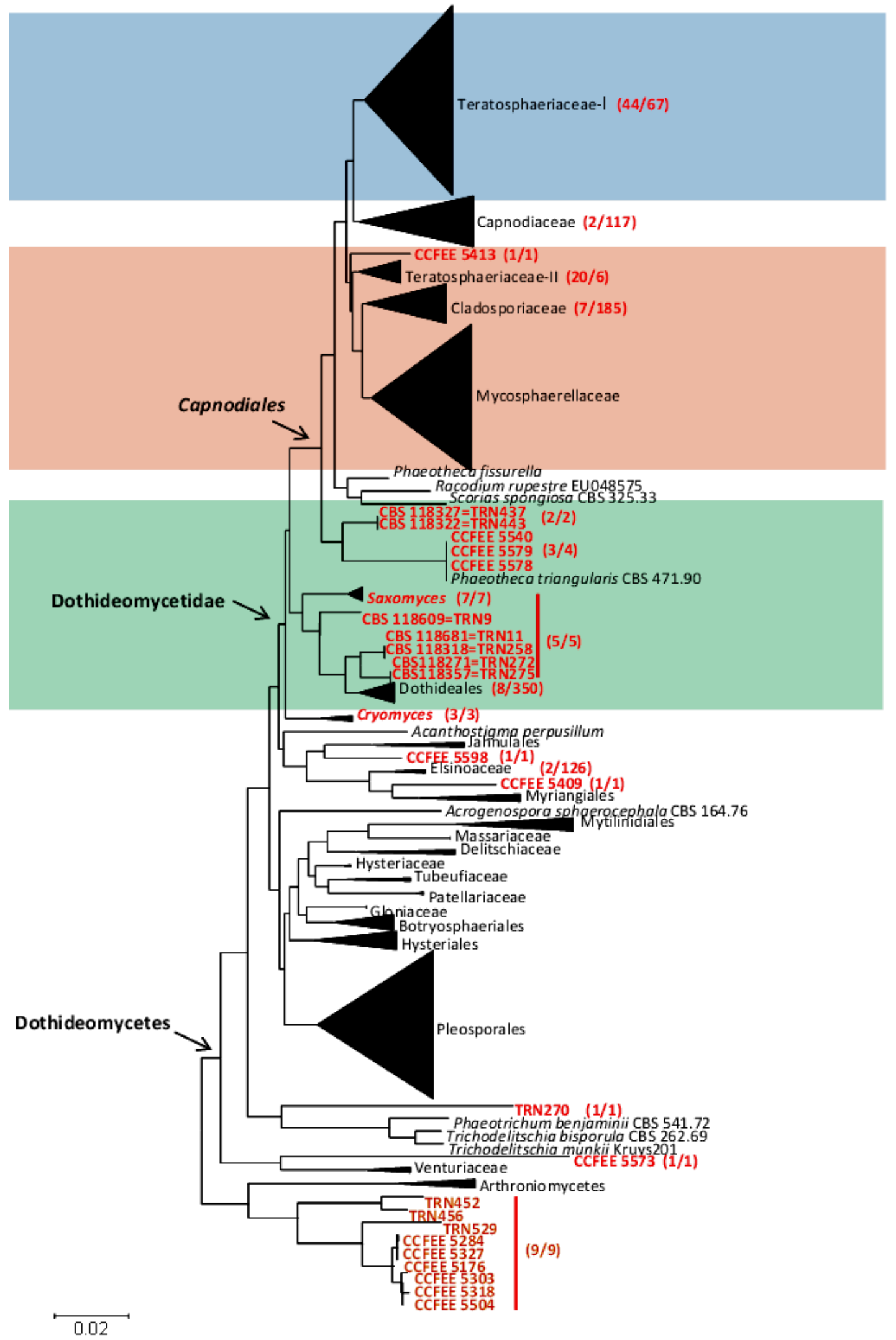

Fig. 1 LSU phylogenetic tree of class Dothideomycetes, resulting from Bayesian analysis of 698 species. Branches are collapsed at ordinal and family levels. Clades are indicated with colored blocks; strains isolated from rocks are indicated with red text; RIF strains used as outgroup are indicated in brown. Numbers between brackets indicate ratio between number of strains isolated from rocks and number of species described in the family according to Dictionary of the Fungi (Kirk et al. 2008). 
A large share of RIF clustered in the order Capnodiales. Combined nucLSU, RPB2, BT2 and ITS data were used to establish species boundaries of RIF within the family Teratosphaeriaceae I (Fig. 4). The alignment had a total length of 1842 bp comprising 61 taxa; in general the ITS, BT2 and RPB2 sequences were considerably variable among the selected taxa (Fig. 4). The ITS and intronic regions of $R P B 2$ and $B T 2$ showed a high degree of polymorphisms, which often were not alignable with sufficient confidence when considering phylogenetically distant strains, and for this reason ambiguous regions were removed from the alignment. Rates of variability of the selected target genes still allowed resolution at species level. The multilocus analysis of Teratosphaeriaceae I (Fig. 4) revealed nine unidentified RIF strains (CCFEE 5538, 5316, 5505, 5526, 5506, 5537, 5525, 5490, 5543), closely affiliated to Elasticomyces elasticus Selbmann \& Zucconi strain CCFEE 5474. The geographical origin of strains is relatively wide (Table 1) but all habitats are characterized by low average temperatures. Four strains were isolated from antarctic rocks, as among which were CCFEE 5525 and 5526 (04/408 bp ITS sequence difference) isolated from the same rock sample collected in the Antarctic Peninsula. CCFEE 5490 was isolated from Tarn Flat, and CCFEE 5316 from Inexpressible Island, both in Victoria Land area (04/408 ITS sequence difference). CCFEE 5537 and 5538 were isolated from rocks collected in Ladakh and CCFEE 5543 came from Kanij-la Valley; both localities are in the Himalaya's mountain range (11/408 bp ITS sequence difference). The rock specimen from which both CCFEE 5505 and 5506 strains were isolated, was collected in Aconcagua, a mountain located in the Andes. The total ITS difference within the cluster interpreted as $E$. elasticus is $16 / 408$ bp. Within this cluster, all nucLSU sequences are identical to E. elasticus CCFEE 5474 , with the exception of CCFEE 5505, 5525, 5526 and 5538, which have $\sim 0.5 \%$ sequence divergence. Sequence similarity, relative to sequences obtained from $R P B 2$ is up to $96 \%$ for CCFEE 5506, 5525 and 5526, and $95 \%$ for CCFEE 5505, 5537 and 5538. The BT2 sequence of strain CCFEE 5543 shows a similarity of $98 \%$ with Elasticomyces elasticus CCFEE 5474, while others are up to $7 \%$ divergent within the amplified section of BT2. The BT2 sequence from CCFEE 5525 and $R P B 2$ sequences from CCFEE 5316, 5538 and 5543 were not incorporated since sequence data was insufficiently alignable, suggesting that the regions obtained belong to paralogous, pseudo- or precursor genes.

Multilocus analysis endorsed positions of three strains isolated from Alpine rocks, CCFEE 5499, CCFEE 5394 and CCFEE 5492, which cluster in a separate group paraphyletic to Friedmanniomyces endolithicus and Elasticomyces elasticus. The three strains show sequences that resemble each other in all partitions, with a similarity of $96.6 \%, 99.2 \%, 97.5 \%$, and $93 \%$ relative to ITS, nucLSU, BT2 and $R P B 2$ sequences, respectively. Since no generic name has been formerly described yet for these simple, meristematic fungi, a new genus is proposed in the section 'Taxonomy'. Strain CBS 117937, 
isolated from Atazar, a rock formation in the central mountain range of the Iberian Peninsula, was resolved as basal branch to the latter clade.

However the 'Elasticomyces' clade (sensu stricto) was not resolved into a supported bipartition (by posterior probability values [PP]), and thus the given distances to its sister genera rather support the novel genus introduced below.

Four strains isolated from the central mountain range of the Iberian Peninsula (CBS 117941, CBS 117950, CBS 118766, and CBS 119304) and one isolated from limestone formations in Mallorca (CBS 117930) constitute a single monophyletic clade (Clade I-B, Fig. 4) remote from the clade including Friedmanniomyces endolithicus and Elasticomyces elasticus.

Strain CCFEE 5575, isolated from rocks of Foinaven, in the Scottish Highlands, shows $99 \%$ nucLSU sequence similarity and $93 \%$ in RPB2 with Recurvomyces mirabilis Selbmann \& de Hoog (type strain CBS 119434), while BT2 and ITS showed $65 \%$ and $79 \%$ sequence similarity, respectively. The clade is strongly supported by PP but its phylogenetic affiliation remains uncertain because it does not belong to a resolved node. Clade I-C (Fig. 4) comprises three strains of RIF, CBS 136105, CCFEE 5393, which were derived from rocks from two localities on Monte Rosa (Stolenberg and Punta Indren), and CCFEE 5734, which was isolated from Boyl Palace in Cagliari; they possess $98 \%$ ITS sequence similarity with Pseudotaeniolina globosa CBS 109899, a meristematic fungus isolated from monuments in Palermo (Italy). The clade is strongly supported by PP but its phylogenetic affiliation remains uncertain because it belongs to an unresolved node. As Clade I-C, four strains of Clade I-D (Fig. 4) belong to an unresolved node: CBS 136109, CBS 136044, CCFEE 5507, and CCFEE 5457, cluster together at nucLSU and ITS sequence similarities of 98 and 94 \%, respectively. Strains CCFEE 5507 and CBS 136109 have been isolated from a single rock collected in Aconcagua, while CCFEE CBS 136044 and CCFEE 5457 originated from Monte Rosa (Stolenberg locality). Strains CBS 117932 and CCFEE 502 are placed in two different, supported branches in a basal position to the family Mycosphaerellaceae.

An unsupported, but separate cluster comprised CBS 136101 and CBS 136102, two isolates originating from the same rock collected in the Antarctic Peninsula. They cluster as a single species located in a basal position to a highly supported clade of three strains, CCFEE 5569, CBS 118764 and CBS 118355 (Group I-E, Fig. 4). They all originate from the Mediterranean area, i.e. from rocks in Italy and Spain (Oristano, Mallorca and Puebla la Sierra, respectively). NucLSU and ITS sequences of CBS 118764 and CBS 118355 are $99.3 \%$ and $95 \%$ identical, respectively. CCFEE 5569 was resolved distantly remote with $2 \%$ nucLSU sequence divergence while the other gene sections (ITS, BT2 and RPB2) are less than $85 \%$ identical, suggesting the strain may belong to another species. 


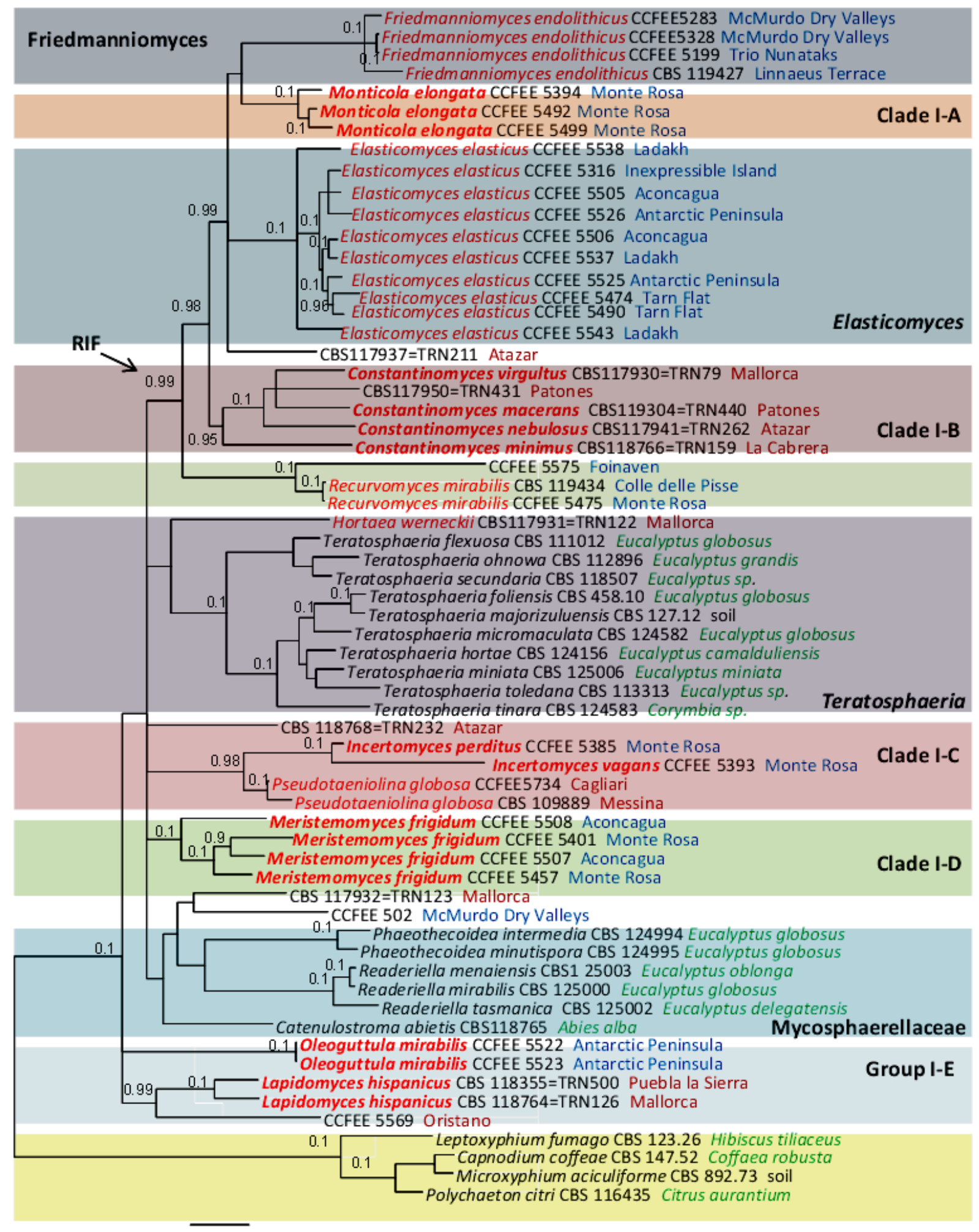

0.09

Fig. 4. Multilocus tree of family Teratosphaeriaceae I, resulting from a Bayesian analysis of nucLSU, RPB2, ITS, BT2 sequence alignment. Bayesian probabilities of $\geq 95 \%$ are provided above corresponding nodes and with thicker lines. Clades and groups are indicated with colored blocks; isolation sources or localities of origin: in blue text are mountain peaks and cold environments, in brown Mediterranean localities and in green plant origins. Strains isolated from rocks are indicated in red text and names of species described here for the first time are in bold. 


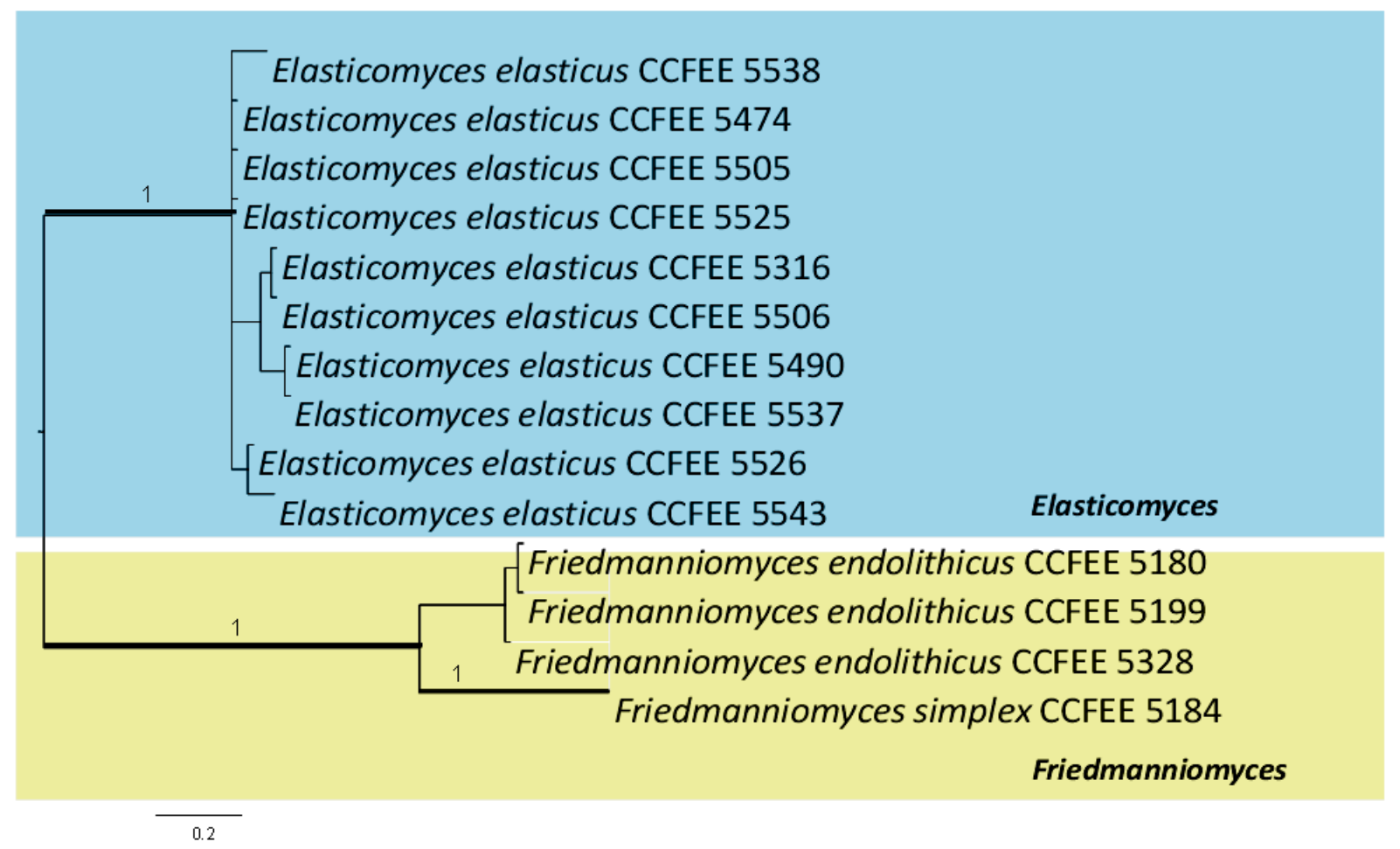

Fig. 5 Species delimitation. Bayesian tree resulting from analysis of ITS sequence alignment of species of Elasticomyces and Friedmanniomyces. Bayesian probabilities of $\geq 95 \%$ are provided above corresponding nodes and in a thicker line. Clades are indicated with colored blocks.

The second largest clade, the Teratosphaeriaceae II (Fig. 6, 7), comprises a large number of rock fungi. In order to delimitate the generic level within and between Teratosphaeriaceae II, Capnodiaceae, Cladosporiaceae, Dissoconiaceae and Mycosphaerellaceae, nucLSU and RPB2 were used, to account for insufficiently alignable ITS and BT2 data over the higher hierarchical ranks (Fig. 6). The manually adjusted alignment contained 66 taxa (including several Capnodiaceae strains used as a outgroup). The resulting phylogeny was very strongly supported by PP, in particular the separation of the ingroup subtrees (families within Capnodiales) and deeper internal branches were almost fully resolved (no polytomies) and resulted in at least four distinguishable clades (Fig. 6). Clade E consists of two strains with uncertain affiliation, while Clade II-F consisted of 7 RIF strains, with Rachicladosporium cboliae (Crous et al. 2009) as the only as yet described species. 


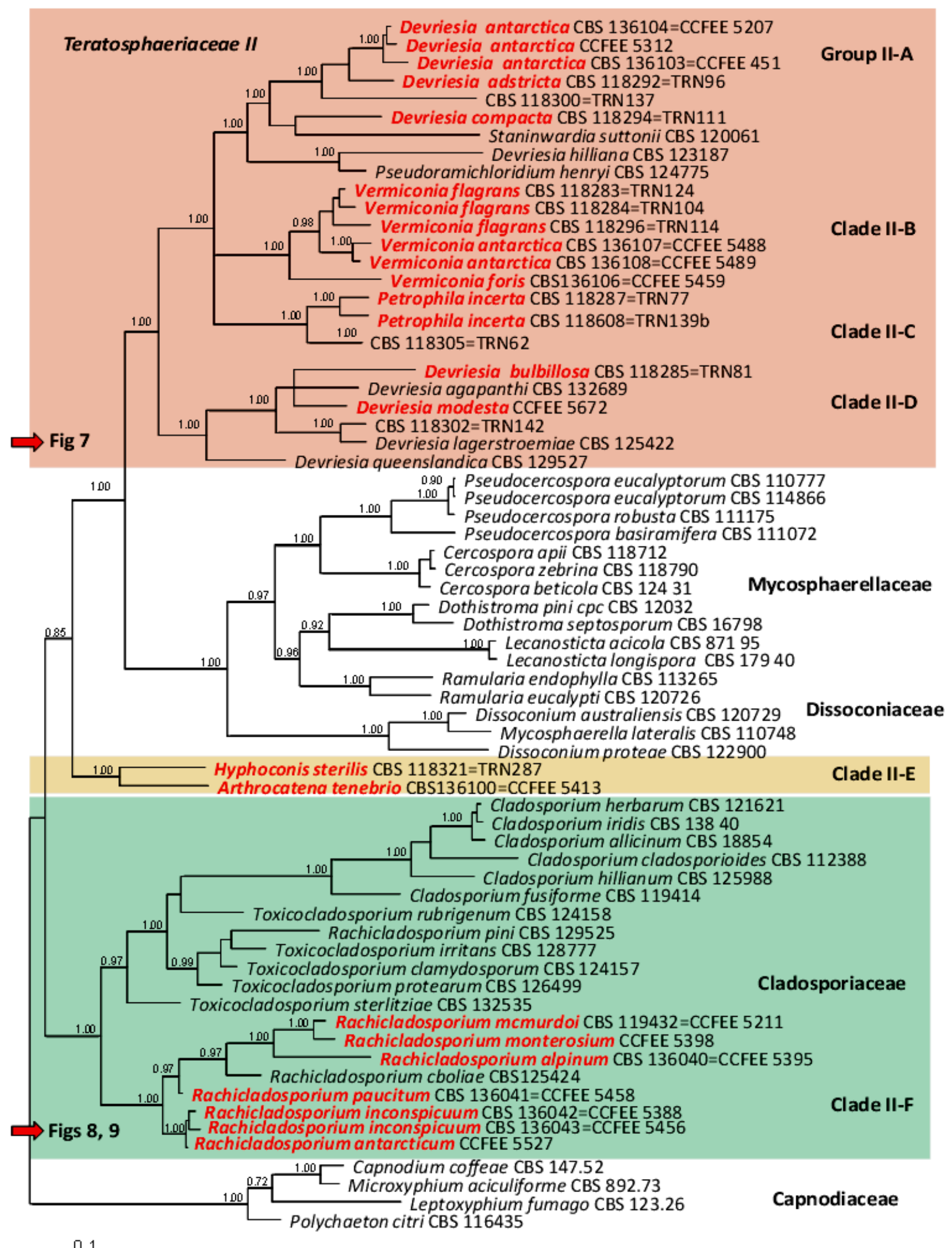

Fig. 6 Two-gene phylogenetic tree of representative families of Capnodiales, resulting from a Bayesian analysis of the nucLSU, RPB2 sequence alignment. Bayesian probabilities of $\geq 95 \%$ are provided above corresponding nodes and in a thicker line. Clades and groups are indicated with colored blocks. Strains isolated from rocks are indicated with red text and species described here for the first time are in bold. Arrows indicate related figures. 

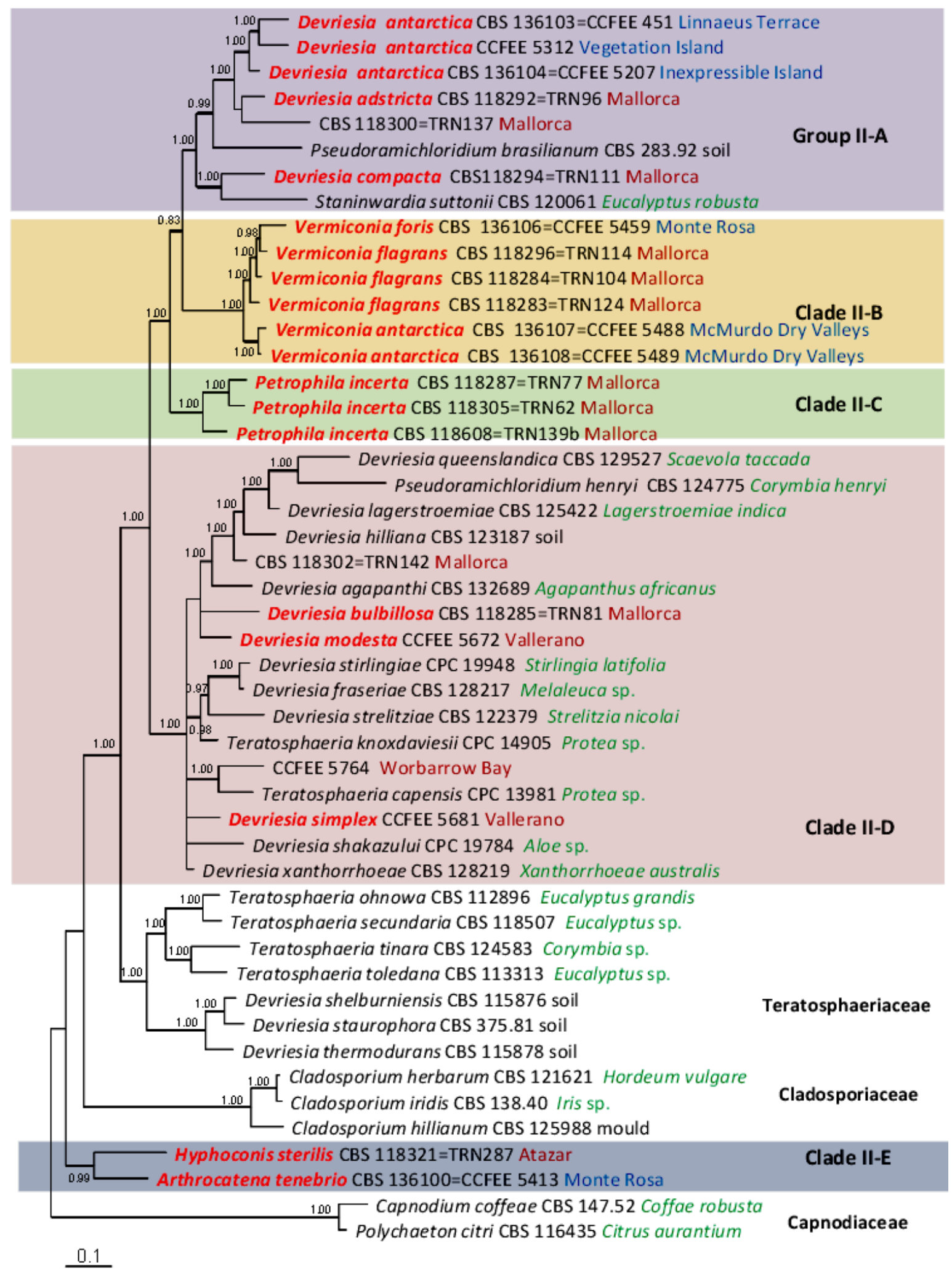

Fig. 7 Four-gene phylogenetic tree of family Teratosphaeriaceae II, resulting from a Bayesian analysis of the nucLSU, RPB2, ITS, BT2 sequence alignment. Bayesian probabilities of $\geq 95 \%$ are provided above corresponding nodes and in a thicker line. Clades and groups are indicated with colored blockswith the isolation source or locality of origin: in blue text are mountain peaks and cold environments, in brown are Mediterranean localities and in green are plant origins. Strains isolated from rocks are in red text and species described here for the first time in bold. 
Affiliations within the Cladosporiaceae are further elaborated in Fig. 8 and 7. RIF nested within the family and phylogenetically associated to Cladosporium (sensu lato), a large genus of ubiquitous saprobes mostly without clear ecological preferences (Bensch et al. 2012) are shown in Fig. 8. In Cladosporium ITS sequences are identical over a large series of species (Fig. 8). 


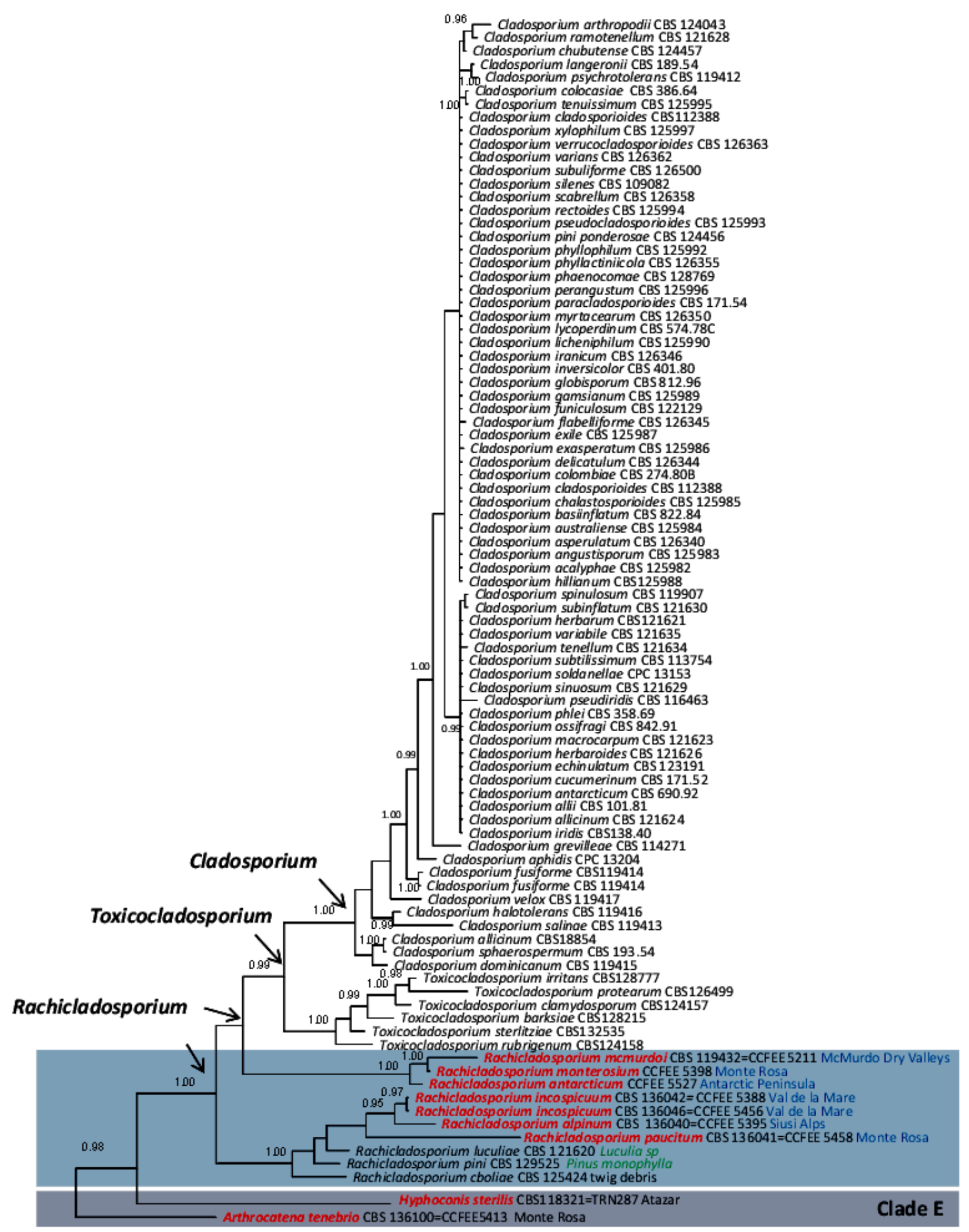

$\underline{0.1}$

Fig. 8 ITS tree of family Cladosporiaceae resulting from Bayesian analysis. Bayesian probabilities of $\geq 95 \%$ are provided above corresponding nodes and in a thicker line. Clades are indicated with colored blocks. Strains isolated from rocks and described here for the first time are indicated in bold followed by locality of origin (blue). In green text are plant species from which Rachicladosporiumspecies have been isolated 
The distantly related novel taxa, distributed over several clades, coincide with the diversity of sampling locations, ranging from the Antarctic Dry Valleys to the Italian Alps. All taxa share a natural habitat influenced by cold climatic conditions. The multilocus tree (Fig. 9) suggests that all sequences in this clade represent individual species of Rachicladosporium, except for CCFEE 5388 and 5456, which show $100 \%$ sequence similarity among their ITS sequences.

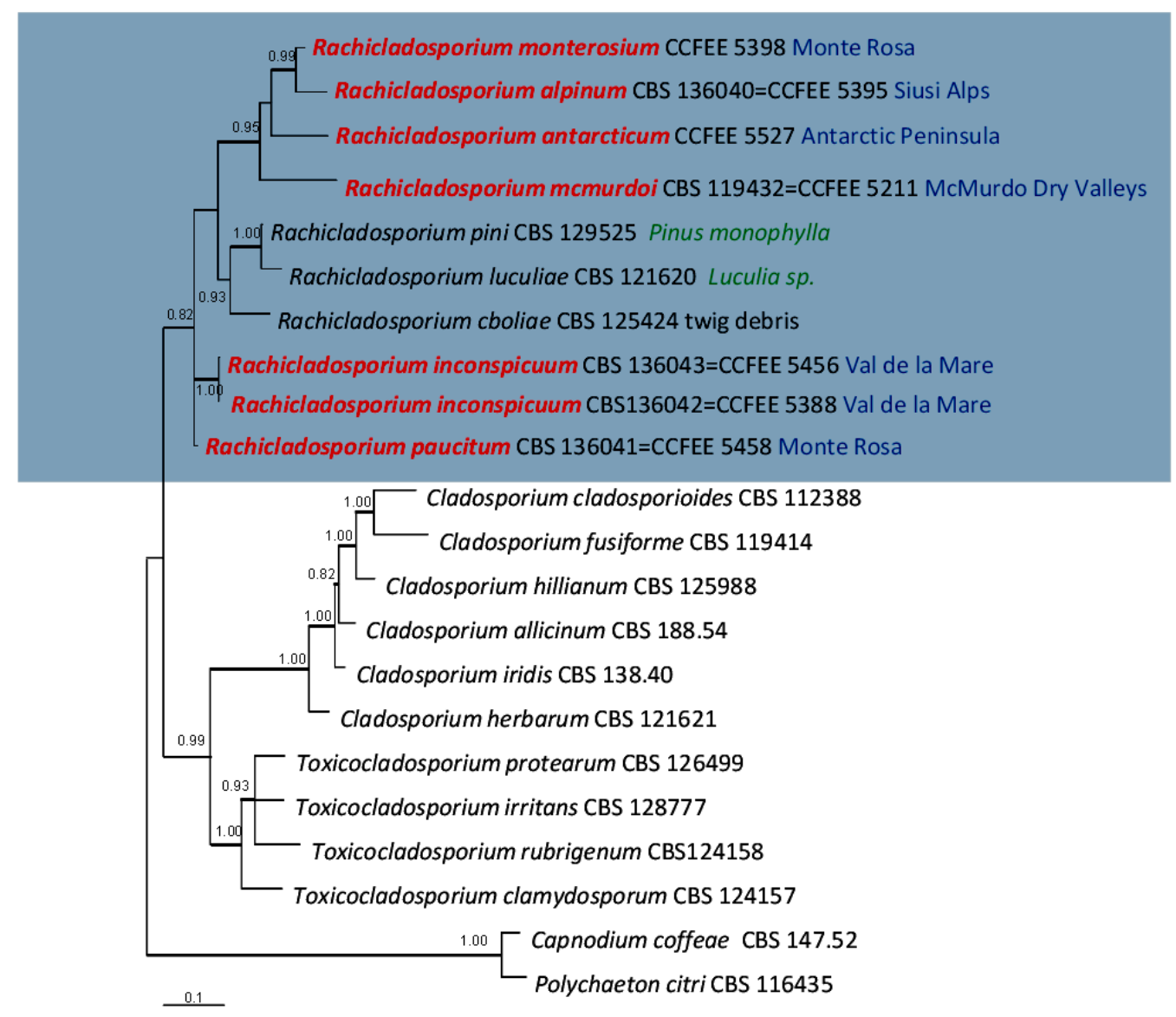

Fig. 9 Four-gene phylogenetic tree of family Cladosporiaceae, resulting from a Bayesian analysis of the nucLSU, RPB2, ITS, BT2 sequence alignment. Bayesian probabilities of $\geq 95 \%$ are provided above corresponding nodes and in a thicker line. Clades and groups are indicated with colored blockswith the isolation source or locality of origin: in blue text are mountain peaks and cold environments, in brown are Mediterranean localities and in green are plant origins. Strains isolated from rocks are in red text and species described here for the first time in bold

More detailed affiliations within Teratosphaeriaceae II are shown in Fig. 7. Sequencing of several Devriesia strains remained unsuccessful even after numerous attempts, and for some of them we 
were unable to produce all necessary sequences. The Devriesia clade was not fully resolved (presence of multiple polytomies), but several branches received high support by PP.

The clade comprises two Teratosphaeria species, the type strain of Pseudoramichloridium, P. henryi CBS 124527, and five RIF unordered among the described species. Species affiliated with Devriesia, a genus accommodating a group of heat-resistant, cladosporium-like (Seifert et al. 2004) and plantassociated fungi (Crous et al. 2009), are included in this group. Group A includes a plant-associated species, Staninwardia suttonii CBS 120061, a fungus isolated from soil, Pseudoramichloridium brasilianum CBS 283.92, and six RIF isolated from Antarctic and Mediterranean rocks. Clade II-B and Clade II-C are two distinct, fully resolved clades with intraspecific ITS divergences between $1 \%$ and $4 \%$, respectively; both clades are exclusively composed of rock-inhabiting fungi, sampled in arid Mediterranean, alpine and antarctic habitats.

Clade $\mathrm{C}$ in particular is composed of strains isolated from rocks sampled in Mallorca only. Hyphoconis sterilis CBS 118321 and Arthrocatena tenebrio CCFEE 5413, introduced below, form a separate highly-supported basal clade (Clade E) with respect to the family Teratosphaeriaceae II.

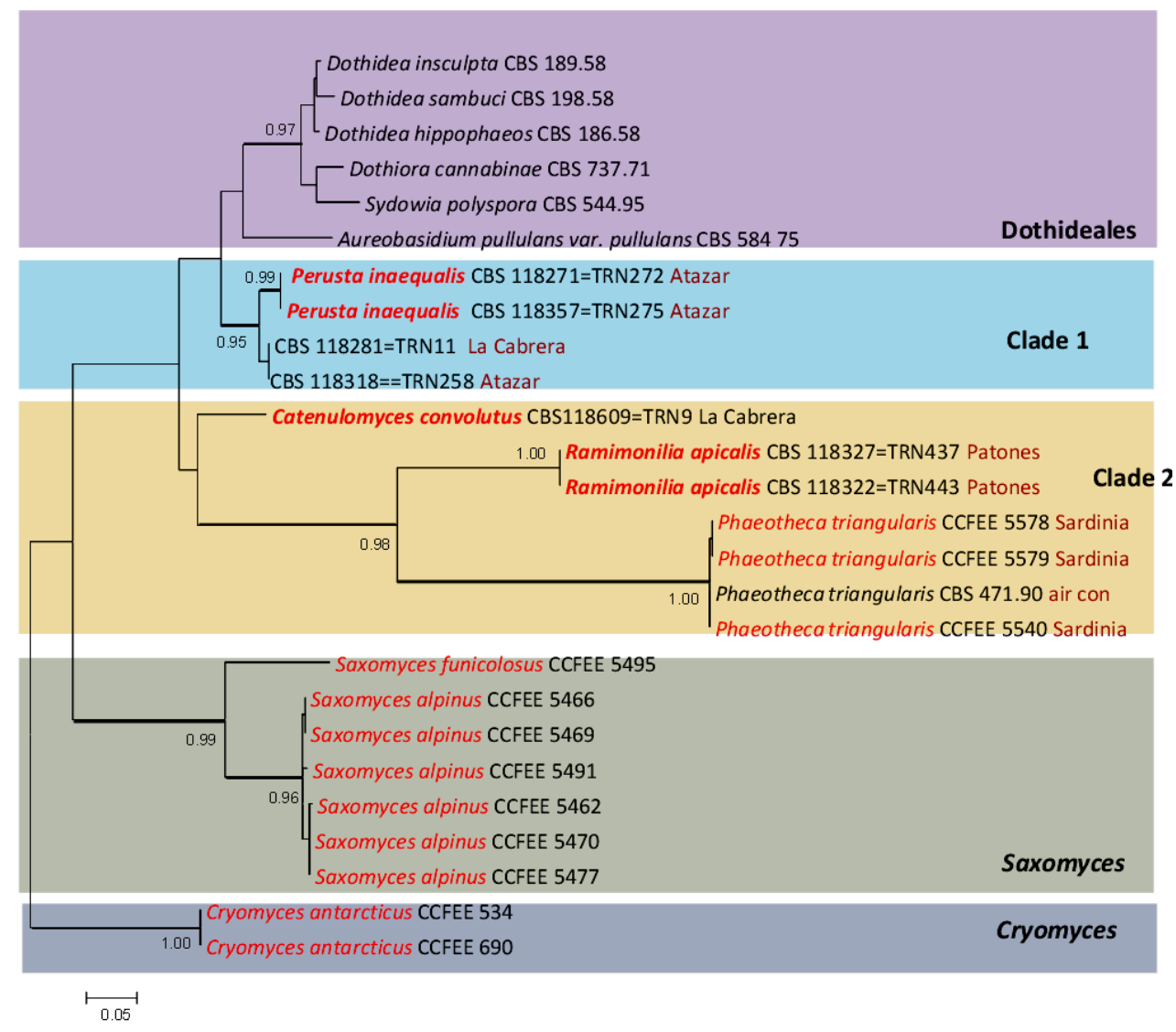

Fig. 10 ITS tree of species of Dothideales: Phaeotheca triangularis, Saxomyces and Cryomyces genera resulting from Bayesian analysis. Bayesian probabilities of $\geq 95 \%$ are provided above corresponding nodes and in a thicker line. Clades are indicated with colored blocks. In brown text Mediterranean localities are indicated. Strains isolated from rocks are indicated in red text and species described here for the first time in names in red bold text. 


\section{TAXONOMY}

Phylogenetic analyses conducted in this study showed that the majority of strains isolated from rock aggregate in undescribed clades which represent taxonomic novelties within the class Dothideomycetes, mainly in the order Capnodiales. The genera and species concerned are described below. In many cases considered strains showed to be scarcely differentiated from a morphological point of view, and therefore taxonomic decisions were based primarily on partial sequences of nucLSU, RPB2, and BT2, and on the complete ITS regions of rDNA.

Descriptions based on 12-wks-old cultures grown on PDA at $15{ }^{\circ} \mathrm{C}$.

Devriesia simplex Selbmann \& Zucconi, sp. nov. MycoBank MB 805278, Fig. 11 A-C.

Holotypus: CBS 137183 = CCFEE 5681, Grotta del Salvatore, Vallerano (VT), Italy. Culture preserved in liquid nitrogen and in dried condition.

Etymology: Named after simple unbranched chains of aseptate conidia.

Diagnosis: colonies growing slowly, up to $10 \mathrm{~mm}$ diam, pale greyish olivaceous, finely velvety, with sharp margin; reverse olivaceous. Conidia arranged in unbranched or poorly branched chains, olivaceous brown, darker than the mycelium, fusiform, guttulate, one-celled (rarely two-celled), liberating easily. Teleomorph unknown.

Notes: As most RIF, CCFEE 5681 showed slow growth and had limited expansion leading to compact mycelium that eventually cracked the agar medium. After prolonged incubation CCFEE 5681 showed conidiation with unbranched acropetal conidia morphologically matching with the genus Devriesia.

Devriesia modesta Isola \& Zucconi, sp. nov. MycoBank MB 805279, Fig. 11 D-F.

Holotypus: CBS 137182 = CCFEE 5672, Grotta del Salvatore, Vallerano (VT), Italy. Culture preserved in liquid nitrogen and in dried condition.

Etymology: named after its scarce exhibition of Devriesia morphology.

Diagnosis: colonies growing slowly, up to $23 \mathrm{~mm}$ diam, pale greyish olivaceous, finely velvety, with sharp margin; reverse olivaceous, with blackish pigment exuded into the agar. Conidia arranged in 
poorly branched chains, pale olivaceous, fusiform with a thin median septum, liberated relatively easily. Teleomorph unknown.

Notes: Devriesia modesta belongs to Clade II-D of Teratosphaeriaceae II (Fig. 7). Some sporulation with undifferentiated conidiophores bearing chains of acropetal conidia were observed. This is a rather common type of sporulation throughout the Teratosphaeriaceae and is found e.g. in Devriesia and Penidiella. The type species of Penidiella, P. kurandae Crous \& Stone was remote in our trees and could not be meaningfully compared. Similarly, the type of Devriesia is D. staurophorum (Kendrick) Seifert \& Nick., found in the Teratosphaeriaceae II in a clade remote to Clade II-D. Given the poor taxon sampling in Dothideomycetidae and the uncertainty in tree topology we consider it inappropriate to introduce a novel genus for Clade II-D. Devriesia species are thermotolerant (Seifert et al. 2004), remarkably many species of Clade II-D originated from habitats in arid climates. The present species was derived from a moist wall of a cave.

Devriesia bulbillosa Egidi \& Zucconi, sp. nov. MycoBank MB 805282, Fig. 11 G-I.

Holotypus: CBS $118285=$ TRN81, Cala San Vicenç, Mallorca, Spain. Culture preserved in liquid nitrogen and in dried condition.

Etymology: named after large, ellipsoidal multicellular structures present in culture.

Diagnosis: colonies growing slowly, up to $20 \mathrm{~mm}$ diam, olivaceous black, compact, with sharp margin; reverse black. Coiled and guttulate hyphae or toruloid hyphae present. Conidia absent. Darkbrown, spherical to broadly ellipsoidal, multicellular bodies present (Fig. $11 \mathrm{H}$, arrow). Teleomorph unknown.

Notes: The species takes an isolated position in Clade II-D of Teratosphaeriaceae II (Fig. 7). Sporulation remained absent despite prolonged incubation under a variety of growth conditions, but the presence of dark, spherical to ellipsoidal, multicellular bodies was observed. Despite the absence of a devriesia-type of sporulation we decided to classify the fungus in the genus Devriesia since all neighbours in Clade II-D did exhibit this morphology and were classified in this genus.

Devriesia compacta de Hoog \& Quaedvlieg, sp. nov. MycoBank MB 805283, Fig. 11 J-L. 
Holotypus: CBS 118294 = TRN111, from rock, Manut II, Mallorca, Spain. Culture preserved in liquid nitrogen and in dried condition.

Etymology: Named after densely packed, barrel-shaped conidia.

Diagnosis: colonies growing slowly, up to $20 \mathrm{~mm}$ diam, greyish olivaceous, compact, finely velvety, with sharp, regular margin; reverse olivaceous black. Conidia pale olivaceous brown, moderately thick-walled, guttulate, catenate, arranged in short chains arising at right angles from hyphae, barrelshaped, strongly coherent (Fig. $11 \mathrm{~L}$, arrow). Teleomorph unknown.

Notes: Like Devriesia adstricta, this species is a member of the approximate group II-A (Figs 4,5$)$ and named after its devriesia-anamorph.

Devriesia adstricta Egidi \& Onofri, sp. nov. MycoBank MB 805284, Fig. 12 A-D.

Holotypus: CBS 118292 = TRN96, from rock, Mallorca, Spain. Culture preserved in liquid nitrogen and in dried condition.

Etymology: named after conidial chains where conidia are densely packed at septa.

Diagnosis: colonies growing slowly, up to $20 \mathrm{~mm}$ diam, black, compact, with sharp, lobed margin; reverse black. Conidia dark brown, guttulate, thick-walled, catenate, ellipsoidal to broadly ellipsoidal with or without a dark median septum, strongly coherent. Sometimes both longitudinal and transversal septa are formed leading to meristematic development (Fig. 12 D, arrow). Teleomorph unknown.

Notes: The species is located in an approximate group II-A of species that are relatively remote from each other (Figs 4, 5). The members of this cluster also are morphologically very different, containing a species displaying compact microdochia (staninwardia-anamorph), with erect sympodial conidiophores (pseudoramichloridium-anamorph), or with ascending, poorly branched chains of acropetal conidia (devriesia-anamorph). Species are found in soil, on plants, and on rock. Consequently neither phylogeny, nor morphology or ecology provides a rationale for classification of this fungus and our choice of Devriesia is mainly for convenience. Phylogenetically the genus can be defined as Clades II-A-D but then comprises some Teratosphaeria species and anamorph-taxa with entirely deviating morphology (Fig. 7). Group II-A obviously suffers from poor taxon sampling and the topology of its phylogenetic tree is likely to change drastically when more species are added. 
Devriesia antarctica Selbmann \& de Hoog, sp. nov. MycoBank MB 805286, Fig. 12 E-G.

Holotypus: CBS 136103 = CCFEE 451, from rock, Linnaeus Terrace, Antarctica. Culture preserved in liquid nitrogen and in dried condition.

Etymology: named after the geographical origin of the strain.

Diagnosis: colonies slow growing and producing finely velvety mycelium, attaining up to $8 \mathrm{~mm}$ in diam in 12 weeks; black in reverse, greyish in obverse; raised centrally, flat near the periphery. Hyphae septate, pale brown, often aggregating into strands. Conidia and chlamydospores absent; teleomorph unknown.

Notes: The species belongs to the Group II-A of Teratosphaeriaceae II (Fig. 7) and, for the same reasons of Devriesia adstricta, we decided to ascribe the fungus to the genus Devriesia. Strains CCFEE 5312 and 5207 show $100 \%$ ITS identity with CCFEE 451. The type culture produced pale brown hyphae. Conidia or chlamydospore-like cells were not observed even after prolonged incubation at different temperatures $\left(6,15,24^{\circ} \mathrm{C}\right)$.

Vermiconia Egidi \& Onofri gen. nov., MycoBank MB 805288

Etymology: Morphology of conidial chains reminiscent of worms.

Colonies growing slowly, dark in reverse, with regular margin. Conidiophores straight, terminating in conidial chains. Conidia resulting from acropetal, holoblastic conidiogenesis, dark brown, growing meristematically, leading to spherical cells with thick walls, developing multiple longitudinal and oblique septa in a later stage.

Type species: Vermiconia foris Egidi \& Onofri

Vermiconia foris Egidi \& Onofri, sp. nov. MycoBank MB 805290, Fig. 12 H-K

Holotypus: CBS 136106 = CCFEE 5459, from rock, Monte Rosa (Italy). Culture preserved in liquid nitrogen and in dried condition.

Etymology: morphology of propagating cultures reminiscent of extraterrestrial worms. 
Diagnosis: colonies growing up to $35 \mathrm{~mm}$, black in reverse, brown in obverse, becoming glistening black near the periphery, compact, lobed, with regular margin. Conidiophores straight, terminating in conidial chains. Conidia resulting from acropetal, holoblastic conidiogenesis, dark brown, guttulate, developing transverse septa at first and after growing meristematically, consisting of spherical cells with thick walls, developing multiple longitudinal and oblique septa in a later stage. Ramoconidia present. Teleomorph unknown.

Notes: This is a member of Clade B in Teratosphaeriaceae II which is composed of rock-inhabiting fungi. Two strains groups can be distinguished, originating from the cold or warm environments (i.e. CCFEE 5459 from the mountain top of Monte Rosa in the Italian Alps), and strains of the next species, which were isolated from natural rock on the Mediterranean island of Mallorca, where high summer temperatures are prevailing.

Vermiconia flagrans Selbmann \& Isola, sp. nov. MycoBank MB 805289, Fig. 12 L-N.

Holotypus: CBS 118296 = TRN114, from rock, Mallorca, Spain. Culture preserved in liquid nitrogen and in dried condition.

Etymology: named after the survival of high summer temperatures prevailing in its natural habitat.

Diagnosis: colonies slow growing up to $35 \mathrm{~mm}$ after 12 weeks of incubations, dark brown, with regular margin. Hyphae septate, pale to dark brown, branched, consisting of cylindrical to elongate cells with thick walls, guttulate. Conidia absent. Teleomorph unknown.

Notes: The species is a member of Teratosphaeriaceae II Clade B and close to Vermiconia foris but consistently separate in all partitions (Fig. 7). Three strains were available which were all isolated from natural rock on the Mediterranean island of Mallorca, and thus the species is likely to take advantage of high temperature stress and is also ecologically different from its psychrophilic counterpart $V$. foris.

Vermiconia antarctica Egidi \& Selbmann, sp. nov. MycoBank MB 807331, Fig. 12 O-R

Holotypus: CBS 136107 = CCFEE 5488, from rock, Battleship Promontory, McMurdo Dry Valleys, Antarctica. Culture preserved in liquid nitrogen and in dried condition. 
Etymology: named after the cold continent the strain was isolated from.

Diagnosis: colonies slow growing up to $10 \mathrm{~mm}$ after 12 weeks of incubation, black in reverse and obverse, hard, elevated centrally and near the periphery, with regular margin. Conidia septate, hyaline to dark brown, consisting of cylindrical cells with thick walls, developing a median septum in an early stage, and after growing meristematically.

Notes: The species is a member of Teratosphaeriaceae II Clade B and close to Vermiconia foris and Vermiconia flagrans but consistently separate in all partitions (Fig. 7). Two strains were available which were isolated from the same rock collected in Antarctica.

Petrophila de Hoog \& Quaedvlieg gen. nov., MycoBank MB 805291

Etymology: named after the rock substrate it was isolated from.

Colonies slow growing, velvety, black in reverse and obverse. Hyphae septate, subhyaline to pale brown, branched, producing cylindrical cells with thick walls. Conidiophores erect, semimacronematous; intercalary conidiogenous cells producing septate conidia.

Type species: Petrophila incerta de Hoog \& Quaedvlieg

Petrophila incerta de Hoog \& Quaedvlieg, sp. nov. MycoBank MB 805292, Fig. 13 A-C

Holotypus: CBS 118608 = TRN139b, from rock, Mallorca, Spain. Culture preserved in liquid nitrogen and in dried condition.

Etymology: named after the rock substrate it was isolated from.

Diagnosis: colonies slow growing, velvety, attaining up to $10 \mathrm{~mm}$ in diam in 12 weeks; black in reverse and obverse. Hyphae septate, subhyaline to pale brown, branched, producing cylindrical cells with thick walls. Conidiophores erect, semi-macronematous, with intercalary conidiogenous cells producing 0-, 1- or 2-septate conidia, with longitudinal septa (Fig. 13 B, arrow). Teleomorph unknown.

Notes: This is a member of Clade $\mathrm{C}$ in Teratosphaeriaceae II which is composed of rock-inhabiting fungi originating from Mallorca and clearly distinct from other clades (Fig. 7). 
Oleoguttula Selbmann \& de Hoog gen. nov., MycoBank MB 805293

Etymology: named after black conidia looking like oil droplets; it is one of the very few sporulating rock-inhabiting fungi.

Diagnosis: colonies black in obverse and reverse, compact, cerebriform, growing slowly, lobed with irregular margin. Hyphae pale to dark brown, branched, consisting of spherical and cylindrical cells with thick walls. Conidia abundant, produced from unicellular, micronematous, terminal or intercalary phialides with wide openings and very short collarettes; conidia aggregating in dense clusters at phialide tips. Conidia pale to dark brown, pyriform, ovoidal, sometimes with one or two median septa.

Type species: Oleoguttula mirabilis Selbmann \& de Hoog

Oleoguttula mirabilis Selbmann \& de Hoog, sp. nov. MycoBank MB 805294, Fig. 13 D-G.

Holotypus: CBS 136102 = CCFEE 5523, from rock, Lachman Crags, Antarctica. Cultures preserved in liquid nitrogen and in dried condition.

Etymology: named after black conidia looking like oil droplets; as one of the very few sporulating rock-inhabiting fungi, the morphology is impressive.

Diagnosis: colonies black in obverse and reverse, compact, cerebriform, growing slowly, up to $7 \mathrm{~mm}$ in diam after 12 weeks, lobed with irregular margin, glistening, moist, buttery. Hyphae pale dark brown, branched, consisting of spherical and cylindrical cells, sometimes guttulate, with thick walls, cells measuring about 5-13 $\times 6-11 \mu \mathrm{m}$, in a later stage developing 2 or more longitudinal and / or oblique septa. Conidia abundant, produced from unicellular, micronematous, terminal or intercalary phialides, with wide openings and very short collarettes; conidia aggregating in dense clusters at phialide tips. Conidia pale to dark brown, smooth- and thick-walled, pyriform, ovoidal, $6.0-11.5 \times$ 3.0-5.5 $\mu \mathrm{m}$, sometimes with one or two median septa. Teleomorph unknown.

Notes: The species clusters to Group I-E (Fig. 4) in Teratosphaeriaceae I and belongs to a long branch in a separate position with respect to Lapidomyces hispanicus. It is one of the few rockinhabiting fungi for which sporulation was observed. 
Hyphoconis Egidi \& Quaedvlieg, gen. nov., MycoBank MB 805295

Etymology: named after absence of sporulation.

Colonies slow growing, black in reverse, velvety and green in obverse, compact, lobed, cerebriform, with regular margin. Mycelium composed of pigmented hyphae, constricted at the septa. Very poorly morphologically characterized, but well distinguished by molecular taxonomy.

Type species: Hyphoconis sterilis Egidi \& Quaedvlieg, sp. nov.

Hyphoconis sterilis Egidi \& Quaedvlieg, sp. nov. MycoBank MB 805295, Fig. 13 H-J.

Holotypus: CBS 118321 = TRN287, from rock, Atazar, Spain. Cultures preserved in liquid nitrogen and in dried condition.

Etymology: named after poor sporulation by hyphal fragments.

Diagnosis: colonies attaining up to $35 \mathrm{~mm}$ in diam in 12 weeks, black in reverse, velvety and green in obverse, becoming dark green or black near the periphery, compact, lobed, cerebriform, with regular margin. Hyphae septate, subhyaline to dark brown, branched, consisting in cylindroid cells, guttulate with smooth thick wall, constricted at the septa, sometime developing transversal septa in a later stage. Conidia and chlamydospores absent; teleomorph unknown.

Notes: The type culture produced brown hyphae only. Conidia or chlamydospore-like cells were not observed even after prolonged incubation at different temperatures $\left(6,15,24{ }^{\circ} \mathrm{C}\right)$.

Catenulomyces Egidi \& de Hoog, gen. nov., MycoBank MB 805312

Etymology: named after its conidial chains.

Colonies slow growing, black in reverse, white or grey in the obverse, becoming brown or black in the periphery, compact, lobed, cerebriform, with irregular margin. Conidia septate with thick walls, developing 2 or more longitudinal and/or oblique septa; bulbil-like, brown, intercalary bodies present.

Type species: Catenulomyces convolutus Egidi \& de Hoog. 
Catenulomyces convolutus Egidi \& de Hoog, sp. nov. MycoBank 805313, Fig. 14 A-D

Holotypus: CBS 118609 = TRN9, from rock, La Cabrera, Spain. Cultures preserved in liquid nitrogen and in dried condition.

Etymology: named after the conidial chains and curly shape of conidia.

Diagnosis: colonies attaining up to $50 \mathrm{~mm}$ in diam in 12 weeks, black in reverse, white or grey in the obverse, becoming brown or black in the periphery, compact, lobed, cerebriform, with irregular margin. Conidia septate, guttulate, hyaline to dark brown, consisting in cylindrical cells, with thick walls, developing 2 or more longitudinal and/or oblique septa; bulbil-like, brown, intercalary bodies present. Teleomorph unknown.

Notes: The isolate clustered in a separate and external position to Ramimonilia apicalis and Phaeotheca triangularis clades (Fig. 10).

Perusta Egidi \& Stielow, gen. nov., MycoBank MB 805314

Etymology: named after the not uniformly burnt-like colour of the colony.

Colonies black in reverse, greyish in obverse, not uniform in color, compact, hard, with irregular margins. Hyphae dark brown, septate, finely guttulate, with thick walls, consisting of round and chained cells with lateral germination. Conidiophores semi-macronematous, conidiogenous cells intercalary. Conidia catenate.

Type species: Perusta inaequalis Egidi \& Stielow

Perusta inaequalis Egidi \& Stielow, sp. nov. MycoBank 805315, Fig. 14 E-H

Holotypus: CBS 118271= TRN272, from rock, Atazar, Spain. Culture preserved in liquid nitrogen and in dried condition.

Etymology: named after the unlilaterally inflating conidium-like cells. 
Diagnosis: colonies growing up to $80 \mathrm{~mm}$, black in reverse, greyish in obverse, darker near the margin, not uniform in color, compact, hard, with irregular margins. Hyphae dark brown, septate, finely guttulate, with thick walls, consisting of round and chained cells with lateral germination (Fig. $14 \mathrm{G}$, arrow). Conidiophores semi-macronematous; conidiogenous cells intercalary. Conidia globose, catenate, dark brown (Fig. 14 F). Teleomorph unknown.

Notes: Clade 1 (Fig. 10) was composed of four strains; strains CBS 118271 and 118357 were isolated from the same locality in the central mountain range in Spain, showing $100 \%$ ITS similarity; for this reason they are ascribed to the same species.

Ramimonilia Stielow \& Quaedvlieg, gen. nov., MycoBank MB 805316

Etymology: the name of the taxon is related to the typically chained disposition of hyphae.

Colonies black in reverse, brown and hairy in obverse, pale brown in the center, becoming black near the periphery, with irregular margin. Hyphae septate, branched, with thick walls, consisting of round and chained cells with apical germination. Teleomorph unknown.

Type species: Ramimonilia apicalis Stielow\& Quaedvlieg

Ramimonilia apicalis Stielow \& Quaedvlieg, sp. nov. MycoBank 805317, Fig. 14 I-K

Holotypus: CBS 118327 = TRN437, from rock, Patones, Spain. Culture preserved in liquid nitrogen and in dried condition.

Etymology: the name of the taxon is related to the typical branched hyphae with apical germination.

Diagnosis: colonies attaining up to $45 \mathrm{~mm}$ in diam in 12 weeks, black in reverse, brown and hairy in obverse, pale brown in the center, becoming black near the periphery, with irregular margin. Hyphae pale to dark brown, septate, guttulate, branched, with thick walls, consisting of round and chained cells with apical germination. Teleomorph unknown.

Notes: Two isolates with identical ITS sequences were found to cluster in a separate and external position to the Phaeotheca triangularis clade (Fig. 10). This is one of the few species that showed optimal growth rate at $24{ }^{\circ} \mathrm{C}$ (Table 5). 
Montincola Selbmann \& Egidi, gen. nov., MycoBank MB 805318

Etymology: inhabitant of the mountain.

Colonies growing slowly, greyish black, with sharp margin, greyish in obverse, raised centrally, flat near the periphery; reverse black. Hyphae thick-walled, branched, elongate, eventually disarticulating into separate cells. Conidiophores micronematous, conidia thallic-arthric, liberating reluctantly. Teleomorph unknown.

Type species: Montincola elongata Selbmann \& Egidi

Montincola elongata Selbmann \& Egidi, sp. nov. MycoBank 805319, Fig. 15 A-D

Holotypus: CBS 136206 = CCFEE 5394, from rock, Stolenberg, Monte Rosa, Italy. Culture preserved in liquid nitrogen and in dried condition.

Etymology: inhabitant of the mountain with elongate conidium-like structures.

Diagnosis: colonies growing slowly, up to $12 \mathrm{~mm}$ of diameter, greyish black, smooth, with sharp margin, greyish in obverse, raised centrally, flat near the periphery; reverse black. Hyphae thickwalled, evenly brown, branched, guttulate, elongate, eventually disarticulating into separate cells. Conidiophores micronematous, conidia thallic-arthric, liberating reluctantly. Teleomorph unknown.

Notes: Three isolates from Monte Rosa clustered in this group (Clade I-A) in an ancestral position to Elasticomyces and Friedmanniomyces (Fig. 4). The strains showed a maximum of $3.4 \%$ ITS mutual difference, which is well below the intraspecific diversity of Elasticomyces elasticus and thus they can be regarded to belong to a single species. Strain CBS $117937=$ TRN211 clustered outside the Montincola clade I-A and was isolated from arid rock formations in central Spain. Another species is concerned.

Constantinomyces Egidi \& Onofri, gen. nov., MycoBank MB 805320

Etymology: genus named after Constantino Ruibal who was one of the first to uncover the stunning diversity of rock-inhabiting fungi. 
Colonies growing slowly, velvety and greyish in obverse, dark in reverse, with regular margin, elevate centrally and near the periphery. Hyphae septate, thick-walled, elongated or cylindrical in later stages; torulose guttulate hypae sometimes present, brown, thick-walled, smooth. Teleomorph unknown.

Type species: Constantinomyces virgultus Egidi \& Onofri

Constantinomyces virgultus Egidi \& Onofri, sp. nov. MycoBank MB 805321, Fig. 15 E-G

Holotypus: CBS $117930=$ TRN79, from rock, Mallorca, Spain. Culture preserved in liquid nitrogen and in dried condition.

Etymology: The species' microscopic morphology is shrub-like.

Diagnosis: colonies growing, velvety dark in reverse, with regular margin. Hyphae pale to dark brown, septate, thick-walled, with apical germination producing elongated, cylindrical hyphae in later stages; torulose hypae sometimes present composed of swollen cells with or without transverse septa, brown, thick-walled, smooth (Fig. 15 G). Teleomorph unknown.

Notes: Clade I-B contains five strains from different arid habitats of rock formations in Spain, which deviate significantly from each other (Fig. 4). Intra-group diversity (15\%) exceeds that of Elasticomyces elasticus, which has been taken as a standard value for maximum intra-specific heterogeneity in the present publication. For this reason all five are treated individually below. The scarcity of characters other than sequences does not provide any support to species distinction.

Constantinomyces macerans de Hoog \& Onofri, sp. nov. MycoBank MB 805322, Fig. 15 H-K

Holotypus: CBS 119304 = TRN440, from rock, Patones, Spain. Culture preserved in liquid nitrogen and in dried condition.

Etymology: named after the mere thin hyphal morphology of the fungus.

Diagnosis: colonies growing slowly up to $52 \mathrm{~mm}$, dark in reverse, velvety, compact and green in obverse, lighter in the center, with regular margin. Hyphae septate, thick-walled, subhyaline to pale brown, often aggregating into strands and loops. Teleomorph unknown. 
Notes: There was no record of conidia or chlamydospore-like cells even after prolonged incubation at different temperatures $\left(6,15,24^{\circ} \mathrm{C}\right)$.

Constantinomyces nebulosus Isola \& Zucconi, sp. nov. MycoBank MB 805323, Fig. 15 L-N

Holotypus: CBS 117941 = TRN262, from rock, Atazar, Spain. Culture preserved in liquid nitrogen and in dried condition.

Etymology: the species name refers to the dark and poorly shaped morphology of the fungus.

Diagnosis: colonies growing slowly up to $30 \mathrm{~mm}$, dark in reverse, velvety, compact and green in obverse, lobed, with regular margin. Hyphae pale to dark brown, guttulate, septate, branched, cylindrical, with thick walls. Sometimes both longitudinal and transversal septa are formed leading to meristematic development (Fig. $15 \mathrm{M}$ ). Teleomorph unknown.

Notes: The type culture produced brown hyphae only and conidia or chlamydospore-like cells were not recorded even after prolonged incubation at different temperatures $\left(6,15,24{ }^{\circ} \mathrm{C}\right)$.

Constantinomyces minimus de Hoog \& Isola, sp. nov. MycoBank MB 805324, Fig. 16 A-C

Holotypus: CBS 118766 = TRN159, from rock, La Cabrera, Spain. Culture preserved in liquid nitrogen and in dried condition.

Etymology: named refers to the scant appearance of the fungus.

Diagnosis: colonies growing slowly up to $31 \mathrm{~mm}$, dark in reverse, velvety, compact and green in obverse, elevate centrally, with regular margin. Hyphae pale to dark brown, septate, branched, elongated, thick-walled with apical germination; teleomorph unknown. Teleomorph unknown.

Notes: Conidia or chlamydospore-like cells were not observed even after prolonged incubation at different temperatures $\left(6,15,24^{\circ} \mathrm{C}\right)$.

Incertomyces Egidi \& Zucconi, gen. nov. MycoBank MB 805325 
Etymology: named after the poor morphological features of the species.

Colonies growing slowly, hyphae thick-walled, enteroblastically proliferating, torulose, septate and cylindrical in later stages producing pyriform or reniform arthroconidia, mostly with a median septum. Teleomorph unknown.

Type species: Incertomyces perditus Egidi \& Zucconi

Incertomyces perditus Egidi \& Zucconi, sp. nov. MycoBank MB 805326, Fig. 16 D-F.

Holotypus: CBS 136105 = CCFEE 5385, from rock, Monte Rosa, Italy. Culture preserved in liquid nitrogen and in dried condition.

Etymology: named after the poor morphological features of the species.

Diagnosis: colonies growing slowly up to $10 \mathrm{~mm}$, dark in reverse, greyish in obverse, dark near the periphery, lobed, with regular margin. Hyphae hyaline and elongated in early stages to dark brown, toruloid, thick-walled, enteroblastically proliferating (Fig. 16 E), septate and cylindrical in later stages. Teleomorph unknown.

Notes: Two isolates from Monte Rosa clustered in Clade I-C, having Pseudotaeniolina globosa strains in ancestral position (Fig. 4). The strains showed a $7 \%$ ITS difference, and they can be regarded to belong to two different species. Strain CCFEE 5734, isolated from monument marbles in Cagliari area, clustered outside the Incertomyces clade I-C.

Incertomyces vagans Egidi \& Selbmann, sp. nov. MycoBank MB 805327, Fig. 16 G-I.

Holotypus: CCFEE 5393, from rock, Monte Rosa, Italy. Culture preserved in liquid nitrogen and in dried condition.

Etymology: the name refers to the scarce morphological differentiation of the taxon.

Diagnosis: colonies growing slowly up to $11 \mathrm{~mm}$, dark in reverse, greyish in obverse, pale brown near the periphery, lobed, with regular margin. Hyphae pale to dark brown, septate, guttulate and thick-walled; pale to dark brown torulose hyphae producing pyriform or reniform arthroconidia, mostly with a median septum. Sometimes both longitudinal and transversal septa are formed leading 
to meristematic development. Chlamydospores in intercalary or terminal positions. Bulbil-like, brown bodies present. Teleomorph unknown.

Notes: The taxon belongs to Clade II-C (Fig. 4) and is positioned in a long branch with respect to Incetiomyces perditus, suggesting that it should be classified in another species.

Meristemomyces Isola \& Onofri, gen. nov. MycoBank MB 805328

Etimology: named after the typical meristematic growth of the fungus.

Colonies growing slowly. Hyphae torulose, septate and thick-walled, from smooth to rugulose, producing thick-walled, pyriform or reniform arthroconidia by disarticulation. Teleomorph unknown.

Type species: Meristemomyces frigidus Isola \& Onofri

Meristemomyces frigidus Isola \& Onofri, sp. nov. MycoBank MB 805329, Fig. 16 J-M.

Holotypus: CBS 136109 = CCFEE 5508, from rock, Aconcagua, Himalaya. Culture preserved in liquid nitrogen and in dried condition.

Etymology: named after the typical meristematic growth and the cold environment from which the strain was isolated.

Diagnosis: colonies growing slowly up to $40 \mathrm{~mm}$, dark in reverse and obverse, smooth, moist, cerebriform, lobed, with irregular margin. Hyphae pale to dark brown, torulose, septate and thickwalled, from smooth to rugulose; hyphae producing arthroconidia by disarticulation. Conidia thickwalled, pyriform or reniform, mostly with a median septum. Incrustations sometimes present. Teleomorph unknown.

Notes: Four strains from different cold environments were found to cluster in Clade I-D (Fig. 4). Intra-group diversity is up to $6 \%$ in ITS, close to the variability rate of Elasticomyces elasticus. For this reason and because ecological niches share similar fatures, all four strains are treated as a single species.

Lapidomyces de Hoog \& Stielow, gen. nov. MycoBank MB 805330 
Etymology: rock-inhabiting fungus.

Colonies growing slowly, compact, black, hard, lobed with regular margin. Hyphae hyaline to pale brown, thick-walled, branched. Teleomorph unknown.

Type species: Lapidomyces hispanicus de Hoog \& Stielow

Lapidomyces hispanicus de Hoog \& Stielow, sp. nov. MycoBank MB 805331, Fig. 17 A-C

Holotypus: CBS 118355 = TRN500, from rock, Puebla la Sierra, Spain. Culture preserved in liquid nitrogen and in dried condition.

Etymology: rock-inhabiting fungus from Spain.

Diagnosis: colonies growing slowly, up to $41 \mathrm{~mm}$ in diameter, black in reverse, velvety, compact and green in obverse, dark near the periphery, hard, cerebriform, lobed with regular margin. Hyphae hyaline to pale brown, thick-walled, branched. Teleomorph unknown.

Notes: Two strains, from continental Spain and the Island of Mallorca, had closely similar sequence profiles and lacked any morphological characteristics except that their colonies on PDA were slightly more expanding than in most other rock-inhabiting Teratosphaeriaceae.

Arthrocatena Egidi \& Selbmann, gen. nov. MycoBank MB 805332

Etymology: named after dark, arthric conidia in chains.

Colonies growing slowly, dark, velvety, compact; hyphae torulose to dark brown, branched, developing longitudinal and oblique septa producing arthroconidia by disarticulation. Conidia dark brown, thick-walled, sometimes with a median septum. Teleomorph unknown.

Type species: Arthrocatena tenebrio Egidi \& Selbmann

Arthrocatena tenebrio Egidi \& Selbmann, sp. nov. MycoBank MB 805333, Fig. 17 D-G.

Holotypus: CBS 136100 = CCFEE 5413, from rock, Punta Indren, Monte Rosa, Italy. Culture preserved in liquid nitrogen and in dried condition. 
Etymology: named after dark, arthric conidia in chains.

Diagnosis: colonies growing slowly, up to $18 \mathrm{~mm}$ in diameter, black in reverse, velvety, and green in obverse, hard and compact, cerebriform, lobed with regular margin. Torulose hyphae dark brown, branched, consisting of spherical and cylindrical cells with thick walls; cells about 3-5 $\mu \mathrm{m}$ wide and developing 2 or more longitudinal and oblique septa. Conidia resulting from arthric secession, relatively abundant, dark brown, thick-walled, initially barrel-shaped, rounding off to nearly spherical after liberation, $6.0-11.5 \times 3.0-5.5 \mu \mathrm{m}$, sometimes with a median septum. Teleomorph unknown.

Notes: The isolate originated from a cold environment and showed a position in a single, long branch of Clade II-E (Figs 4, 5), suggesting the affiliation to a separate species.

Rachicladosporium mcmurdoi Selbmann \& Onofri, sp. nov. MycoBank MB 805334, Fig. 17 H-K.

Holotypus: CBS 119432 = CCFEE 5211, from rock, Battleship Promontory, McMurdo Dry Valleys, Southern Victoria Land, Antarctica. Culture preserved in liquid nitrogen and in dried condition.

Etymology: named after the valley from which the rock was collected.

Diagnosis: colonies attaining up to $12 \mathrm{~mm}$ in diam in 12 weeks, slow growing, black in reverse, velvety and green in obverse, becoming yellow to dark brown near the periphery, compact, with regular margin. Torulose hyphae, subhyaline to dark brown, branched, consisting of cylindrical cells with thick walls, developing 2 or more longitudinal and oblique septa in a later stage leading to meristematic development; 1-2-celled conidia developing by arthric secession. Teleomorph unknown.

Rachicladosporium alpinum Egidi \& Zucconi, sp. nov. MycoBank MB 805334, Fig. 17 L-O.

Holotypus: CBS 136040 = CCFEE 5395, from rock, Siusi Alps, Italy. Culture preserved in liquid nitrogen and in dried condition.

Etimology: named after the mountain chain from which the rock was collected.

Diagnosis: colonies slow growing and producing thin aerial mycelium, attaining up to $80 \mathrm{~mm}$ in diam in 12 weeks; hairy, black in reverse, mostly brown in obverse, sometimes becoming yellowish 
centrally or near the periphery. Hyphae septate, subhyaline to pale brown, 1.5-2.5 $\mu \mathrm{m}$ wide, often aggregating into strands. Conidia and chlamydospores absent. Teleomorph unknown.

Notes: The type culture produced brown hyphae only. Conidia or chlamydospore-like cells were not observed even after prolonged incubation at different temperatures $\left(6,15,24{ }^{\circ} \mathrm{C}\right)$.

Rachicladosporium monterosium Isola \& Zucconi, sp. nov. MycoBank MB 805335, Fig. 18 A-C.

Holotypus: CBS 137178 = CCFEE 5398, from rock, Stolemberg, Monte Rosa, Italy. Culture preserved in liquid nitrogen and in dried condition.

Etymology: named after the mountain Monte Rosa from which the rock was collected.

Diagnosis: colonies slow growing, velvety, attaining up to $24 \mathrm{~mm}$ in diam in 12 weeks; black in reverse, mostly pale brown in obverse, sometimes becoming yellowish centrally, darker near the periphery; elevate with radial folds centrally, flat near the periphery. Hyphae septate, subhyaline to pale brown, 1.5-2.5 $\mu \mathrm{m}$ wide, often aggregating into strands with anastomosis, occasionally forming loops. Conidia and chlamydospores absent. Teleomorph unknown.

Notes: The type culture produced brown hyphae only. Conidia or chlamydospore-like cells were not observed even after prolonged incubation at different temperatures $\left(6,15,24{ }^{\circ} \mathrm{C}\right)$.

Rachicladosporium paucitum Isola \& Egidi, sp. nov. MycoBank MB 805336, Fig. 18 D-G.

Holotypus: CBS 136041 = CCFEE 5458, from rock, Monte Rosa, Italy. Culture preserved in liquid nitrogen and in dried condition.

Etymology: named after poor sporulation by hyphal fragments.

Diagnosis: colonies slow growing and producing thin aerial mycelium, attaining up to $40 \mathrm{~mm}$ in diam in 12 weeks; hairy, black in reverse, mostly pale brown in obverse, sometimes becoming yellowish centrally, darker near the periphery; elevate centrally, flat near the periphery. Hyphae septate, subhyaline to pale brown, branched, consisting of cylindrical cells. Swollen celled hyphae with thick walls, developing 2 or more longitudinal and oblique septa in a later stage leading to meristematic development. Conidia and chlamydospores absent. Teleomorph unknown. 
Notes: The type culture produced brown hyphae only. Conidia or chlamydospore-like cells were not observed even after prolonged incubation at different temperatures $\left(6,15,24{ }^{\circ} \mathrm{C}\right)$.

Rachicladosporium inconspicuum de Hoog \& Stielow, sp. nov. MycoBank MB 805337, Fig. 18 H$\mathrm{J}$.

Holotypus: CBS 136043 = CCFEE 5456, from rock, Monte Rosa, Italy. Culture preserved in liquid nitrogen and in dried condition.

Etymology: the name of the taxon refers to the scarce morphological differentiation observed in the colony.

Diagnosis: colonies slow growing and producing thin aerial mycelium, attaining up to $40 \mathrm{~mm}$ in diam in 12 weeks; hairy, black in reverse, mostly pale brown in obverse, sometimes becoming yellowish centrally, darker near the periphery; elevate centrally, flat near the periphery. Hyphae septate, composed of cylindroid cells, guttulate with smooth thick walls, constricted at the septa, pale brown, 2.5-3.5 $\mu \mathrm{m}$ wide. Conidia and chlamydospores absent. Teleomorph unknown.

Notes: The type culture produced brown hyphae only. Conidia or chlamydospore-like cells were not observed even after prolonged incubation at different temperatures $\left(6,15,24{ }^{\circ} \mathrm{C}\right)$. The ITS sequence of CBS 136043 = CCFEE 5456 shows $100 \%$ similarity with that of CCFEE 5388.

Rachicladosporium antarcticum Onofri \& Egidi, sp. nov. MycoBank MB 805338, Fig. 18 K-M.

Holotypus: type culture CCFEE 5527, from rock, Antarctic Peninsula, Antarctica. Culture preserved in liquid nitrogen and in dried condition.

Etymology: named after the continent from which it was collected.

Diagnosis: colonies slow growing and producing thin aerial mycelium, attaining up to $40 \mathrm{~mm}$ in diam in 12 weeks; flat, hairy, black in reverse, mostly pale brown in obverse, sometimes becoming yellowish centrally, darker near the periphery. Torulose hypae present, branched, guttulate, subhyaline to pale brown, $1.5-2.5 \mu \mathrm{m}$ wide, enteroblastically proliferating. Cylindrical hyphae produced by apical germination. Conidia and chlamydospores absent. Teleomorph unknown.

Notes: The type culture produced brown hyphae only. Conidia or chlamydospore-like cells were not observed even after prolonged incubation at different temperatures $\left(6,15,24{ }^{\circ} \mathrm{C}\right)$. 

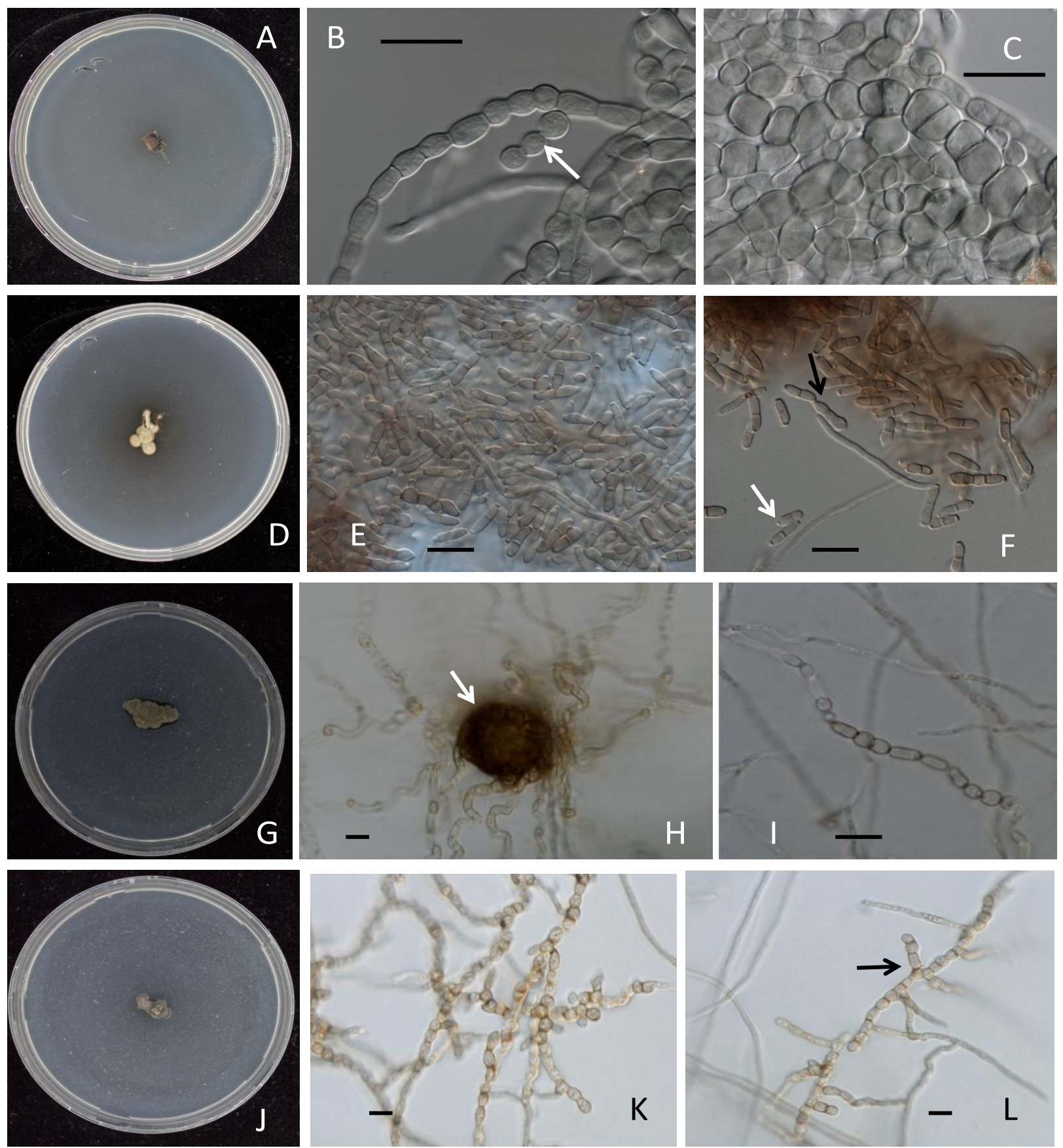

Fig. 11 A-C Devriesia simplex CBS 137183=CCFEE 5681. A: Colony appearance. B: Toruloid pale brown hyphae. C: Septate hyphae. D-F Devriesia modesta CBS 137182=CCFEE 5672. D: Colony appearance. E-F: Pale brown, smoothwalled, fusiform conidia, mostly in unbranched or loosely branched chains. G-I Devriesia bulbillosa CBS $118285=$ TRN81. G: Colony appearance. H: Dark, spherical multicellular body (arrow). I: Branched hyaline hypha. I-J: Devriesia compacta CBS 118294=TRN111. J: Colony appearance. K: Olivaceous brown chaine cells. L: Chains of conidia arising at right angles from hyphae (arrow). Scale bars $10 \mu \mathrm{m}$ 

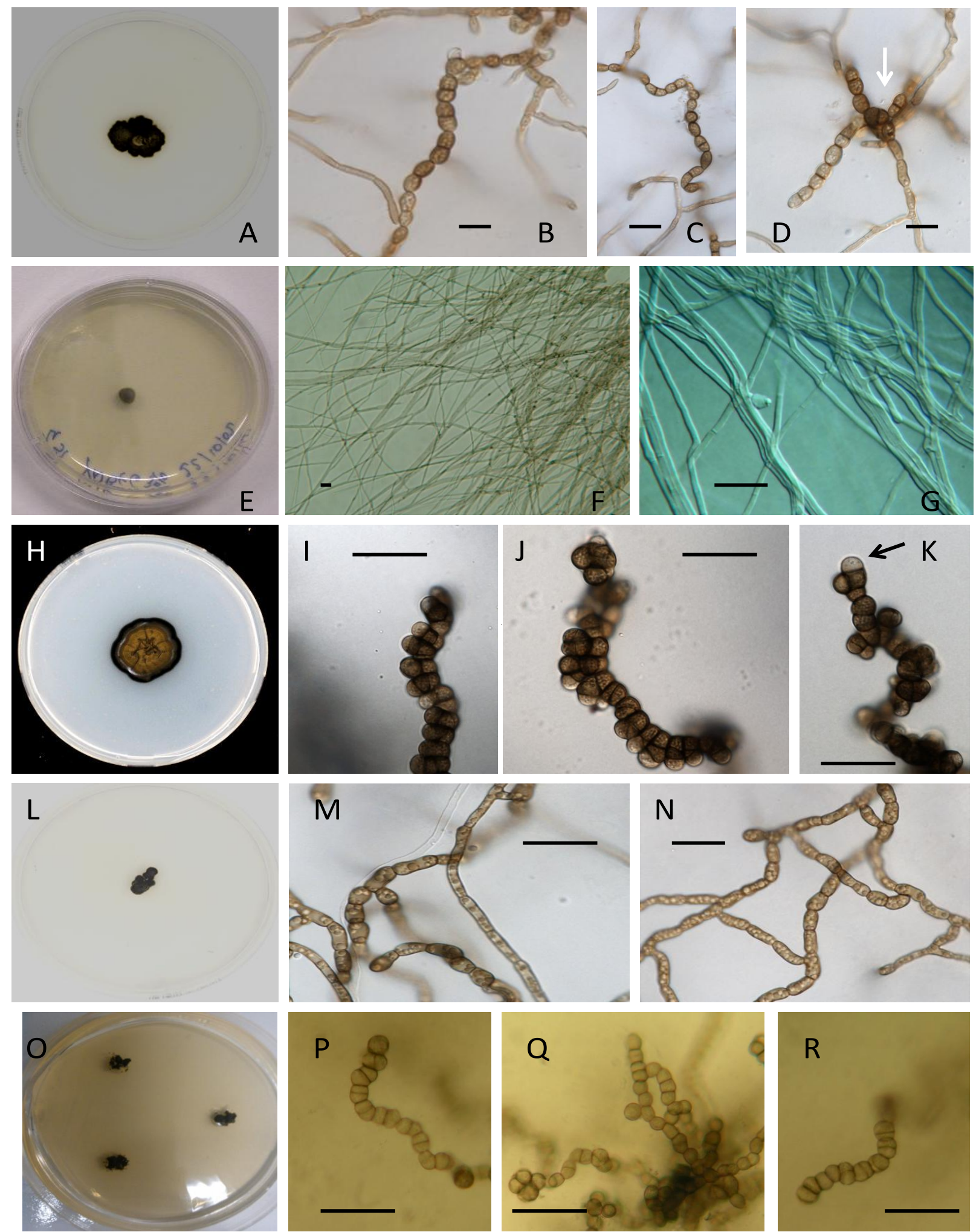

Fig. 12 A-D Devriesia adstricta CBS 118292=TRN96. A: Colony. appearance. B-C: Dark brown, thick-walled, catenate conidia with a dark median septum. D: Meristematic growth. E-G Devriesia antarctica CBS 136103=CCFEE 451. E: Colony appearance. F: Pale brown hyphae organization. G: Hyphae organization in interference contrast microscopy. HK Vermiconia foris CBS 136106=CCFEE 5459. H Colony appearance. I-K: Septate, thick-walled cells showing meristematic growth. L-N Vermiconia flagrans CBS 118296=TRN114. L Strain grown on PDA after 1.5 months of incubation at $15{ }^{\circ} \mathrm{C}$. M-N: Dark brown and thick-walled hyphae. O-R Vermiconia antarctica CBS 136107=CCFEE 5488. O: Colony appearance. P-R: Septate, thick-walled cells showing meristematic growth. Scale bars $10 \mu \mathrm{m}$ 


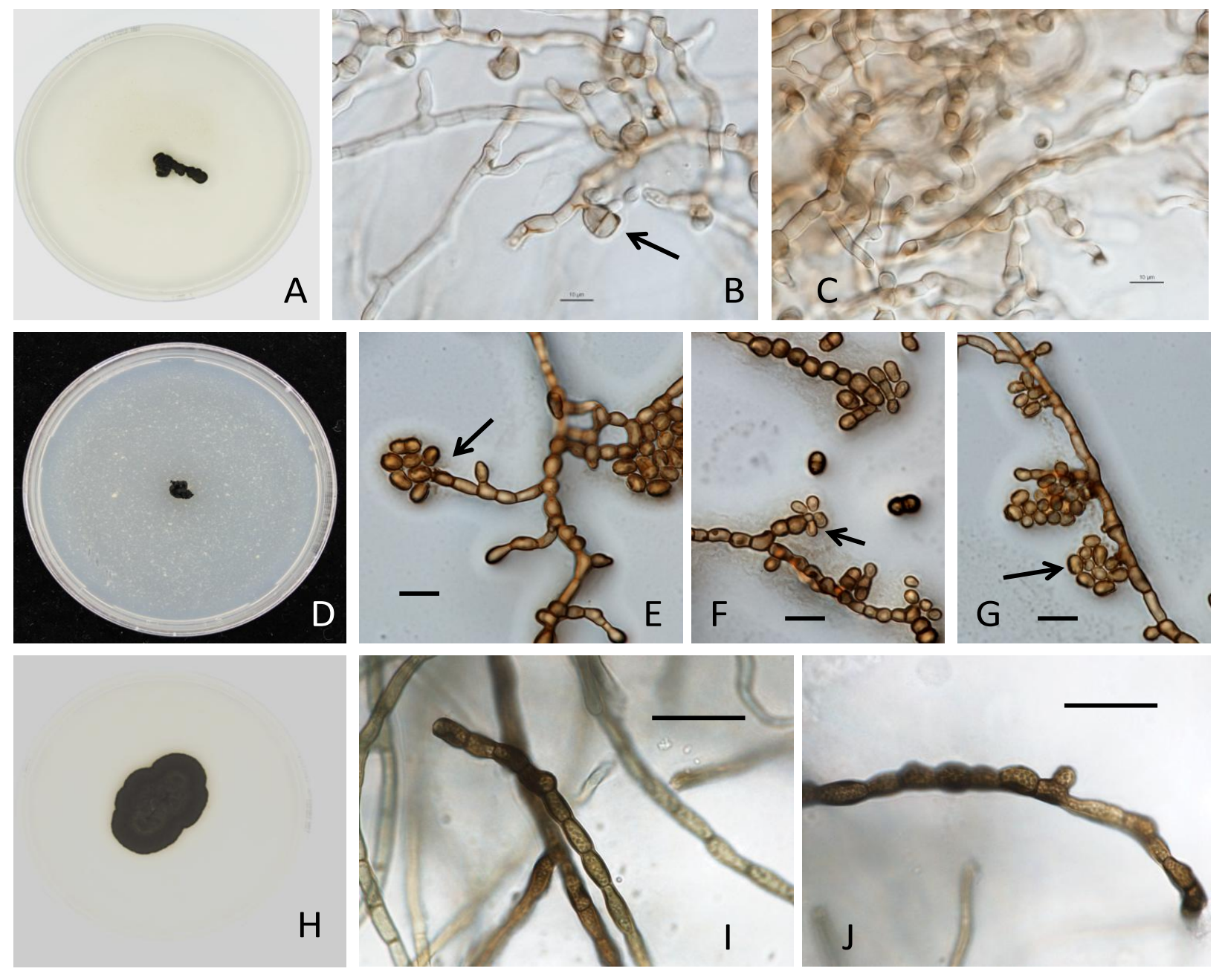

Fig. 13 A-C Petrophila incerta CBS 118608=TRN139b. A: Colony appearance. B: Conidiophores and conidiogenous cells producing 1-septate conidia, with longitudinal septum (arrow). C: Pale brown septate hyphae. D-G: Oleoguttula mirabilis CBS 136102=CCFEE 5523. D Colony appearance. E-G: Phialides with wide openings and very short collarettes and conidia aggregating in dense clusters at phialide tips. H-K Hypoconis sterilis CBS 118321=TRN287. H: Colony appearance. I-J: Septate, dark brown hyphae. Scale bars $10 \mu \mathrm{m}$. 

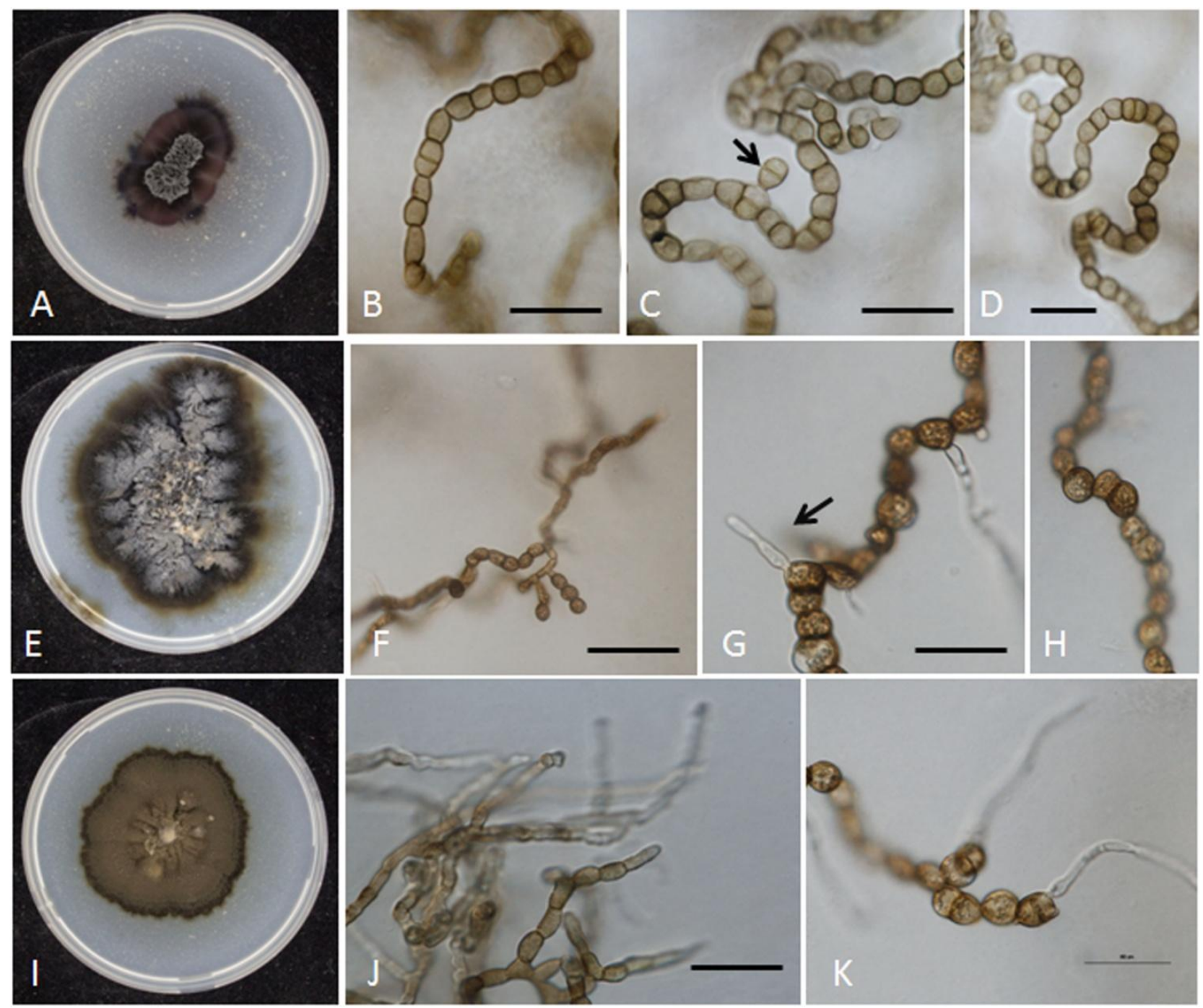

Fig. 14 A-D Catenulomyces convolutus CBS 118609=TRN9. A: Colony appearance. B-D: Mature conidial chains and large conidia with transverse septa (C, arrow). E-H: Perusta inaequalis CBS 118271=TRN272. E: Colony appearance. F: Conidiophores and intercalary conodiogenous with globose, catenate, dark brown conidia. G-H: Guttulate, dark brown hyphae with lateral germination (G, arrow). I-K: Ramimonilia apicalis CBS $118327=$ TRN437. I: Colony appearance. J-K: Hyphae consosting of chained cells, apically germinating. Scale bars $10 \mu \mathrm{m}$ 

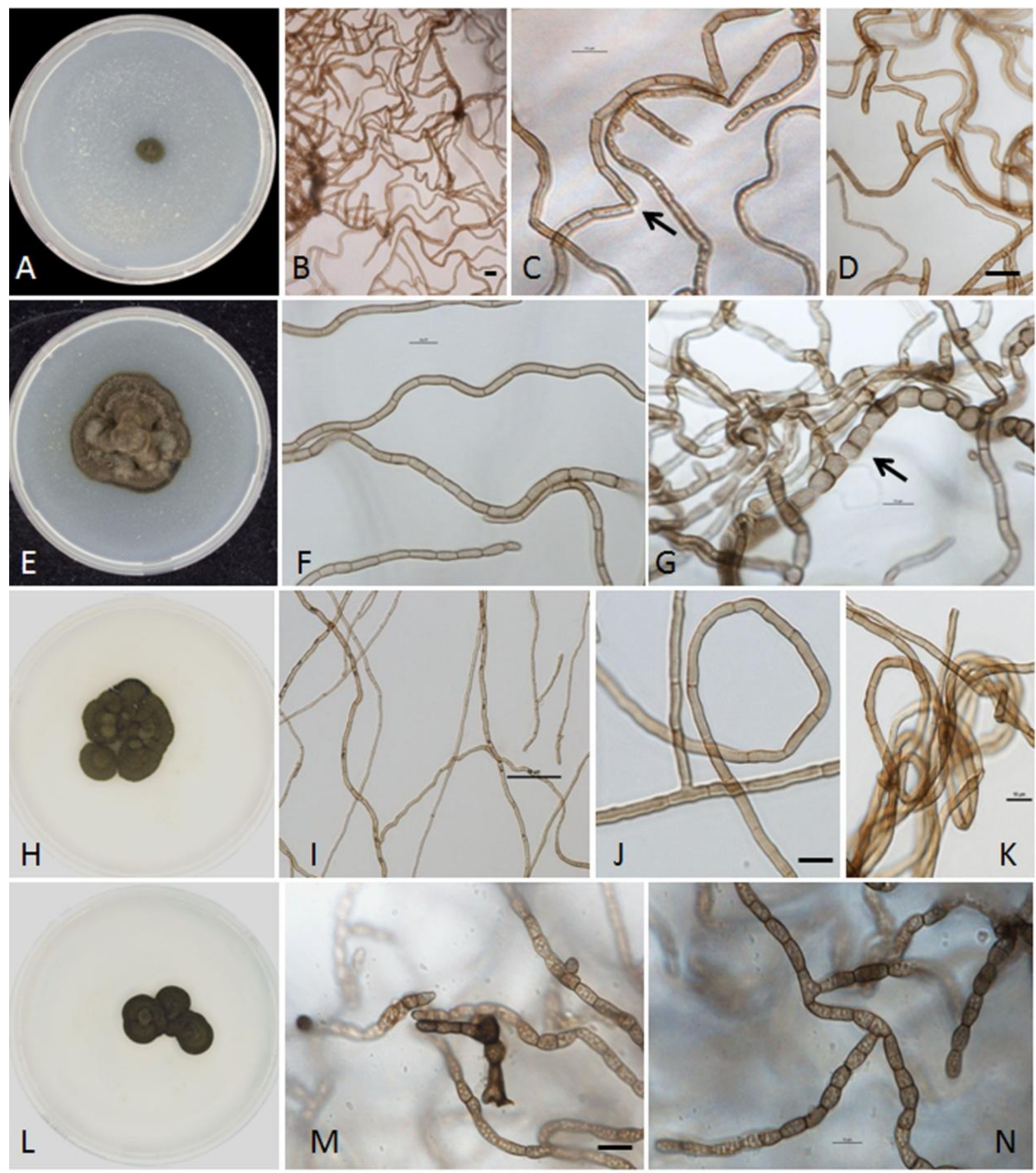

Fig. 15 A-D Monticola elongata CBS 136206=CCFEE 5394. A: Colony appearance. B-D: Micronematous conidiophores and arthroconidia. E-G Constantinomyces virgultus CBS 117930=TRN79. E Colony appearance. $\mathrm{f}$ Septate hyphae. G: Toruloid hyphae. H-K Constantinomyces macerans CBS 119304=TRN440. H: Colony appearance. I-K: Elongated hyphae. L-N Constantinomyces nebulosus CBS 117941=TRN262. L: Colony appearance. M-N: Chained, melanized conidia. Scale bars $10 \mu \mathrm{m}$ 


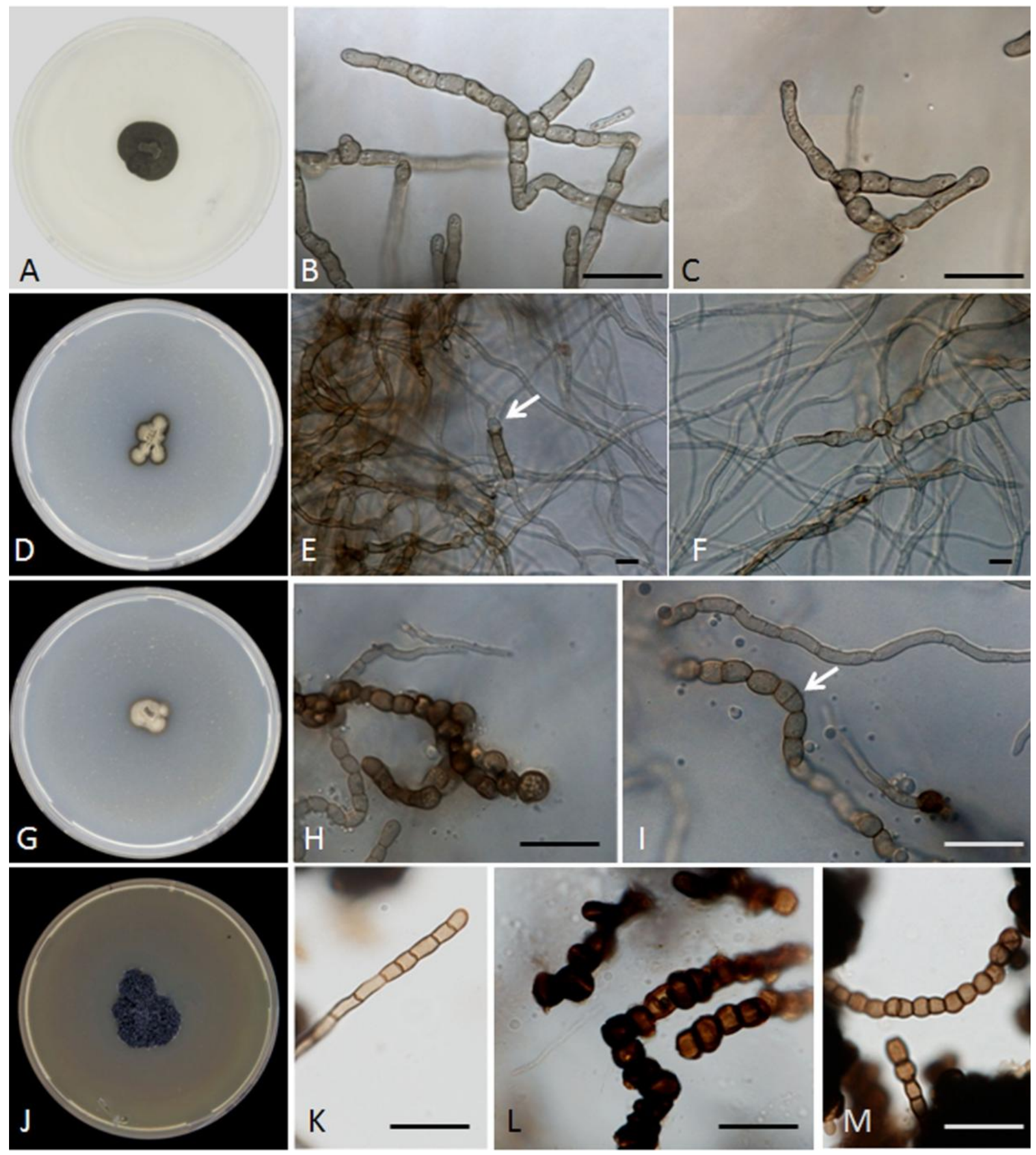

Fig. 16 A-C Constantinomyces minimus CBS 118766=TRN159. A: Colony appearance. B-C: Hyphae with short branches. D-F Incertomyces perditus CBS 136105=CCFEE 5385. D: Colony appearance. E-F: Germination of conidia; hyaline hyphae. G-H Incertomyces vagans CCFEE 5393. G: Colony appearance. H: Dark-brown torulose hyphae. I: Brown, thick walled conidial chains. J-M: Meristemomyces frigidus CBS 136109=CCFEE 5508. J: Colony appearance after 1 month of incubation. K: Brown hypha with septa. 1-m Toruloid and chained hyphal organization. Scale bars $10 \mu \mathrm{m}$ 


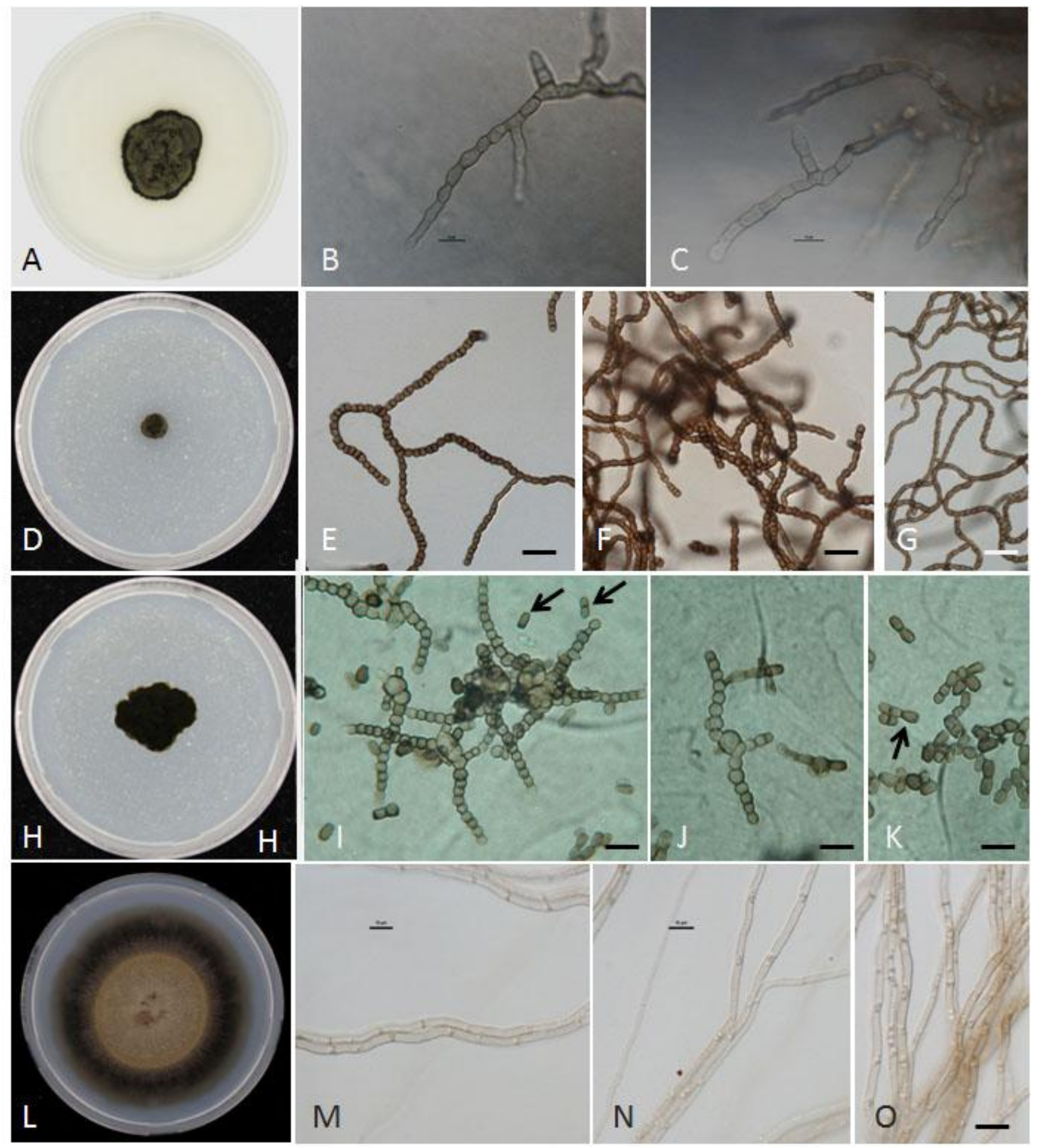

Fig. 17 A-C Lapidomyces hispanicus CBS 118764=TRN126. A: Colony appearance. B-C: Hyphae with short branches. D-G Arthrocatena tenebrio CBS 136100=CCFEE 5413. D: Colony appearance after 3 weeks of incubation. E-G: Thick-walled conidia resulting from arthric conidiogenesis. H-M Rachicladosporium mcmurdoi CBS 119432= CCFEE 5211. H: Strain grown on PDA after 1.5 months of incubation at $15{ }^{\circ} \mathrm{C}$. I-J: Branched and chained conidia. K: Conidia with septae. L-O Rachicladosporium alpinum CBS 136040=CCFEE 5395. L: Colony appearance. M-O: Hyphae septate, subhyaline to pale brown. Scale bars $10 \mu \mathrm{m}$ 

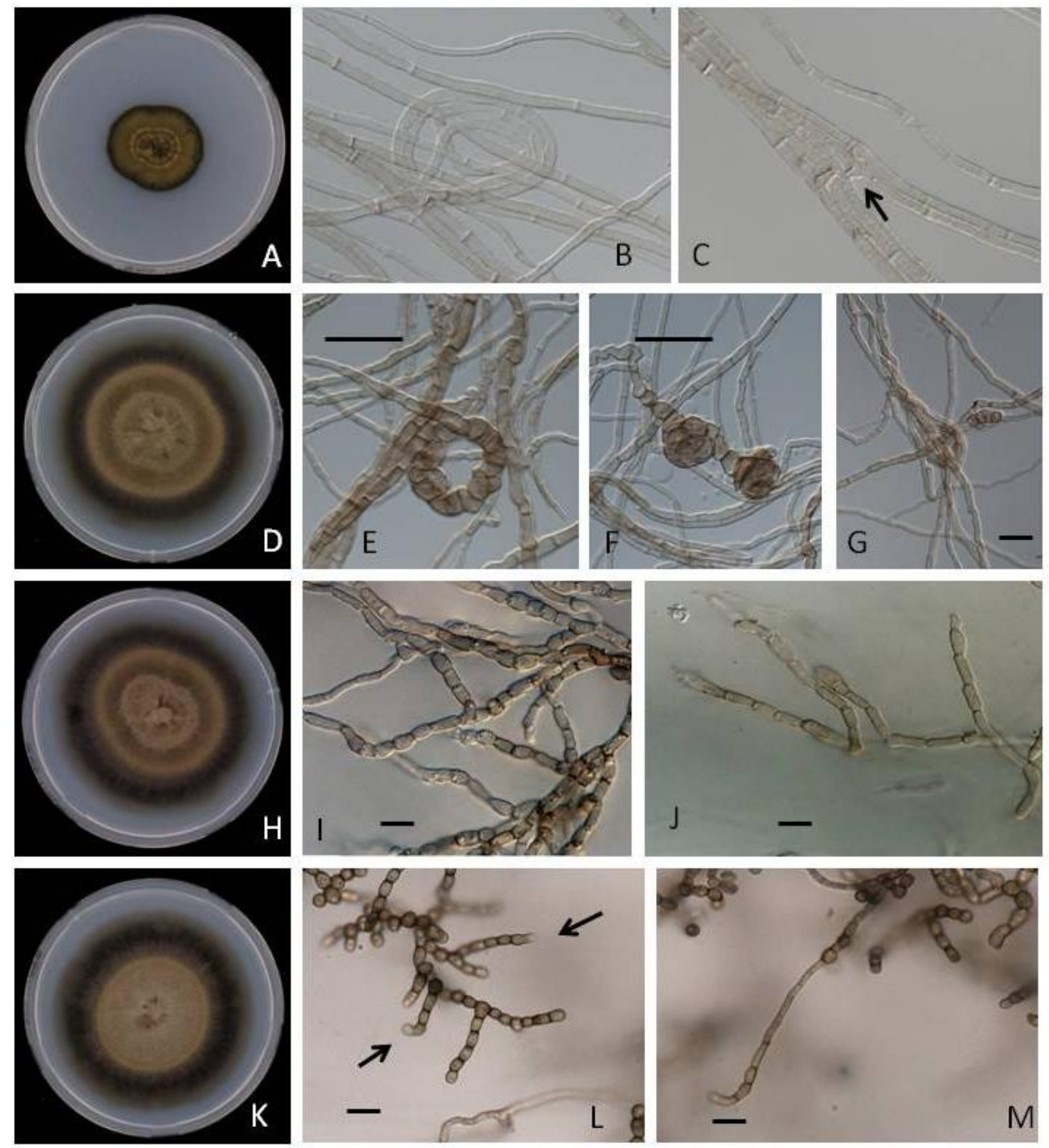

Fig. 18 A-C Rachicladosporium monterosium CBS 137178=CCFEE 5398. A. Colony appearance. B-C: Hyphae septate, subhyaline to pale brown. D-G Rachicladosporium paucitum CBS $136041=$ CCFEE 5458. D: Colony appearance. E-G: Meristematic development of hypae, pale brown. H-J Rachicladosporium inconspicuum CBS 136043=CCFEE 5456. H: Colony appearance. I-J: Chained, elongated, septate hyphae. KM Rachicladosporium antarcticum strain CCFEE 5527. K: Colony appearance. L: Torulose, branched hyphae. M: Germination of hyphae. Scale bars $10 \mu \mathrm{m}$ 


\section{DISCUSSION}

\subsection{MOLECULAR DIVERSITY IN RIF}

A large quantity of strains studied in this paper originated from the classical studies of Ruibal et al. (2004) and Isola (2010), focusing on rock-inhabiting fungi from Mediterranean rocks and from extreme habitats around the globe, respectively. The mountain formations investigated in Spain exhibited semiarid conditions during summers, at high temperatures. Ruibal et al. (2004) sampled pieces of rock with any visible colonization, which were crushed, plated, and RIF were observed to grow directly in culture (isolates with TRN numbers, Table 1). In contrast numerous strains from extremely cold environments included in our study, were collected with macroscopic features, such as cryptendolithic growth in rock crevices (Onofri et al. 1999; Selbmann et al. 2005; 2008; 2013a; b; c). The majority of the isolated strains originated from some of the coldest habitats on the planet, such as Antarctica and to a less extent from mountain formations in the Andes, Alps and Indian Himalayan range. The RIF belonging to the order Chaetothyriales in the Eurotiomycetes (Ruibal et al. 2008) were excluded from the present study. Remarkably, most species causing micropitting on marble and sandstone monuments, reported in numerous studies by e.g. Krumbein et al. (2003) and Sterflinger et al. (1999), are of chaetothyrialean affinity (Onofri et al. 2011).

Taxonomy of RIF is hampered by necessarily inadequate phylogeny due to fragmentary taxon and gene sampling. It is obvious that our understanding of RIF, which thus far have been collected by a limited number of laboratories only, is bound to change enormously in the near future, leading to different taxonomic concepts with every subsequent paper. In view of nomenclatural stability we have tried to be as practical as possible. This has led to different approaches in Teratosphaeriaceae I and Teratosphaeriaceae II. In Teratosphaeriaceae II, RIF are closely akin to described species from habitats other than rock. Over the entire area studied, we witness occurrence of devriesia-types of sporulation, eventually reduced because of poor growth. The group is merged with some occasional taxa with other types of or with no sporulation. The group shares a tendency of extremotolerance by growth on rock or on plants with leathery leaves in arid climates. Within the phylogenetic group Teratosphaeria II we have chosen to classify species according to morphology of prevalent type of sporulation, i.e. mostly in Devriesia. Other generic names where applied where clearly different types of conidiation were prevalent.

In Teratosphaeriaceae I, however, nearly only RIF were found, with only occasional occurrence of species with a plant-inhabiting life style. Fungi were remote from each other, and the backbone of the tree was unresolved. A single cluster of plant-inhabiting Teratosphaeria species figures in the 
tree (Fig. 4), but its position was not supported. Since there was no leading principle to classify the clusters emerging from the unresolved backbone, we describe these clusters as individual genera. Most of them grew very slowly in culture, and lacked characteristic features other than their sequences.

The molecular diversity of RIF in Dothideomycetidae appears quite large. As was already noted by Ruibal (2008), virtually all RIF isolates used in this study showed large quantities of genetic variation between strains, even if these isolates were collected on the same substrate only a few meters away from each other. RIF exhibit very distinct molecular characteristics, even in relatively conserved ribosomal genes. An example of this peculiarity is the clade containing Phaeotheca triangularis, a halophilic fungus originally isolated from a humidifier (de Hoog et al. 1997) and later from saltpans at the Slovenian coast (Zalar et al. 1999) (Fig. 10). Strains CCFEE 5540, 5578 and 5579 (isolated from rock under marine influence in Sardinia) had ITS sequences identical to the type strain. Dothidealean species with an ecology that clearly deviates from that of the majority of known plant-associated fungi often take phylogenetically distant positions. Examples are Ramimonilia apicalis, Catenulomycs convolutus and Perusta inaequalis, which are species described here for the first time and are distant from any sequenced species within the Dothideomycetidae (Fig. 10). This is another indication - along with the high RIF diversity in small sampling areas - that much of the extant fungal diversity is still missing in our trees.

The stunning molecular diversity of RIF strains renders species circumscriptions very problematic. How much should two strains differ in order to be likely representatives of two separate species? To set an approximate standard, we compared the intraspecific heterogeneity of three established species, Friedmanniomyces endolithicus, F. simplex, and Elasticomyces elasticus (Fig. 5). The genus Friedmanniomyces is strictly endemic to one of the coldest regions on Earth, Victoria Land in the Antarctic (Selbmann et al. 2005) (Fig. 5). It forms the fungal component of the cryptendolithic community, having a highly adapted life style in the crevices of rock under extreme environmental conditions, and living in association with very slow-growing lichens algae and cyanobacteria (Onofri et al. 2007; Selbmann et al. 2013b). ITS similarity between type strains of F. simplex (CCFEE 5184) and F. endolithicus (CCFEE 5180) is $93 \%$. About 30 strains of F. endolithicus have now been collected (unpublished data) and the ITS variability within this species is $4.5 \%$.

A similar degree of ITS diversity (4\%) is found in Elasticomyces elasticus. This psychrophilic species has a global distribution on four continents. Separate trees for each single gene of the Elasticomyces cluster have been generated (data not shown) and all showed identical topology; type strain of E. elasticus is CCFEE 5474. Affiliation of strains to Elasticomyces elasticus on the basis of morphology proved to be impossible. The type strain CCFEE 5474 showed a very remarkable, 
auxarthric type of sporulation (Selbmann et al. 2008) which was not observed in any other isolate. CCFEE 5538 showed arthric sporulation with fragmentation of hyphae, but without connectives. Remaining strains were added to the group based on sequence similarity. In part, ITS intraspecific variability varied according to geographic distance: the maximum sequence distance between two strains from Antarctica and Europe is $96 \%$. However, strains CCFEE 5525 and 5526 were isolated from the same rock and even in the same crevice, and still are $1 \%$ different; CCFEE 5474 and 4790 came from a single piece of rock and differ $1.7 \%$. Variability in ITS sequences among all strains from the Antarctic continent is $2 \%$. Andean strains CCFEE 5505 and 5506 were isolated from the same rock and show $99 \%$ similarity, with an overall similarity of $96.5 \%$ for the Antarctic strains. Himalayan strains CCFEE 5537 and 5538 have been isolated from a single rock and show $2 \%$ of distance in ITS sequence, being $3 \%$ distant to CCFEE 5543, a strain originating from a different locality and region of the Himalaya. Globally, internal variability of ITS in this cluster amounts to 4 $\%$.

The above considerations strongly suggest that we should regard Friedmanniomyces endolithicus and Elasticomyces elasticus as two single species, despite their considerable degrees of intraspecific heterogeneity. Particularly the $1 \%$ ITS distance of two strains of E. elasticus from the same crevice in a single piece of rock favours their maintenance in the same species. At this stage we cannot explain the remarkable molecular diversity of RIF. In filamentous fungi this is uncommon and matches, for instance, by Ochroconis, a genus of oligotrophic fungi, frequently found in drinking water and moist domestic environments (Göttlich et al. 2002; Hageskal et al. 2006; Hamada and Abe, 2009; Isola et al. 2013a), where accelerated evolution within the Sympodioventuriaceae has been surmised (Samerpitak et al. 2013). Yet, it was observed that mutational rate may be consistently promoted under stressful conditions and that, irrespective of the causes of their emergence, stressinduced mutations participate in adaptive evolution (Bjedov et al. 2003; Kang et al. 2006). In RIF, where sexual recombination has never been observed and most probably does not take place at all, the stress-increasing mutational rate may represent a genetic strategy for improving survival under pressing conditions. Sexuality is a known driving force in evolution: recombination aids the spread of advantageous traits over populations and purges the genome of deleterious mutations. Clonal organisms gradually but inevitably become extinct because harmful mutations accumulate in the course of time (Muller 1964). Apparently, beyond a particular threshold of stress Muller's ratchet doesn't apply because deleterious mutations are immediately negatively selected without any possibility to accumulate. Clonality, with perpetuation of few super-adapted genotypes, seems to be advantageous for survival. 
The above considerations lead us to the hypothesis that extremophilic RIF are preponderantly clonal and may have accumulated more mutations in non-coding regions than would be expected in species that are purged by sexuality. As a consequence, in the taxonomic species delimitations below we accept larger degrees of sequence heterogeneity than is usual in most other groups of fungi.

\subsection{ECOLOGY OF COLD-LOVING ROCK INHABITING FUNGI}

Five new genera (Arthrocatena, Incertomyces, Meristemomyces, Monticola, Oleoguttula) proposed in this paper have been isolated from rocks from both Antarctic and mountain tops, characterized by extremely cold temperatures for most of the year, adding to the number of previously described coldendemic genera, Cryomyces, Friedmanniomyces, Saxomyces and Elasticomyces. Rock-inhabiting fungi from this particular ecological niche mainly belong to the class Dothideomycetes; from an evolutive perspective, the affinity of these fungi for cold areas could be explained by the evidence that global climate of the period of dothideomycetous RIF rising was characterized by cooler temperatures than today (Gueidan et al. 2011).

From a phyolgenetic point of view, fungi isolated from cold environments exclusively tend to group in separate clusters respect to those with different ecological preferences, suggesting a relationship between adaption to cold environments and phylogenetic isolation of rock inhabiting fungi. In few extreme cases, they can not be addressed to any known order or family. This happens for Saxomyces and Cryomyces, two genera composed by Alpine and Antarctic fungi (Selbmann et al. 2005; 2013), which were found to be remote from known groups and are placed ancestrally within Dothideomycetidae (Fig. 3). Even when addressed to defined families, cold-loving RIF invariably take an isolate position. This character is particularly recurrent in Teratosphaeriaceae I, where most of genera isolated from Antarctica and mountain tops reside: cold-loving fungal species cluster in distinct, separate branches, forming new taxa with no close phylogenetic relationships with plant associated species, which otherwise are very common in this family (Fig. 4). Nevertheless, rockinhabiting fungi isolated from Mediterranean warm areas cluster mainly within Teratosphaeriaceae II family, showing to have a tighter relationship with plant associated fungi (Fig. 7): RIF from Vallerano (Italy) and Mallorca (Spain) belong to new species of Devriesia, a polyphyletic genus comprising soil, plant associated and saprobic species; furthermore, a new genus composed of Mallorcan RIF, Petrophila, is described. Only few strains isolated from cold environments are listed in the family (Devriesia antarctica, Vermiconia foris, Vermiconia antarctica), but still clustering in separate branches respect to plant associated or mediterranean species. Relationship between coldadapted fungi and phylogenetic isolation can be detected in Cladosporiaceae as well: in this family 
RIF have been isolated from Alpine and Antarctic rocks. Six new species belonging to Rachicladoporium, a genus including saprobic and plant pathogenic fungi, are described, but they still form separate clusters composed by fungi isolated from cold environments exclusively (Fig. 8, 9).

The colonisation histories and distributions of cold-loving fungi are likely to be considerably more complex than those of cosmopolitan organisms: some genera of microconial fungi proved to be endemic of one specific region or wider distributed, but still restricted to extremely cold geographical areas. Strains of Friedmanniomyces, Elasticomyces and Recurvomyces have been isolated from Antarctic continent; in particular, Friedmanniomyces is endemic of McMurdo Dry Valleys and Sothern and Northern Victoria Land. The genera Elastycomyces and Recurvomyces show to have a broader distribution: Elastycomyces spp. have been isolated from rocks coming from Andes and Himalaya, the highest mountain tops of the world, while representatives from the genus Recurvomyces have been isolated from Alps. Furthermore, in this paper Oleoguttula is introduced as a new genus from Antarctic continent, while Monticola, Meristemomyces and Incertomyces, form a group of genera originating from Italian Alps. Meristemomyces is a monospecific genus which includes a strain isolated from Himalaya as well. With the exception of Antarctic Elasticomyces strains isolated from lichens, all these fungi act as criptoendolithic organisms, entering the rock porosity to protect themselves to the hostile outside climate and can also be referred to as psychrophilic, as their optimal growth is at $15^{\circ} \mathrm{C}$ and they are not able to grow at temperatures above $25^{\circ} \mathrm{C}$ (Selbmann et al. 2005; 2008). The broad distribution of some genera of cold-loving RIF could be related to ability of RIF to penetrate and consequently actively disrupt rocks, with the production of efficently wind-dispersed propagules, composed by fungi and rock dust clumps.

Dispersal tools, simplified life cycles with no sexual stages, clonal reproduction, slow growth rates and reduced metabolic competences, could be interpreted as winning strategies to thrive in extremely cold areas. On the other side, the absence of phylogenetic affinity with species with a different ecology is a recurrent character in our analyses; moreover, even when worlwide dispersed, coldloving fungi have been isolated from rocks from cold areas exclusively. These features are a precise indication of relatively long-term, not reversible adaptions to survive in chilly, extreme areas: coldloving RIF are scarcely competitive in other environments and are invariably destinated to be restricted to extreme. Future intensified taxon and gene sampling will provide data to confirm or falsify this hypothesis. 


\section{REFERENCES}

Agosta SJ, Klemens JA (2008) Ecological fitting by phenotypically flexible genotypes: implications for species associations, community assembly and evolution. Ecology Letters 11(11):1123-1134.

Altschul SF, Madden TL, Schaffer AA, Zhang J, Zhang Z, Miller W, Lipman DJ (1997) Gapped BLAST and PSI-BLAST: A new generation of protein database search programs. Nucl Acids Res 25: 3389-3402.

Amaral Zettler LA, Gómez F, Zettler E, Keenan BG, Amils R, Sogin ML (2002) Eukaryotic diversity in Spain's River of Fire. Nature 417:137.

Avise JC, Wollenberg K (1997) Phylogenetics and the origin of species. Proc. Natl. Acad. Sci. USA 94:7748-7755.

Berbee ML, Taylor JW (1993) Dating the evolutionary radiations of the true fungi. Canadian Journal of Botany, 71(8):1114-1127.

Bensch K, Braun U, Groenewald JZ, Crous PW (2012) The genus Cladosporium. Stud Mycol 72:1401. doi: 10.3114/sim0003.

Bialek R, Konrad F, Kern J, Aepinus C, Cecenas L, Gonzalez GM, Just-Nübling G, Willinger B, Presterl E, Lass-Flörl C, Rickerts V (2005) PCR based identification and discrimination of agents of mucormycosis and aspergillosis in paraffin wax embedded tissue. J Clin Pathol. 58(11):1180-4.

Bjedov I, Tenaillon O, Gérard B, Souza V, Denamur E, Radman M, Taddei F, Matic I (2003) Stressinduced mutagenesis in bacteria. Science 300:1404-1409. doi: 10.1126/science.1082240

Blackwell M, Hibbett DS, Taylor JW, Spatafora JW (2006) Research Coordination Networks: a phylogeny for kingdom Fungi (Deep Hypha). Mycologia 98(6):829-37

Braams J (1992) Ecological studies on the fungal microflora inhabiting historic sandstone monuments. PhD Thesis. Oldenburg, Germany: Oldenburg University.

Bridge P, Spooner B (2012) Non-lichenized Antarctic fungi: transient visitors or members of a cryptic ecosystem?. Fungal Ecology 5(4):381-394

Burford EP, Kierans M, Gadd GM (2003) Geomycology: fungi in mineral substrata. Mycologist 17(3):98-107.

Butler MJ, Day AW (1998) Fungal melanins: a review. Can .J. Microbiol. 44:1115-1136.

Chertov O, Gorbushina A, Deventer B (2004) A model for microcolonial fungi growth on rock surfaces. Ecol. Model. 177(3): 415-426.

Cooke, RC; Whipps, JM (1993) Ecophysiology of fungi. Blackwell, Oxford. ISBN0-632-02168-3

Costerton, JW, Cheng, KJ, Geesey GG, Ladd TI, Nickel JC, Dasgupta M, and Marrie TJ (1987) Bacterial biofilms in nature and disease. Annu Rev Microbiol 41:435-464 
Crous PW, Braun U, Groenewald JZ (2007a) Mycosphaerella is polyphyletic. Studies in Mycology 58:1-32.

Crous PW, Braun U, Schubert K, Groenewald JZ (2007b) Delimiting Cladosporium from morphologically similar genera. Studies in Mycology 58: 33-56.

Crous PW, Schoch CL, Hyde KD, Wood AR, Gueidan C, de Hoog GS, Groenewald JZ (2009) Phylogenetic lineages in the Capnodiales. Stud Mycol 64:17-47. doi: 10.3114/sim.2009.64.02.

Dadachova E, Casadevall A (2008) Ionizing radiation: how fungi cope, adapt, and exploit with the help of melanin. Curr Opin Microbiol 11:525-531. doi: 10.1016/j.mib/2008.09.013.

De Leo F, Urzì C, Hoog de GS (2003) A new meristematic fungus, Pseudoteniolina globosa. Antonie van Leeuwenhoek 83:351-360.

Del Prado R, Schmitt I, Kautz S, Palice Z, Lücking R, Lumbsch HT (2006) Molecular data place Trypetheliaceae in Dothideomycetes. Mycol. Res. 110:511-520.

de los Ríos A, Wierzchos J, Sancho LG, Grube M, Ascaso C (2002) Microbial endolithic biofilms: a means of surviving the harsh conditions of the Antarctic. Proceedings of the Second European Workshop on Exo/Astrobiology, Graz, Austria, 16-19 September 2002 (ESA SP-518)

Decho, AW (2000) Exopolymer microdomains as a structuring agent for heterogeneity within microbial biofilms. In Microbial Sediments. Riding, R.E., and Awramik, S.M. Eds. Springer Heidelberg, Germany, pp. 9-15.

Dettman JR, Jacobson DJ, Taylor JW (2003) A multilocus genealogical approach to phylogenetic species recognition in the model eukaryote Neurospora. Evolution 57(12):2703-2720.

Diakumaku E, Gorbushina AA, Krumbein WE, Panina L, Soukharjeski S (1995) Black fungi in marble e limestones: an aesthetical, chemical e physical problem for the conservation of monuments. Sci. Tot. Environ. 167: 295-304.

Ehrlich HL (1996) Geomycrobiology. Marcel Dekker Publisher New York. pp.719.

Ehrlich HL (1998) Geomicrobiology: its significance for geology. Earth Sci R 45(1-2): 45-60.

Favero-Longo SE, Gazzano C, Girlanda M, Castelli D, Tretiach M (2011) Physical and chemical deterioration of silicate and carbonate rocks by meristematic microcolonial fungi and endolithic lichens (Chaetothyriomycetidae). Geomicrobiology Journal 28(8): 732-744.

Feibelman TP, Cotty PJ, Doster MA, Michailides TJ (1998) A morphologically distinct strain of Aspergillus nomius. Mycologia 618-623.

Feliner GN Rosselò JA (2007) Better the devil you know? Guidelines for insightful utilization of nrDNA ITS in species-level evolutionary studies in plants. Mol Phylogenet Evol 44(2): 911-919.

Fournier E, Giraud T, Albertini T, Brygoo Y (2005) Partition of the Botrytis cinerea complex in France using multiple gene genealogies. Mycologia 97:1251-1267. 
Frank J, Crous Pw, Groenewald JE, Oertel B, Hyde KD, Phengsintham P, Schroers HJ (2010) Microcyclospora and Microcyclosporella: novel genera accommodating epiphytic fungi causing sooty blotch on apple. Persoonia 24: 93-105. doi:10.3767/003158510X510560.

Friedmann EI (1982) Endolithic microorganisms in the Antarctic cold desert. Science 215:10451053. doi: 10.1126/science.215.4536.1045.

Friedmann EI, Weed R (1987) Microbial trace-fossil formation, biogenous and abiotic weathering in the Antarctic cold desert. Science 236: 703-705.

Gadd GM, Sayer GM (2000) Fungal transformations of metals and metalloids. In Environmental MicrobMetal Interactions, D.R. Lovley ed., American Society for Microbiology, Washington, DC. pp. 237:256.

Gleeson D, Clipson N, Melville K, Gadd G, McDermott F (2005) Characterization of fungal community structure on a weathered pegmatitic granite. Microb. Ecol. 50:360-368.

Golubic S, Friedmann EI, Schneider J (1981) The litobiontic ecological niche, with special reference to microorganisms. J. Sediment. Petrol. 51:475-478.

Gorbushina AA, Krumbein WE (1999) The poikilotrophic micro-organism and its environment Microbial strategies of establishment, growth and survival, In E nigmatic micro-organisms and life in extreme environments. Seckbach, J., Kluwer Academic Press Publishers, Dordrecht Eds, The Netherlands, pp.175-185.

Gorbushina AA, Krumbein W.E. (2000). Subaerial microbila mats and their effects on soil and rock. In Microbial Sediment, R.E. Riding \& S.M. Awramik Eds, Springer-Verlag, Berlin pp 161-170.

Gorbushina AA, Krumbein WE, Volkmann M (2002) Rock surfaces as life indicators: new ways to demonstrate life and traces of former life. Astrobiology 2:203-213. doi:10.1089/15311070260192273

Gorbushina AA (2007). Life on the rocks. Env. Microbiol. 9:1613- 1631.

Gorbushina AA, Kotlova ER, Sherstneva OA (2008) Cellular responses of microcolonial rock fungi to long-term desiccation and subsequent rehydration. Stud Mycol 61:91-97. doi: 10.3114/sim.2008.61.09

Gorbushina AA, Broughton WJ (2009) Microbiology of the atmosphere-rock interface: how biological interactions and physical stresses modulate a sophisticated microbial ecosystem. Annual Rev. Micro. 63:431-450. doi: 10.1146/annurev.micro.091208.073349

Gostinčar C, Muggia L, Grube M (2012) Polyextremotolerant black fungi: oligotrophism, adaptive potential, and a link to lichen symbioses. Front Microbiol 3:390.

Göttlich E, van der Lubbe W, Lange B, Fiedler S, Melchert I, Reifenrath M, Flemming HC, de Hoog GS (2002) Fungal flora in ground-derived public drinking water. Int J Hyg Environ Health 205:269279. 
Gueidan C, Ruibal C, Hoog GS de, Gorbushina A, Untereiner WA, Lutzoni F (2008) A rockinhabiting ancestor for mutualistic and pathogen-rich fungal lineages. Studies in Mycology 61:111119.

Gueidan C, Ruibal C, De Hoog GS, Schneider H (2011) Rock-inhabiting fungi originated during periods of dry climate in the late Devonian and middle Triassic. Fungal biology 115(10):987-996.

Guezennec J, Ortega-Morales BO, Raguenes G, Geesey G (1998) Bacterial colonization of artificial substrate in the vicinity of deep-sea hydrothermal vents. FEMS Microbiol Ecol 26:89-99.

Gunde-Cimerman N, Oren A, Plemenitaš A (2005) Adaptation to life at high salt concentrations in Archaea, Bacteria, and Eukarya. Springer, The Netherlands pp 577.

de Hoog GS (1993) Evolution of black yeasts: possible adaptation to the human host. Antonie van Leeuwenhoek 63:105-109.

Hageskal G, Knutsen AK, Gaustad P, de Hoog GS, Skaar I (2006) Diversity and significance of mold species in Norwegian drinking water. Appl Environ Microbiol 72:7586-7593. doi: 10.1128/AEM.01628-06.

Hamada N, Abe N (2009) Physiological characteristics of 13 common fungal species in bathrooms. Mycoscience 50:421-429. doi: 10.1007/s10267-009-0500-6

Hawksworth D. (1991) The fungal dimension of biodiversity: magnitude, significance, and conservation. Mycological Research 95:641-655.

Hawksworth D (2001) The magnitude of fungal diversity: the 1.5 million species estimate revisited. Mycological research 105(12):1422-1432.

Hibbett D, Binder M, Bischoff JF, Blackwell M, Cannon PF, Eriksson OE, Huhndorf S, James T, Kirk PM, Lücking R, Thorsten Lumbsch H, Lutzoni F, Matheny PB, McLaughlin DJ, Powell MJ, Redhead S, Schoch CL, Spatafora JW, Stalpers JA, Vilgalys R, Aime MC, Aptroot A, Bauer R, Begerow D, Benny GL, Castlebury LA, Crous PW, Dai YC, Gams W, Geiser DM, Griffith GW, Gueidan C, Hawksworth DL, Hestmark G, Hosaka K, Humber RA, Hyde KD, Ironside JE, Kõljalg U, Kurtzman CP, Larsson KH, Lichtwardt R, Longcore J, Miadlikowska J, Miller A, Moncalvo JM, Mozley-Standridge S, Oberwinkler F, Parmasto E, Reeb V, Rogers JD, Roux C, Ryvarden L, Sampaio JP, Schüssler A, Sugiyama J, Thorn RG, Tibell L, Untereiner WA, Walker C, Wang Z, Weir A, Weiss M, White MM, Winka K, Yao YJ, Zhang N (2007) A higher-level phylogenetic classification of the Fungi. Mycological Research 111:509-547.

Hibbett D, Ohmana A, Glotzera D, Nuhna M, Kirkb P, Nilssonc RH (2011) Progress in molecular and morphological taxon discovery in Fungi and options for formal classification of environmental sequences. Fung Biology Rev. 25(1):38-47. doi:10.1016/j.fbr.2011.01.001.

de Hoog GS, Beguin H, Batenburg-van de Vegte WH (1997) Phaeotheca triangularis, a new meristematic black yeast from a humidifier. Antonie van Leeuwenhoek 71:289-295. doi: 10.1023/A:1000156820793.

de Hoog GS, Guarro J, Gené J, Figueras MJ (2000) Atlas of clinical fungi (No. Ed. 2). Centraalbureau voor Schimmelcultures (CBS). 
de Hoog GS (1993) Evolution of black yeasts: possible adaptation to the human host. Antonie van Leeuwenhoek 63:105-109.

Hughes KW, Petersen RH, Lickey EB (2009) Using heterozygosity to estimate a percentage DNA sequence similarity for environmental species' delimitation across basidiomycete fungi. New Phytologist 182(4):795-798.

Huelsenbeck JP, Ronquist F (2001) MRBAYES: Bayesian inference of phylogenetic trees. Bioinformatics 17:754-755. doi: 10.1093/bioinformatics/btg180.

Isola D (2010) Biodiversity, phylogeny and evolution of rock black fungi. Doctoral dissertation, Università degli Studi della Tuscia, Viterbo, Italy. http://dspace.unitus.it/bitstream/2067/1068/1/disola_tesid.pdf

Isola D, Marzban G, Selbmann L, Onofri S, Laimer M, Sterflinger K (2011) Sample preparation and 2-DE procedure for protein expression profiling of black microcolonial fungi. Fungal Biol 115:971977. doi:10.1016/j.funbio.2011.03.001.

Isola D, Selbmann S, de Hoog GS, Fenice M, Onofri S, Prenafeta-Boldú FX, Zucconi L (2013a) Isolation and Screening of Black Fungi as Degraders of Volatile Aromatic Hydrocarbons. Mycopathologia 175(5-6):69-379.

Isola D, Selbmann L, Meloni P, Maracci E, Onofri S, Zucconi L (2013b) Detrimental rock black fungi and biocides: A study on the Monumental Cemetery of Cagliari. In: Rogerio-Candelera MA, Lazzari M, Cano E Science and Technology for the conservation of cultural heritage Eds. CRC Press, London. pp 83-86.

Jacobson ES (2000) Pathogenic roles for fungal melanins. Clin Microbiol Rev. 13(4):708-717.

James TY, Kauff F, Schoch CL, Matheny PB, Hofstetter V, Cox C, Celio G, Gueidan C, Fraker E, Miądlikowska J, Lumbsch HT, Rauhut A, Reeb V, Arnold EA, Amtoft A, Stajich JE, Hosaka K, Sung G-H, Johnson D, O'Rourke B, Crockett M, Binder M, Curtis JM, Slot JC, Wang Z, Wilson AW, Schüßler A, Longcore JE, O'Donnell K, Mozley-Standridge S, Porter D, Letcher PM, Powell MJ, Taylor JW, White MM, Griffith GW, Davies DR, Humber RA, Morton J, Sugiyama J, Rossman AY, Rogers JD, Pfister DH, Hewitt D, Hansen K, Hambleton S, Shoemaker RA, Kohlmeyer J, Volkmann-Kohlmeyer B, Spotts RA, Serdani M, Crous PW, Hughes KW, Matsuura K, Langer E, Langer G, Untereiner WA, Lücking R, Büdel B, Geiser DM, Aptroot A, Diederich P, Schmitt I, Schultz M, Yahr R, Hibbett DS, Lutzoni F, McLaughlin D, Spatafora J, Vilgalys R (2006) Reconstructing the early evolution of the fungi using a six gene phylogeny. Nature 443:818-822.

Johnston CG, Vestal JR (1986). Does iron inhibit cryptoendolithic communities?. Antarct. J. 21: 225-226.

Judd, WS, Campbell CS, Kellogg EA, Stevens P., Donoghue MJ (2002) Plant Systematics: A Phylogenetic Approach, Second Edition. Sinauer Associates Eds, Sunderland: 1-39.

Kang JM, Jovine NM, Blaser JM (2006) A paradigm for direct stress-induced mutation in prokaryotes. FASEB J. 20:2476-2485. doi: 10.1096/fj.06-6209com. 
Katoh K, Toh H (2010) Parallelization of the MAFFT multiple sequence alignment program. Bioinformatics 26:1899-1900. doi: 10.1093/bioinformatics/btq224.

Kemmling A, Kämper M, Flies C, Schieweck O, Hoppert M (2004) Biofilms and extracellular matrices on geomaterials. Env. Geol. 46 (3-4):429-435.

Kirk PM, Cannon PF, Minter DW, Stalpers JA (2008) Dictionary of the Fungi, 10th edition. CABI Pubishing, Wallingford.

Kogej T, Lanisnik-Rizner T, Gunde-Cimermann N (2001) Black yeasts from salterns: the effect of salt on melanization. In: International conference on halophilic microorganisms. Ventosa A, Arahal DR Eds, Sevillia, Spain.

Koufopanou V, Burt A, Szaro T, Taylor JW (2001) Gene genealogies, cryptic species, and molecular evolution in the human pathogen Coccidioides immitis and relatives (Ascomycota, Onygenales). Molecular Biology and Evolution 18(7):1246-1258.

Krumbein WE, Jens K (1981) Biogenic rock varnishes of the Negev desert (Israel), an ecological study of iron and manganese transformation by cyanobacteria and fungi. Oecologia 50:25-38. doi: 10.1007/BF00378791.

Krumbein, WE, Lapo, AV (1996) Vernadsky's Biosphere as a Basis of Geophysiology. Gaia in Action, Science of the living earth. pp.115-134.

Krumbein WE, Brehm U, Gerdes G, Gorbushina AA, Levit GS, Palinska KA (2003) Biofilm, biodictyon, biomat, microbialites, oolites, stromatolites, geophysiology, global mechanisms, parahistology. In Fossil and Recent Biofilms. Krumbein, W.E., Paterson, D.M., and Zavarzin, G.A Eds. Kluwer Academic Publishers, Dordrecht, the Netherlands. pp. 1-27.

Kuramae EE, Robert V, Snel B, Weiß M, Boekhout T (2006) Phylogenomics reveal a robust fungal tree of life. FEMS yeast research 6(8):1213-1220.

Langfelder K, Streibl M, Jahn B, Haase G, Brakhage AA (2003) Biosynthesis of fungal melanins and their importance for human pathogenic fungi. Fun. Gen. Biol.. 38:143-158.

Le Gac M, Hood ME, Fournier E, Giraud T (2007) Phylogenetic evidence of host-specific cryptic species in the anther smut fungus. Evolution 61(1):15-26.

Liu YJ, Whelen S, Hall BD (1999) Phylogenetic relationships among ascomycetes: evidence from an RNA polymerase II subunit. Mol Biol Evol 16:1799-1808.

Liu YJ, Hall BD (2004) Body plan evolution of ascomycetes, as inferred from an RNA polymerase II phylogeny. Proceedings of the National Academy of Sciences of the United States of America, 101(13):4507-4512.

Lumbsch HT, Huhndorf SM (2007) Whatever happened to the pyrenomycetes and loculoascomycetes? Mycol Res. 111(9):1064-74. 
Lutzoni F, Kauff F, Cox C, McLaughlin D, Celio G, et al. (2004). Assembling the fungal tree of life: progress, classification, and evolution of subcellular traits. American Journal of Botany 91:14461480.

Maksaev V, Munizaga F, Zentilli M, Charrier R (2009) Fission track thermochronology of Neogene plutons in the Principal Andean Cordillera of central Chile (33-35 $\mathrm{S})$ : Implications for tectonic evolution and porphyry $\mathrm{Cu}-\mathrm{Mo}$ mineralization. Andean Geol 36:153-171. doi: 10.5027/andgeoV36n2-a01.

Margulis L (1970) Origin of eukaryotic cells. Yale University Press New Haven. pp. 349.

Margulis L (1982) Early life. Science Books Int., Jones and Bartlett Eds, Boston. pp.192.

Marvasi M, Donnarumma F, Frandi A, Mastromei G, Sterflinger K, Tiano P, Perito B (2012) Black microcolonial fungi as deteriogens of two famous marble statues in Florence, Italy. Int. Biodet. Biodeg. 68:36-44

May E, Lewis FJ, Pereira S, Tayler S, Seaward MRD, Allsopp D (1993) Microbial deterioration of building stone - a review. Biodeter Abstr 7: 109-123.

McMenamin, MAS., McMenamin, DS (1994) Hypersea. Life on land Columbia University Press, New York pp. 343.

McNeil M, Roberts AM, Cockerell V, Mulholland V (2004) Real-time PCR assay for quantification of Tilletia caries contamination of UK wheat seed. Plant Path. 53(6):741-750.

Moncalvo JM, Lutzoni FM, Rehner SA, Johnson J, Vilgalys R (2000) Phylogenetic relationships of agaric fungi based on nuclear large subunit ribosomal DNA sequences. Systematic biology 49(2):278-305.

Moses V, Holm-Hansen O, Calvin M (1959) Non photosynthetic fixation of carbon dioxide by three microorganisms, J Bacteriol 77:70-78

Muggia L, Hafellner J, Wirtz N, Hawksworth DL, Grube M (2008) The sterile microfilamentous lichenized fungi Cystocoleus ebeneus and Racodium rupestre are relatives of plant pathogens and clinically important dothidealean fungi. Mycological Research 112: 50-56.

Muller HJ (1964). The relation of recombination to mutational advantage. Mutat. Res. 1:2-9.

Nai C,Wong HY, Pannenbecker A, BroughtonWJ, Benoit I, de Vries RP, Gueidan C, Gorbushina AA (2013) Nutritional physiology of a rock-inhabiting, model micro-colonial fungus from an ancestral lineage of the Chaetothyriales (Ascomycetes). Fungal Genet Biol 56:54-66. doi:10.1016/j.fgb.2013.04.001.

Nelsen MP, Lücking R, Grube M, Mbatchou JS, Muggia L, Rivas Plata E, Lumbsch HT (2009). Unravelling the phylogenetic relationships of lichenised fungi in Dothideomyceta. Studies in Mycology 64: 135-144.

Neu TR (1996) Significance of bacterial surface-active compounds in interaction of bacteria with interfaces. Microbiol Rev. 60(1): 151-166. 
Nienow JA, Friedmann EI (1993) Terrestrial litophytic (rock) communities. Antarctic Microbiology Wiley-Liss Eds., New York. pp 343-412.

Nilsson RH, Kristiansson E, Ryberg M, Hallenberg N, Larsson KH (2008) Intraspecific ITS variability in the kingdom Fungi as expressed in the international sequence databases and its implications for molecular species identification. Evolutionary bioinformatics online 4:193.

Nilsson RH, Ryberg M, Abarenkov K, Sjökvist E, Kristiansson E (2009) The ITS region as a target for characterization of fungal communities using emerging sequencing technologies. FEMS Microbiology Letters 296(1):97-101.

Ninari N, Berliner PR (2002) The role of dew in the water and heat balance of bare loess soil in the Negev Desert: quantifying the actual dew deposition of the soil surface. Atmos Res. 64:323-34.

O’Brien HE, Parrent JL, Jackson JA, Moncalvo JM, Vilgalys R (2005) Fungal community analysis by largescale sequencing of environmental samples. Applied and Environmental Microbiology 71(9):5544-5550.

O’Donnell K, Cigelnik E (1997) Two divergent intragenomic rDNA ITS2 types within a monophyletic lineage of the fungus Fusarium are nonorthologous. Mol Phylo Evol 7:103-116. doi: 10.1006/mpev.1996.0376.

Omelon, C, Pollard W, Ferris F (2006) Chemical and ultrastructural characterization of high arctic cryptoendolithic habitats. Geomycrobiol. J. 23(3-4): 189-200.

Onofri S, Pagano S, Zucconi L, Tosi S (1999) Friedmanniomyces endolithicus (Fungi, Hyphomycetes), anam-gen and sp nov, from continental Antarctica. Nova Hedwigia 68:175-182.

Onofri S, Selbmann L, de Hoog GS, Grube M, Barreca D, Ruisi S, Zucconi L (2007) Evolution and adaptation of fungi at the boundaries of life. Adv Space Res 40:1657-1664. doi:

10.1016/j.asr.2007.06.004.

Onofri S, Barreca D, Selbmann L, Isola D, Rabbow E, Horneck G, de Vera JPP, Hatton J, Zucconi L (2008) Resistance of Antarctic black fungi and cryptoendolithic communities to simulated space and Mars conditions. Stud Mycol 61:99-109.

Onofri S, Anastasi A, Del Frate G, Di Piazza S, Garnero N, Guglielminetti M, Isola D, Panno L, Ripa C, Selbmann L, Varese GC, Voyron S, Zotti M, Zucconi L (2011) Biodiversity of rock, beach and water fungi. Plant Biosyst 145:1-10. doi: 10.1080/11263504.2011.633117.

Onofri S, de la Torre R, de Vera JP, Ott S, Zucconi L, Selbmann L, Scalzi G, Venkateswaran KJ, Rabbow E, Sánchez Iñigo FJ, Horneck G (2012) Survival of rock-colonizing organisms after 1.5 years in outer space. Astrobiology 12:508-516.

Onofri S, Selbmann L, Isola D, Zucconi L (2014) Rock-inhabiting fungi and their role in deterioration of stone monuments in the Mediterranean area. Plant Biosyst. doi: 10.1080/11263504.2013.877533. 
Ortega-Morales BO, Lopez-Cortes A, Hernandez-Duque G, Crassous P, Guezennec J (2001) Extracellular polymers of microbial communities colonizing ancient lime- stone monuments.

Microb Growth Biof Part A 336: 331-339

Øvstedal DO, Lewis Smith RI (2001) Lichens of Antarctica and South Georgia. A Guide to their identification and ecology. Studies in Polar Research. Øvstedal DO, Lewis Smith RI, Eds. Cambridge University Press. pp. 1-35.

Page RDM. (1996) Treeview: An application to display phylogenetic trees on personal computers. Comput. Appl. Biosci. 12: 357-358

Palmer RJ, Friedman EI (1988) Incorporation of inorganic carbon by Antarctic cryptoendolithic fungi. Polarforschung 58:189-191

Pinna D, Salvadori O (1999) Biological growth on Italian monuments restored with organic or carbonatic compounds. Atti del Convegno "Of microbes and art", Firenze 16-19 giugno 1999, pp. 149-154.

Posada D, Crandall KA (1998) Modeltest: testing the model of DNA substitution. Bioinformatics 1: 817-818. doi: 10.1093/bioinformatics/14.9.817.

Potts M (1999) Mechanisms of desiccation tolerance in cyanobacteria. Eur. J. Phycol. 34: 319-328.

Plummer C, McGeary D (1996) Physical geology: With interactive plate tectonics. Seventh Edition ISBN 13: 9780697266767.

Pringle A, Baker DM, Platt JL, Ware JP, Latge JP, Taylor JW (2005) Cryptic speciation in the cosmopolitan and clonal human pathogenic fungus Aspergillus fumigatus. Evolution 59(9):18861899.

Quaedvlieg W, Kema GHJ, Groenewald JZ, Verkley GJM, Seifbarghi S, Razavi M, Mirzadi Gohari A, Mehrabi R, Crous PW (2011) Zymoseptoria gen. nov.: a new genus to accommodate Septoria-like species occurring on graminicolous hosts. Persoonia 26:57-69. doi: 10.3767/003158511X571841.

Rickerts V, Just-Nübling G, Konrad F, Kern J, Lambrecht E, Böhme A, Jacobi V, Bialek R (2006) Diagnosis of invasive aspergillosis and mucormycosis in immunocompromised patients by seminested PCR assay of tissue samples. Eur J Clin Microbiol Infect Dis. 25(1):8-13.

Rossetti F, Storti F, Salvini F (2000) Cenozoic noncoaxial transtension along the western shoulder of the Ross Sea, Antarctica, and the emplacement of McMurdo dyke arrays. Terra Nova 12:60-66. doi: 10.1111/j.1365-3121.2000.00270.x.

Ruibal C (2004) Isolation and characterization of melanized, slow-growing fungi from semiarid rock surfaces of central Spain and Mallorca. Doctoral dissertation. Universidad Autónoma de Madrid/Merck, Sharp \& Dohme de España, Madrid, Spain.

Ruibal C, Gonzalo P, Bills GF (2005) Isolation and characterization of melanized fungi from limestone formations in Mallorca. Mycol Prog 4:23-38. doi: 10.1007/s11557-006-0107-7 
Ruibal C, Platas G, Bills GF (2008) High diversity and morphological convergence among melanised fungi from rock formations in the Central Mountain System of Spain. Persoonia 21:93-110. doi: $10.3767 / 003158508 \times 371379$

Ruibal C, Gueidan C, Selbmann L, Gorbushina AA, Crous PW, Groenewald JZ, Muggia L, Grube M, Isola D, Schoch CL, Staley JT, Lutzoni F, de Hoog GS (2009) Phylogeny of rock-inhabiting fungi related to Dothideomycetes. Stud Mycol 64:123-133. doi:10.3114/sim.2009.64.06

Russell NJ (1998) Molecular adaptations in psychrophilic bacteria: potential for biotechnological applications. Adv. Biochem. Eng. Biotechnol. 61:1-21.

Samerpitak K, Van der Linde E, Choi HJ, Gerrits van den Ende AHG, Machouart M, Gueidan C, de Hoog GS (2013) Taxonomy of Ochroconis, a genus including opportunistic pathogens on humans and animals. Fung Div (in press). doi: 10.1007/s13225-013-0253-6

Schoch CL, Crous PW, Groenewald JZ, Boehm EWA, Burgess TI, De Gruyter J, de Hoog GS, Dixon LJ, Grube M, Gueidan C, Harada Y, Hatakeyama S, Hirayama K, Hosoya T, Huhndorf SM, Hyde KD, Jones EBG, Kohmeyer J, Kruys A, Li YM, Lücking R, Lumbush HT, Marvanová L, Mbatchou JS, McVay AH, Miller AN, Mugambi GK, Muggia L, Nelsen MP, Nelson P, Owensby CA, Phillips AJL, Phongpaichit S, Pointing SB, Pujade-Renaud V, Raja HA, Rivas Plata E, Robbertse B, Ruibal C, Sakayaroj J, Sano T, Selbmann L, Shearer CA, Shirouzu T, Slippers B, Suetrong S, Tanaka K, Volkmann- Kohlmeyer B, Wingfield MJ, Wood AR, Woudenberg JHC, Yonezawa H, Zhang Y, Spatafora JW (2009) A class-wide phylogenetic assessment of Dothideomycetes. Stud Mycol 64:1-15. doi: 10.3114/sim.2009.64.01

Shoch CL, Seifert KA, Huhndorf S, Robert V, Spouge JL, Levesque CA, Chen W, Fungal Barcoding Consortium (2012) Nuclear ribosomal internal transcribed spacer (ITS) region as a universal DNA barcode marker for Fungi. PNAS 109: 6241-6246. doi: 10.1073/pnas.1117018109.

Seifert KA, Nickerson NL, Corlett M, Jackson ED, Louis-Seize G, Davies RJ (2004) Devriesia, a new hyphomycete genus to accommodate heat-resistant, cladosporium-like fungi. Can J Bot 82:914926. doi: 10.1139/b04-070.

Selbmann L, de Hoog GS, Mazzaglia A, Friedmann EI, Onofri S (2005) Fungi at the edge of life: cryptoendolithic black fungi from Antarctic Desert. Stud Mycol 51:1-32

Selbmann L, de Hoog GS, Gerrits van den Ende AHG, Ruibal C, De Leo F, Zucconi L, Isola D, Ruisi S, Onofri S (2008) Drought meets acid: three new genera in a dothidealean clade of extremotolerant fungi. Stud Mycol 61:1-20. doi: 10.3114/sim.2008.61.01

Selbmann L, Isola D, Egidi E, Zucconi L, Gueidan C, de Hoog GS, Onofri S (2013a) Mountain tips as reservoirs for new rock fungal entities: Saxomyces gen. nov. and four new species from the Alps. Fung Div DOI: 10.1007/s13225-013-0234-9

Selbmann L, Grube M, Onofri S, Isola D, Zucconi L (2013b) Antarctic epilithic lichens as niches for meristematic fungi. Biology 2:784-797. doi:10.3390/bioology2020784

Selbmann L Hoog GS, Zucconi L, Isola D, Onofri S (2013c) Black yeasts from cold habitats. In: Seckbach J (ed) Yeasts from cold habitats. Springer, Berlin 
Sert HB, Sümbül H, Sterflinger K (2007a) Microcolonial fungi from antique marbles in Perge/Side/Termessos (Antalya/Turkey). Antonie Leeuwenhoek 91(3):217-227.

Sert HB, Sümbül H, Sterflinger K (2007b) Sarcinomyces sideticae, a new black yeast from historical marble monuments in Side (Antalya, Turkey). Bot J Linn Soc 154:373-380

Sert HB, Sümbül H, Sterflinger K (2007c) A new species of Capnobotryella from monument surfaces. Mycol Res 111:1235-1241

Sert HB, Sterflinger K (2010) A new Coniosporium species from historical marble monuments. Mycol Prog. 9(3): 353-359.

Skinner DNB (1980) GANOVEX '79. New Zealand Antarctic Record 3:15-24.

Smith ME, Douhan GW, Rizzo DM (2007) Ectomycorrhizal community structure in a xeric Quercus woodland based on rDNA sequence analysis of sporocarps and pooled roots. New Phytol 174:847863.

Spatafora JW, Mitchell TG, Vilgalys R (1995) Analysis of genes coding for small-subunit rRNA sequences in studying phylogenetics of dematiaceous fungal pathogens. Journal of Clinical Microbiology 33(5):1322-1326.

Spatafora JW, Sung GH, Johnson D, Hesse C, O’Rourke B, Serdani, M, Schoch CL (2006) A fivegene phylogeny of Pezizomycotina. Mycologia 98(6). 1018-1028.

Staley JT, Palmer F, Adams B (1982) Microcolonial fungi: common inhabitants on desert rocks? Science 215:1093-1095. doi: 10.1126/science.215.4536.1093.

Stamatakis A, Hoover P, Rougemont J (2008) A rapid bootstrap algorithm for the RAxML Web servers. Syst Biol; 57(5):758--771. doi: 10.1080/10635150802429642.

Sterflinger K, Krumbein WE (1997) Dematiaceous fungi as the main agent of biopitting on mediterranean marbles and limestones, Geomicrobiology Journal, 22, p.219-231.

Sterflinger K, de Baere R, de Hoog GS, de Wachter R, Krumbein WE, Haase G (1997) Coniosporium perforans and C. apollinis, two new rock-inhabiting fungi isolated from marble in the Sanctuary of Delos (Cyclades, Greece). Antonie van Leeuwenhoek 72:349-363. doi: 10.1023/A:1000570429688

Sterflinger K (1998) Temperature and NaCl-tolerance of rock-inhabiting meristematic fungi. Antonie van Leeuwenhoek 74:271-281. doi: 10.1023/A:1001753131034

Sterflinger K, de Hoog GS, Haase G (1999) Phylogeny and ecology of meristematic ascomycetes. Stud Mycol 43:5-22

Sterflinger K (2000) Fungi as geologic agents. Geomicrobiol J. 17(2): 97-124.

Sterflinger K, Prillinger H (2001) Molecular taxonomy and biodiversity of rock fungal communities in an urban environment (Vienna, Austria). Antonie Leeuwenhoek 80: 275-286 
Sterflinger K (2005) Black Yeasts and meristematic fungi: ecology, diversity and identification. In The Yeast handbook. Biodiversity and ecophysiology of yeasts; Seckbach, J. Eds. Springer-Verlag Berlin and Heidelberg GmbH \& Co. K. pp 501-514.

Sterflinger K (2006) Black yeast and meristematic fungi: ecology, diversity and identification. In: Péter G, Rosa C (eds) The yeast handbook. Biodiversity and ecophysiology of yeasts. Springer, Berlin, pp 501-514. doi: 10.1007/3-540-30985-3_20.

Sterflinger K, Tesei D, Zakharova K (2012) Fungi in hot and cold deserts with particular reference to microcolonial fungi. Fungi Ecology 5:453-462. doi:10.1016/j.funeco.2011.12.007.

Stukenbrock EH, Quaedvlieg W, Javan-Nikhah M, Zala M, Crous PW, McDonald BA (2012) Zymoseptoria ardabilia and Z. pseudotritici, two progenitor species of the Septoria tritici leaf blotch fungus Z. tritici (synonym: Mycosphaerella graminicola). Mycologia 104: 1397-1407. doi: 10.3852/11-374.

Taylor JW, Jacobson DJ, Kroken S, Kasuga T, Geiser DM, Hibbett DS, Fisher MC (2000) Phylogenetic species recognition and species concepts in fungi. Fungal genetics and biology 31(1): 21-32.

Tesei D, Marzban G, Zakharova K, Isola D, Selbmann L, Sterflinger K (2012) Alteration of protein patterns in black rock inhabiting fungi as a response to different temperatures. Fungal Biol 116:932940. doi:10.1016/j.funbio.2012.06.004.

Tsuneda A, Hambleton S, Curraha RS (2011) The anamorph genus Knufia and its phylogenetically allied species in Coniosporium, Sarcinomyces, and Phaeococcomyces. Botany 89(8): 523-536. doi: 10.1139/b11-041.

Van Uden N (1984) Temperature profiles of yeasts. Adv Microbiol Physiol 25:195-251.

Vaughan DG, Bamber JL, Giovinetto M, Russel J, Cooper APR (1999) Reassessment of net surface mass balance in Antarctica. J Climate 12:933-946.

Vilgalys R, Hester M (1990) Rapid genetic identification and mapping of enzymatically amplified ribosomal DNA from several Cryptococcus species. J Bacteriol 172:4238-4246.

Urzì C, Wollenzien U, Zagari M, Krumbein WE (1994) Biodiversity of the marble inhabiting microflora. Colonisation, biodeterioration and control. In: Proceedings of the $4^{\text {th }}$ Workshop, Eurocare - Euromarble, Aries, 1994. Bayerisches Landesamt fiir Denkmalpflege Zentrallabor, Forschungsber. 13 , pp $1-16$

Urzì C, Realini M (1998) Colours changes of Noto's calcareous sandstone as related to its colonization by microorganisms. J. Int. Biodet. Biodeg. 42: 45-54.

Warscheid J, Brahms J (200) Biodeterioration of stone: a review. J. Int. Biodet. Biodeg. 46:343-351.

Weinstein RN, Montiel PO, Johnstone K (2000) Influence of growth temperature on lipid and soluble carbohydrate synthesis by fungi isolated from fellfield soil in the maritime Antarctic. Mycologia 92:222-229. 
White TJ, Bruns T, Lee S, Taylor J (1990) Amplification and direct sequencing of fungal ribosomal RNA genes for phylogenetics. In PCR protocols, a guide to methods and applications. Innis M.A., Gelfe D.H., Sninsky J.J., White T.J. Eds. Academic Press, San Diego. pp. 315-322.

Weiß M, Selosse M-A, K.-H. Rexer K-H, Urban A, Oberwinkler F (2004) Sebacinales: A hitherto overlooked cosm of heterobasidiomycetes with a broad mycorrhizal potential. Mycological Research 108:1003-1010.

Wimpenny J (2000) An overview of biofilms as functional communities. In Community structure and co-operation in biofilms. Allison DG, Gilbert P, Lappin-Scott HM, and Wilson M. Eds., Cambridge University Press, Cambridge, UK pp. 1-24.

Wingfield MJ, Slippers B, Roux J, Wingfield BD (2001) Worldwide Movement of Exotic Forest Fungi, Especially in the Tropics and the Southern Hemisphere. BioScience 51(2):134-140.

Whittaker RH (1970) Communities And ecosystems. Macrnillan, New York. Pp 162.

Wynn-Williams DD, Edwards HGM, Garcia-Pichel F (1999) Functional biomolecules of Antarctic stromatolitic and endolithic cyanobacterial communities. Eur. J. Phycol. 34:381-391.

Wollenzien U, de Hoog GS, Krumbein WE, Urzì C (1995) On the isolation of microcolonial fungi occurring on and in marble and other calcareous rocks. Sci Total Environ 167:287-294. doi: 10.1016/0048-9697(95)04589-S

Wollenzien U, de Hoog GS, Krumbein WE, Uijthof JMJ. (1997) Sarcinomyces petricola, a new microcolonial fungus from marble in the Mediterranean basin. Antonie Leeuwenhoek 71:281-288.

Wubet T, Weiss M, Kottke I, Teketay D, Oberwinkler F (2004) Molecular diversity of arbuscular mycorrhizal fungi in Prunus africana, an endangered medicinal tree species in dry Afromontane forests of Ethiopia. New Phytologist 161:517-528.

Yoshida S, Takeo K, Hoog GS de, Nishimura K, Miyaji M (1996) A new type of growth exhibited by Trimmatostroma abietis. Antonie van Leeuwenhoek 69:211-215.

Zalar P, de Hoog GS, Gunde-Cimerman N (1999) Ecology of halotolerant dothideaceous black yeasts. Stud Mycol 43:38-48.

Zhdanova NN, Zakharchenko VA, Vember VV, Nakonechnaya LT (2000) Fungi from Chernobyl: mycobiota of the inner regions of the containment structures of the damaged nuclear reactor. Mycol Res 104:1421-1426.

Zakharova K, Tesei D, Marzban G, Dijksterhuis J, Wyatt T, Sterflinger K (2012) Microcolonial fungi on rocks: a life in constant drought? Mycopathologia 175:537-547. doi: 10.1007/s11046-0129592-1

Zucconi L, Gagliardi M, Isola D, Onofri S, Andaloro MC, Pelosi C, Pogliani P, Selbmann L (2012) Biodeterioration agents dwelling in or on the wall paintings of the Holy Saviour's cave (Vallerano, Italy) . Int Biodet Biodeg 70:40-46. doi: 10.1016/j.ibiod.2011.11.018 


\section{ANNEXES}

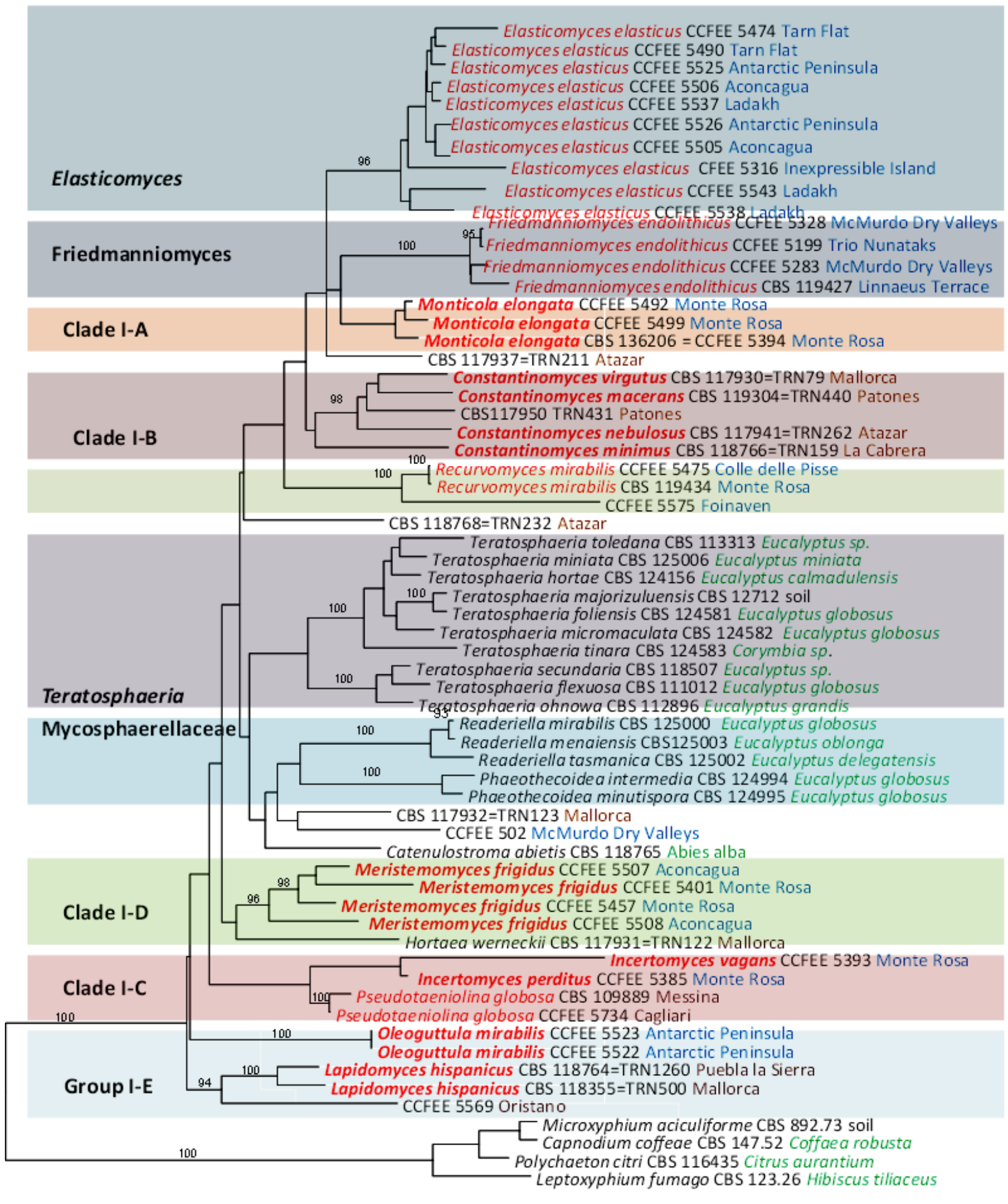

Fig A. Four-gene phylogenetic tree of family Teratosphaeriaceae I, resulting from a RAxML analysis of the nucLSU, RPB2, ITS, BT2 sequence alignment. Boostrap values of $\geq 95 \%$ are provided above corresponding nodes and in a thicker line. Clades and groups are indicated with colored blockswith the isolation source or locality of origin: in blue text are mountain peaks and cold environments, in brown are Mediterranean localities and in green are plant origins. Strains isolated from rocks are in red text and species described here for the first time in bold 


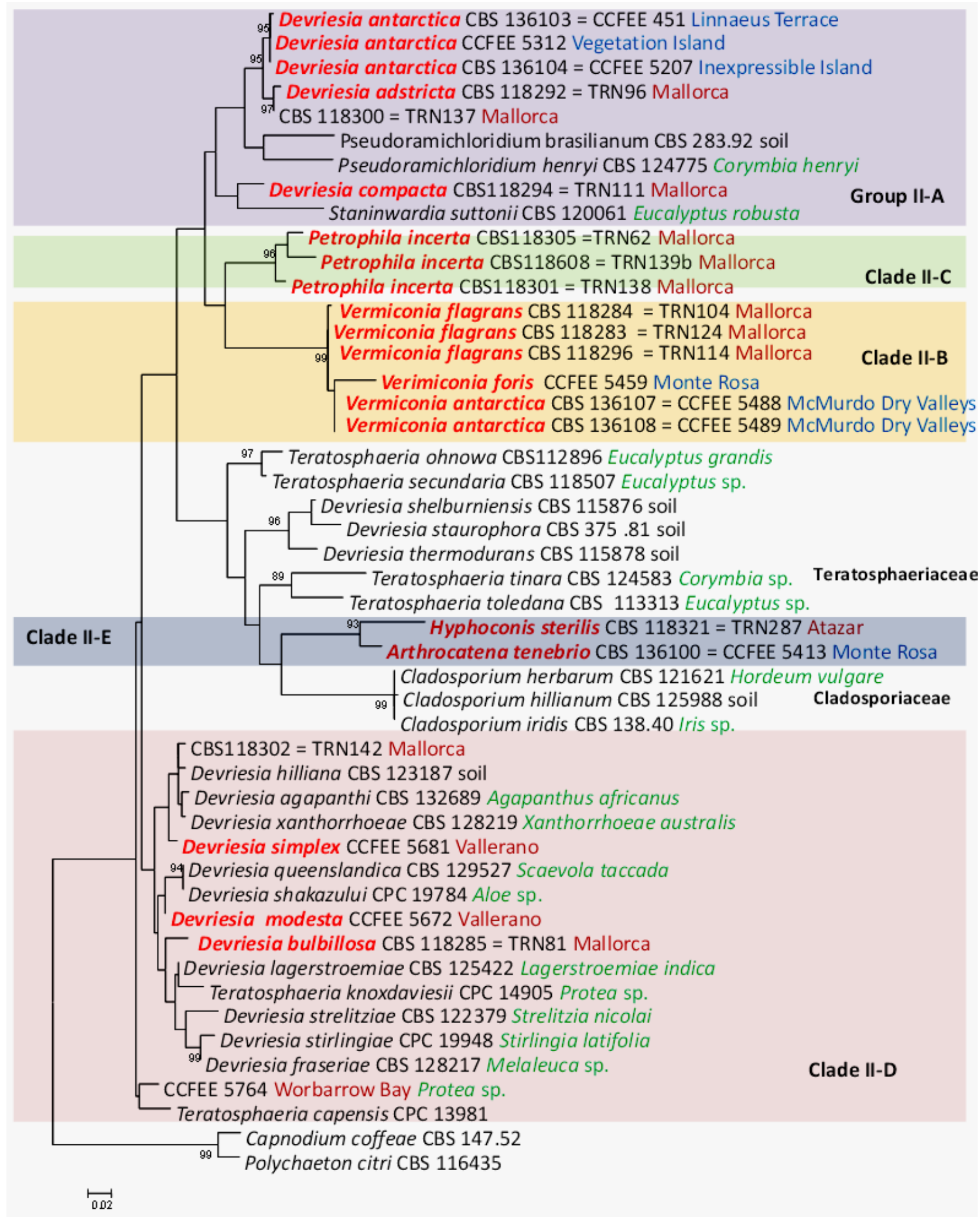

Fig B. Four-gene phylogenetic tree of family Teratospaheriaceae II, resulting from a RAxML analysis of the nucLSU, RPB2, ITS, BT2 sequence alignment. Boostrap values of $\geq 95 \%$ are provided above corresponding nodes and in a thicker line. Clades and groups are indicated with colored blockswith the isolation source or locality of origin: in blue text are mountain peaks and cold environments, in brown are Mediterranean localities and in green are plant origins. Strains isolated from rocks are in red text and species described here for the first time in bold 


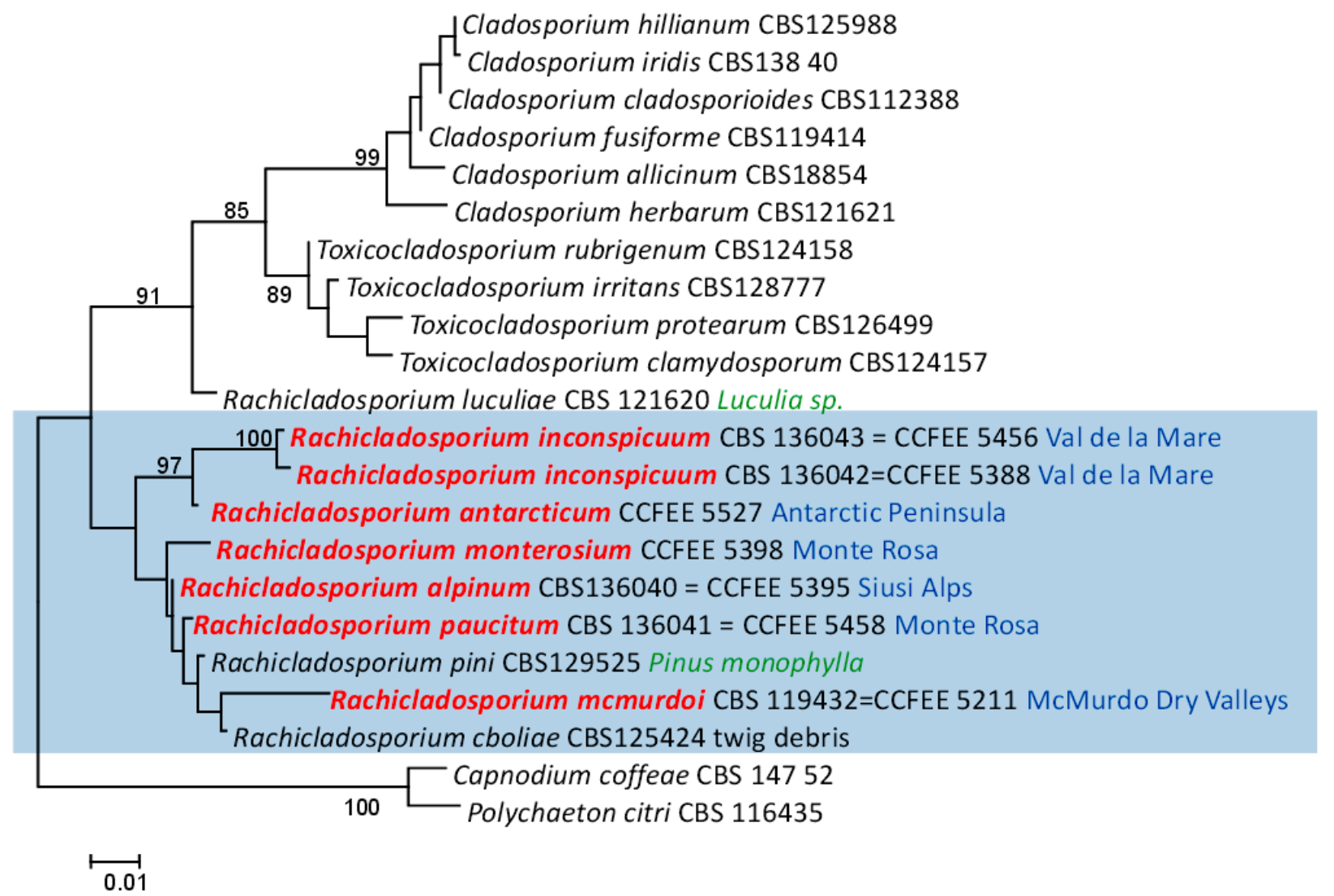

Fig C. Four-gene phylogenetic tree of family Cladosporiaceae, resulting from a RAxML analysis of the nucLSU, RPB2, ITS, BT2 sequence alignment. Boostrap values of $\geq 95 \%$ are provided above corresponding nodes and in a thicker line. Clades and groups are indicated with colored blockswith the isolation source or locality of origin: in blue text are mountain peaks and cold environments, in brown are Mediterranean localities and in green are plant origins. Strains isolated from rocks are in red text and species described here for the first time in bold 
